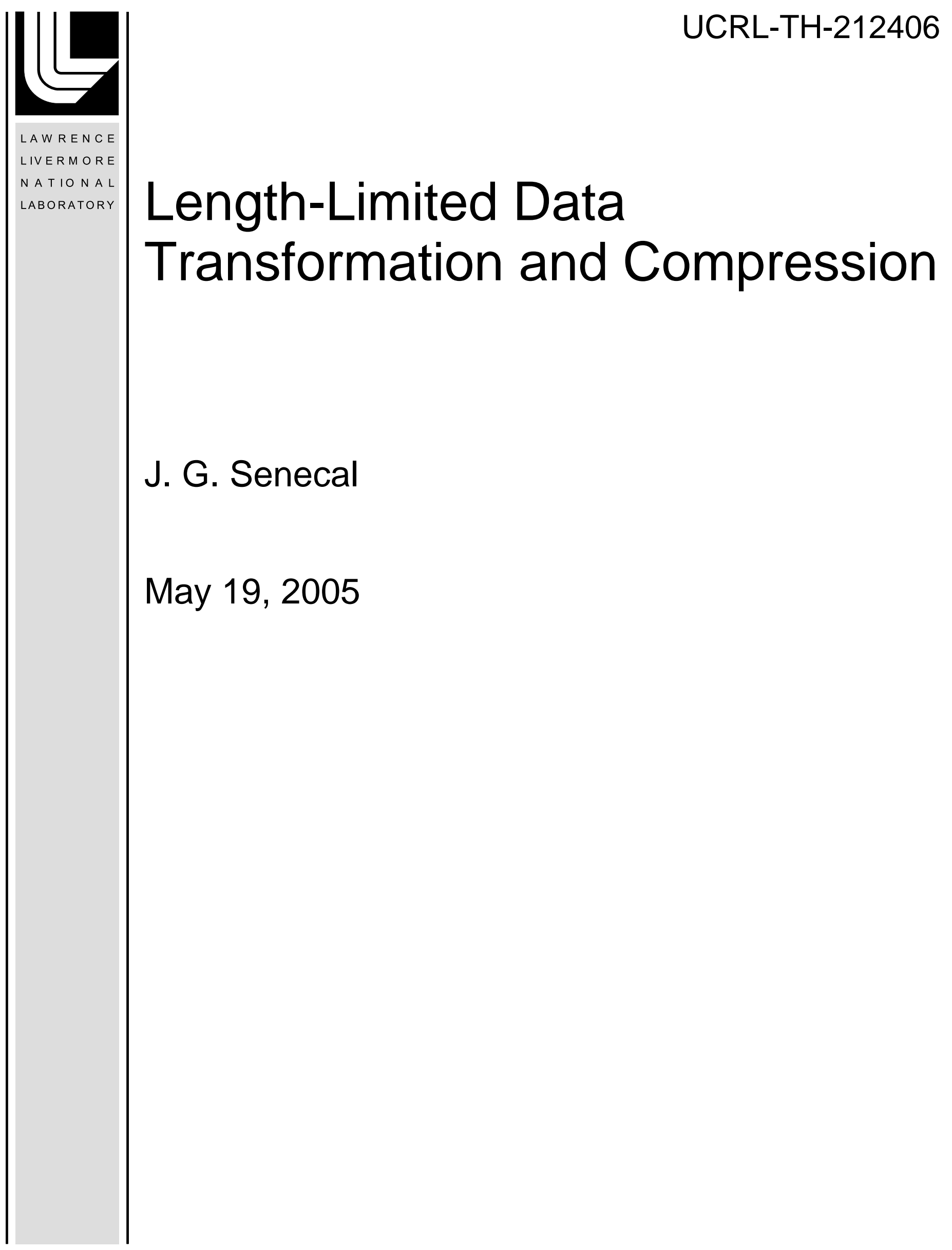


This document was prepared as an account of work sponsored by an agency of the United States Government. Neither the United States Government nor the University of California nor any of their employees, makes any warranty, express or implied, or assumes any legal liability or responsibility for the accuracy, completeness, or usefulness of any information, apparatus, product, or process disclosed, or represents that its use would not infringe privately owned rights. Reference herein to any specific commercial product, process, or service by trade name, trademark, manufacturer, or otherwise, does not necessarily constitute or imply its endorsement, recommendation, or favoring by the United States Government or the University of California. The views and opinions of authors expressed herein do not necessarily state or reflect those of the United States Government or the University of California, and shall not be used for advertising or product endorsement purposes.

This work was performed under the auspices of the U.S. Department of Energy by University of California, Lawrence Livermore National Laboratory under Contract W-7405-Eng-48. 
Joshua G. Senecal

September 2005

Computer Science

Length-limited Data Transformation and Compression

\begin{abstract}
$\underline{\text { Abstract }}$
Scientific computation is used for the simulation of increasingly complex phenomena, and generates data sets of ever increasing size, often on the order of terabytes. All of this data creates difficulties. Several problems that have been identified are (1) the inability to effectively handle the massive amounts of data created, (2) the inability to get the data off the computer and into storage fast enough, and (3) the inability of a remote user to easily obtain a rendered image of the data resulting from a simulation run. This dissertation presents several techniques that were developed to address these issues. The first is a prototype bin coder based on variable-tovariable length codes. The codes utilized are created through a process of parse tree leaf merging, rather than the common practice of leaf extension. This coder is very fast and its compression efficiency is comparable to other state-of-the-art coders. The second contribution is the Piecewise-Linear Haar (PLHaar) transform, a reversible $n$-bit to $n$-bit wavelet-like transform. PLHaar is simple to implement, ideal for environments where transform coefficients must be kept the same size as the original data, and is the only $n$-bit to $n$-bit transform suitable for both lossy and lossless coding.
\end{abstract}




\title{
Length-limited Data Transformation and Compression
}

\author{
By \\ JOSHUA G. SENECAL \\ B.S., University of California, San Diego, 1999 \\ DISSERTATION
}

Submitted in partial satisfaction of the requirements for the degree of DOCTOR OF PHILOSOPHY

in

Computer Science

in the

OFFICE OF GRADUATE STUDIES

of the

UNIVERSITY OF CALIFORNIA

DAVIS

Approved:

Committee in charge 


\title{
Length-limited Data Transformation and Compression
}

\author{
Copyright 2005 \\ by \\ Joshua G. Senecal
}


First, I dedicate this work to my family. My dear wife, Young-Oh, has been a constant support to me in all my studies, and has always believed in me. I can honestly say that without her I never would have made it this far. Our children, Neil and Edwin, have also given me much-needed comfort and distraction, and have enabled me to keep the correct perspective through all my academic endeavors.

Second, I dedicate this to my mother, who placed a high value on a good education and encouraged me to excel. Also Dr. Bimal Kad, from the Department of Structural Engineering at UC San Diego, who mentored me in my undergraduate research. It was under his guidance that I had my first experiences in researching, publishing, and presenting. While working with him I developed an interest in research and a desire to further my education by attending graduate school. All through the process he was patient and helpful.

Last of all I must thank my Father in Heaven, for giving me such a great opportunity and surrounding me with the people who would help me make it happen. 


\section{Acknowledgments}

One of the lasting things that I learned while earning my Ph.D. is that an undertaking of this size is not something solely mine. There are many people (in particular, my collaborators) who contributed ideas, support, and counsel. All of these things were necessary to create this finished product. My use of the terms "we", "our", etc., in this work is in recognition of their contributions.

In the three years or so that I worked at Lawrence Livermore National Laboratory I was supervised and guided by Dr. Mark Duchaineau. Working with Mark has been great; he is full of ideas, enthusiasm, and (especially) patience.

Professor Ken Joy at UC Davis is a wonderful mentor, and was always willing to spend time discussing an issue, providing good insights and counsel. Like Mark, he is patient, enthusiastic about his work and students, and is full of ideas.

While looking at some of the work that Mark and I did regarding $n$-bit to $n$-bit transforms, Peter Lindstrom came up with the idea behind the Piecewise-Linear Haar transform. I did a lot of the work investigating and evaluating it, but the original idea was his.

I collaborated with Ben Gregorski on his research on extracting isosurfaces from time-varying volume data. Some of the material from chapter 5, primarily the figures, tables, and equations, was provided by him.

The students in the Institute for Data Analysis and Visualization at UC Davis, as well as the people in our research group at Lawrence Livermore National Laboratory, are a great group of people to work with. All of them have always been helpful, positive, friendly, and patient. Their willingness to discuss an idea, work out a problem, and tell me what they think, frankly but in a constructive fashion, allowed me to greatly improve the quality of my work. I appreciate their contributions.

The quality of this work was improved by the anonymous reviewers who reviewed the publications resulting from my research. Also, I thank Henrique Malvar at Microsoft Research, and Aaron Kiely and Matthew Klimesh at NASA's Jet Propulsion Laboratory for their comments and suggestions. Peter Stubley, whose doctoral dissertation dealt with 
variable-to-variable length codes, provided valuable assistance and advice. Leon Bottou and William Douglas Withers provided valuable assistance with the Z-Coder and ELSCoder, respectively. Andrew Turpin provided valuable assistance with the Package-Merge algorithm, as well as source code that was used in my work. My brother Matthew reviewed the next-to-final draft of this dissertation and provided excellent suggestions.

Demonstrating data transformation techniques on images requires images. I compiled a suite of various images from a variety of sources. Images of black-and-white lines, and computer-rendered objects were provided by Michael J. Tarr at Brown University (Providence, RI)[52]. Some photographic images were obtained from The Signal and Image Processing Institute at the University of Southern California[1] and The Waterloo Bragzone[5]. Some of the line art images were made available for download by Apple Computer, Inc. through its software AppleWorks. Fingerprint images (see figure 7.5) were obtained from the Fingerprint Verification Competition 2000 website [7]. CCITT facsimile test images were obtained from the Finnish Academic and Research Network anonymous FTP server [53]. MRI data was obtained from The Department of Radiology at UCSD Medical Center and the Vision List Imagery Archive [4]. My mother, Robin Senecal-Hicks gave permission for her MRI to be released. I am grateful to all of these people and organizations for making images available for use.

The wavelet decomposition images in figure 6.2 are from the 1996 SIGGRAPH course "Wavelets in Computer Graphics", edited by Wim Sweldens and Peter Schröder, and were provided by them.

The bulk of this work was done while I was a research fellow at Lawrence Livermore National Laboratory, under the Student-Employee Graduate Research Fellowship program. I am grateful to those who provide and supervise this program.

This work was performed under the auspices of the U.S. Department of Energy by University of California Lawrence Livermore National Laboratory under contract No. W-7405-Eng-48.

This work was supported in part by a United States Department of Education Government Assistance in Areas of National Need (DOE-GAANN) grant \#P200A980307. 


\section{Contents}

List of Figures viii

List of Tables $\quad$ xii

Abstract $\quad$ xiv

1 Overview 1

1.1 Lots of Computers, Lots of Data . . . . . . . . . . . . . . . 1

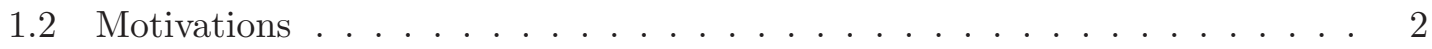

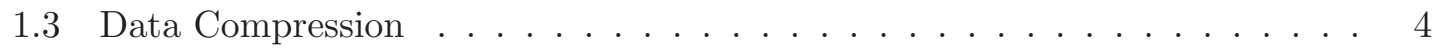

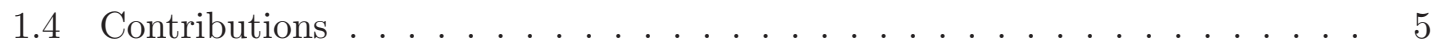

1.4.1 Length-limited Variable-to-Variable Length Codes . . . . . . . . . . 5

$1.4 .2 n$-bit to $n$-bit Transforms . . . . . . . . . . . . . 5

I Coding 6

2 Information Theory 7

2.1 Messages, Information, and Prediction . . . . . . . . . . . . 7

2.2 Entropy of a Memoryless Source . . . . . . . . . . . . . . . . . . 8

2.3 Conditional Entropy . . . . . . . . . . . . . . . . . . . 11

3 Data Compression $\quad 13$

3.1 Introductory Background . . . . . . . . . . . . . . . . . 13

3.1 .1 Coding Inefficiency . . . . . . . . . . . . . . . . . 14

3.1 .2 Lossy Coding . . . . . . . . . . . . . . . . . . . 15

3.2 Some Data Compression Techniques . . . . . . . . . . . . . . 18

3.2.1 Run-Length Encoding . . . . . . . . . . . . . . . 18

3.2 .2 Golomb Coding . . . . . . . . . . . . . . . . . . 19

3.2 .3 Huffman Codes . . . . . . . . . . . . . . . . . . . . . 20

3.2.4 Parse Tree Methods . . . . . . . . . . . . . . . . . 22

3.2.5 Arithmetic Codes . . . . . . . . . . . . . . . . . 25

4 A High-performance Encoder Using Variable-to-variable Length Codes 26

4.1 Introduction . . . . . . . . . . . . . . . . . . . . . . . . . . . . . . 6

4.2 Encoding Using Table-lookups . . . . . . . . . . . . . . . 27 
4.2 .1 A Simple, Fast Process . . . . . . . . . . . . . . . . . . 27

4.2 .2 Limiting Table Size . . . . . . . . . . . . . . . . . . 27

4.3 Generating a Length-limited VV Code . . . . . . . . . . . . . . . 30

4.3 .1 Extension . . . . . . . . . . . . . . . 30

4.3 .2 Source String Merging . . . . . . . . . . . . . . . . . . 32

4.4 Properties of the Greedy Merging Process . . . . . . . . . . . . . . . . 33

4.5 Properties of Merged VV Codes . . . . . . . . . . . . . . . . 37

4.5.1 Using VV Codes in Data Compression . . . . . . . . . . . . . . . . . . 43

4.6 Bin Coding . . . . . . . . . . . . . . . . . . . 43

4.6 .1 Bin Output Interleaving . . . . . . . . . . . . . . . 44

4.7 A Hybrid Bin Coder Utilizing VV Codes . . . . . . . . . . . . . . . . . . . 45

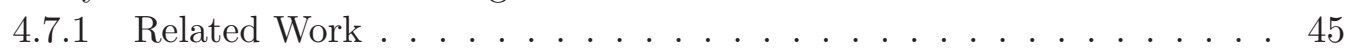

4.7 .2 Selecting VV Codes . . . . . . . . . . . . . . . . 46

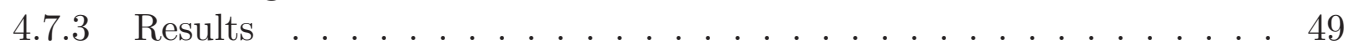

4.8 Discussion . . . . . . . . . . . . . . . . . . . . . 53

4.9 Future Work . . . . . . . . . . . . . . . . . . 53

5 Lead-1 Encoding and Surface Contouring $\quad 55$

5.1 Volumes and Isosurfaces . . . . . . . . . . . . . . . . . 55

5.2 Time-varying Data and Contouring . . . . . . . . . . 56

5.3 Lead-1 Encoding . . . . . . . . . . . . . . . . . . . . 57

5.4 Results . . . . . . . . . . . . . . . . . . . . 59

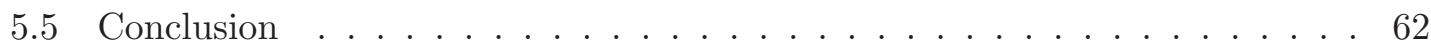

II Data Transformation $\quad 63$

6 Wavelets $\quad 64$

6.1 Introduction . . . . . . . . . . . . . . . . . . . 64

6.2 The Haar Wavelet Transform . . . . . . . . . . . . . . . . . . . . 65

6.3 Advantages of Wavelets . . . . . . . . . . . . . . . . . . 65

6.4 Dynamic Range Expansion . . . . . . . . . . . . . . . . . . . 70

6.5 Removing DRE: The S-Transform . . . . . . . . . . . . . . . . 72

$7 \quad N$-bit to $N$-bit Transforms $\quad \mathbf{7 4}$

7.1 More on Dynamic Range Expansion . . . . . . . . . . . . . . . . . . . 74

7.1.1 Can Dynamic Range Expansion be Eliminated? . . . . . . . . . . . . 75

7.1.2 Review: The Method of Chao, Fisher, and Hua . . . . . . . . . . . 75

7.2 Transform Evaluation . . . . . . . . . . . . . . . . 78

7.2.1 Peak Signal-to-Noise Ratio . . . . . . . . . . . . . . . . 78

$7.2 .2 L_{\infty}$ Error . . . . . . . . . . . . . . . . . . . 80

7.2 .3 Images . . . . . . . . . . . . . . . . . . . 80

8 Table-Lookup Haar $\quad 83$

8.1 Transform LUTs . . . . . . . . . . . . . . . . . . . . . . 84

8.1 .1 Discontinuities . . . . . . . . . . . . . . . 87

8.2 Sorted LUTs Are not Unique . . . . . . . . . . . . . . . . . . 88

8.3 TLHaar Implementation Optimizations . . . . . . . . . . . . . . 88 
8.4 Results . . . . . . . . . . . . . . . . . . . . 90

8.4.1 Execution Time . . . . . . . . . . . . . . . . . . 90

8.4 .2 Compressibility of Coefficients . . . . . . . . . . . . 90

8.5 Conclusions and Possible Extensions . . . . . . . . . . . . . . . . 93

9 Piecewise-Linear Haar $\quad 95$

9.1 Transformation by Rotation . . . . . . . . . . . . . . . . . 95

9.1.1 Haar: an $L_{2}$ Rotation . . . . . . . . . . . . . . . . . . . 95

9.1.2 PLHaar: an $L_{\infty}$ Rotation . . . . . . . . . . . . . . . . . 96

9.2 Efficiency Considerations . . . . . . . . . . . . . . . . . . . 99

9.3 Characteristics of PLHaar . . . . . . . . . . . . . . . . . . . . 99

9.3.1 Data Decorrelation . . . . . . . . . . . . . . . . . 99

9.3.2 No Average Preservation . . . . . . . . . . . . . . . . . . . 102

9.3 .3 Signed Zeros . . . . . . . . . . . . . . . . . . . . . . . . 103

9.4 Implementation . . . . . . . . . . . . . . . . . . . . . . . . . 104

9.5 Results . . . . . . . . . . . . . . . . . . 105

9.5.1 Execution Time. . . . . . . . . . . . . . . . 105

9.5.2 Quantization and PSNR . . . . . . . . . . . . 107

9.5.3 Thresholding and PSNR . . . . . . . . . . . . . 112

9.6 Signed Zeros in PLHaar2 f . . . . . . . . . . . . . . . . . 116

9.6.1 Predicting Signed Zeros . . . . . . . . . . . . . . . 116

9.6.2 Signed Zeros and PSNR . . . . . . . . . . . . . . . 117

9.7 Conclusions and Possible Extensions . . . . . . . . . . . . . 120

10 Conclusions and Future Directions $\quad 121$

$\begin{array}{lr}\text { Bibliography } & 123\end{array}$ 


\section{List of Figures}

1.1 Static data compression process. . . . . . . . . . . . . . . . 4

1.2 Adaptive data compression process. . . . . . . . . . . . 5

2.1 Binary entropy curve. . . . . . . . . . . . . . . . 9

3.1 Sample inefficiency curve for a code that is most efficient at $q=0.42 \ldots \ldots 16$

3.2 Inefficiency curves for three codes. . . . . . . . . . . . . . . 16

3.3 Inefficiency curve for a coder using the three codes from figure $3.2 . \quad \ldots . .17$

3.4 An example of lossy coding with JPEG. The image on the left has been coded with little loss, the image on the right with extensive loss. . . . . . . 17

3.5 Inefficiency curve for Golomb codes. Note the great inefficiency at about

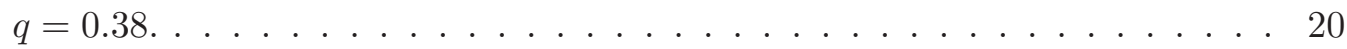

3.6 An illustration of the Huffman code generation process. A left branch is a

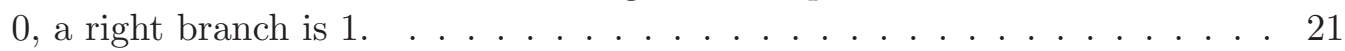

3.7 A BV code parse tree and code table. $B=3$, and codes are generated using the Huffman algorithm. . . . . . . . . . . . . . . . . . . 22

3.8 The creation of a VB parse tree, by extending and splitting the most probable leaf. . . . . . . . . . . . . . . . . . . . . 24

3.9 A VV parse tree (created using the Tunstall algorithm) and its code table. Codes are generated using the Huffman algorithm. . . . . . . . . . . . . . 24

4.1 Encoding via table-lookup. . . . . . . . . . . . . . . . . . 28

4.2 Coding inefficiency of length-limited codes. . . . . . . . . . . . . 31

4.3 Coding inefficiency of some VV codes. . . . . . . . . . . . . . . 31

4.4 Source String Merging. The underlined numbers indicate the code lengths. 34

4.5 Parse tree created when $q=0.40$, parse tree strings are limited to 10 bits

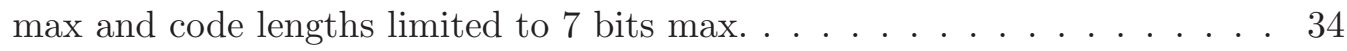

4.6 Illustration of proof of why the greedy merging process always selects a leaf that is part of a pair (i.e. has a sibling). A 0 corresponds to a left branch. . 35

4.7 Illustration of proof of why the greedy merging process always causes the smallest decrease in the parse tree's total weighted path length. In this example $p=0.8, q=0.2$. A 0 corresponds to a left branch. . . . . . . 36

4.8 Illustration of how the greedy merging process does not always result in the smallest coding rate. Results for a greedy merge. In this example $p=0.6$, $q=0.4$. A 0 corresponds to a left branch. . . . . . . . . . . 38 
4.9 Illustration of how the greedy merging process does not always result in the smallest coding rate. Results for an alternative merge. In this example $p=0.6, q=0.4$. A 0 corresponds to a left branch. . . . . . . . . . 39

4.10 Coding Rate when $q=0.10 \ldots \ldots \ldots \ldots \ldots$. . . . . . . . . 41

4.11 Coding Rate when $q=0.20 \ldots \ldots \ldots \ldots$. . . . . . . . . . . 41

4.12 Coding Rate when $q=0.35 \ldots \ldots \ldots \ldots$. . . . . . . . . . . 42

4.13 Coding Rate when $q=0.45 \ldots \ldots \ldots \ldots \ldots$. . . . . . . . . . . . . . . . . . . . . . .

4.14 Diagram of a simple bin coder. . . . . . . . . . . . . . . 44

4.15 Inefficiency curve of the best merged VV codes. . . . . . . . . . . . . . 47

4.16 Some test probability distribution curves, computed according to equation 4.5. The number by each curve is $x$, and the bit position is $i$. . . . . . . 50

4.17 Execution time of the coders. . . . . . . . . . . . . . . 51

4.18 Coding Inefficiency of the five coders. . . . . . . . . . . . . 51

4.19 Coding Inefficiency vs. Time of the 22 -bin hybrid bin coder. . . . . . . . . 52

4.20 Coding Inefficiency vs. Time of the 25-bin hybrid bin coder. . . . . . . . . 52

5.1 Two example isosurfaces. On the left is a surface of the engine block, on the right a surface of some Carbon "buckyball" molecules. . . . . . . . . . 56

5.2 Histogram of coefficient magnitudes in a $256^{3}$ volume with 274 time steps. The vertical axis is on a logarithmic scale. . . . . . . . . . . . . . 58

5.3 Page faults, in disk read sizes of (left to right) 10, 15, and 20 uncompressed time steps. . . . . . . . . . . . . . . . . . . 6 60

5.4 Frame rate, in disk read sizes of (left to right) 10, 15, and 20 uncompressed time steps. . . . . . . . . . . . . . . . . . . 6 61

6.1 Haar Wavelet Decomposition. . . . . . . . . . . . . . . . . 66

6.2 Haar Wavelet decomposition of an image. Images are from the 1996 SIGGRAPH course "Wavelets in Computer Graphics", edited by Wim Sweldens and Peter Schröder. . . . . . . . . . . . . . . . . 67

6.3 Histogram of Lena image (top), and histogram of transform coefficients after image decomposition by a wavelet transform. . . . . . . . . . . . 68

6.4 Dynamic Range Expansion in the Haar transform. . . . . . . . . . . . . . . 71

6.5 The non-normalized Haar transform. . . . . . . . . . . . . . . . 72

6.6 Eliminating low-pass dynamic range expansion by "squashing". . . . . . . . 73

7.1 CFH transform lookup tables. The forward transform is on the left, and the inverse is on the right. . . . . . . . . . . . . . . 77

7.2 A diagram of the inverse $\mathrm{CFH}$ transform table, showing the axes and discontinuities. If a point $(L, H)$ is located near one of the discontinuities, a process such as quantization will pull $(L, H)$ across the discontinuity to a new point $\left(L^{\prime}, H^{\prime}\right)$. Artifacts will result upon reconstruction. . . . . . . . . 77

7.3 The Wedding photo, transformed by $\mathrm{CFH}$ and thresholded to an equivalent entropy of one bit per pixel. . . . . . . . . . . . . . . . . . . 78

7.4 One of the dangers of relying on computed metrics to determine image quality. The PSNRs of both reconstructions are almost identical, but Reconstruction 2 is obviously a much better reconstruction. . . . . . . . . . . 80

7.5 Test images. From top, left to right: MRI, Fingerprint, ObjectBank, Photos, LineArt, CCITT. 
8.1 Forward and inverse transforms resulting from the sort. $A$ and $L$ are on the horizontal axes, and $B$ and $H$ on the vertical. The origin $(0,0)$ is the lower-left corner of each table. Black indicates a value of 0 , and white a

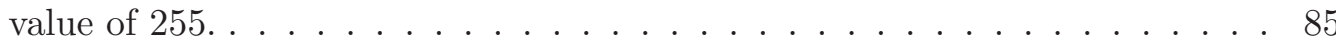

8.2 LH2AB LUT for $n=3$, showing a column and row breakout. Entries are

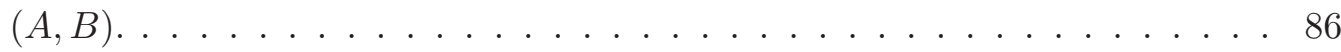

8.3 Histogram of the "Lena" image, before and after transformation. . . . . . . 86

8.4 TLHaar's discontinuities, and their effects. The Mandrill reconstruction is after quantization to 6 bits. . . . . . . . . . . . . . . . 87

8.5 Transform tables resulting from a randomized initialization. . . . . . . . . 89

8.6 TLHaar row transform data reordering optimization. The top shows the normal transform procedure, and the bottom the reordering procedure. . . 90

8.7 LUT access images (log normalized) for four types of images. Lighter pixels indicate more accesses to that entry. . . . . . . . . . . . . . . 94

9.1 Haar $L_{2}$ rotation. . . . . . . . . . . . . . . . . . . . . . . 96

9.2 PLHaar $L_{\infty}$ rotation. . . . . . . . . . . . . . . . . . . . 97

9.3 The transform LUTs for (clockwise from the upper-left) S-Transform, CFH, PLHaar, and TLHaar. Here we use $A-B$ (and not $B-A$ ) when computing $H$. The PLHaar table is the only one that does not have discontinuities or out-of-range values. . . . . . . . . . . . . . . . 100

9.4 PLHaar interpreted as an involution. Note that both rotations are in the same direction. . . . . . . . . . . . . . . . . . . . . 100

9.5 Zero-order entropy of the wavelet coefficients produced by the transforms. . 101

9.6 Histograms of "Lena" image, and the S-Transform, PLHaar, TLHaar, and CFH coefficients. . . . . . . . . . . . . . . . . 102

9.7 Source code for the continuous PLHaar transform. . . . . . . . . . . . . . 105

9.8 Source code for the discrete PLHaar transform . . . . . . . . . . . . . . . 106

9.9 Times for direct computation. . . . . . . . . . . . . . . . . 108

9.10 Times for table-lookup. . . . . . . . . . . . . . . . . 108

9.11 Bike: PSNR values for increasing quantization precision. For the $n$-bit to $n$-bit transforms the PSNR values for 8 bits (or no) quantization are not shown, as in this case the PSNR is infinity. . . . . . . . . . . . . 110

9.12 Bike: $L_{\infty}$ error values for increasing quantization precision. . . . . . . . . 110

9.13 Bike [6] image and details, transformed by CFH (left) and PLHaar (right) and quantized to 3 bits. . . . . . . . . . . . . . . . . 111

9.14 Barbara transformed by $\mathrm{CFH}$ (left) and PLHaar (right), and quantized to 3 bits. . . . . . . . . . . . . . . . . . . . . 112

9.15 Lena, quantized to five, four, and three bits. From the top: TLHaar, CFH, PLHaar, and S-Transform. . . . . . . . . . . . . . . . . 113

9.16 Mandrill, thresholded to an approximate entropy of (from left) four, two, and 0.5 bits per pixel. Upper images are CFH, lower are PLHaar1. PSNR is given beneath each image. . . . . . . . . . . . . . . . . . . . 114

9.17 Wedding photo, thresholded to an approximate entropy of (from left) 2.1, 1, and 0.5 bits per pixel. Upper images are CFH, lower are PLHaar2. PSNR is given beneath each image. . . . . . . . . . . . . . . . . 115

9.18 PSNR vs. Entropy graphs for four test images. . . . . . . . . . . 116 
9.19 Amount of zero sign correlation for PC and HL coefficient relationships. A value of 50 indicates no correlation, and 100 indicates perfect correlation. . 118

9.20 PSNR vs. Entropy graphs for four test images using PLHaar2. . . . . . . . 119 


\section{List of Tables}

3.1 A comparison of RLE and Golomb codes for $m=14$. The Golomb code uses less bits. . . . . . . . . . . . . . . . . . . . 19

4.1 Bins for the 22 -bin coder. . . . . . . . . . . . . . . . . 48

5.1 Lead-1 position counts for a $256^{3} \times 274$ dataset. . . . . . . . . . . . . 59

8.1 Test image categories and transformation times. (reord) indicates execution time using the reordering method of section 8.3. . . . . . . . . . . . 91

8.2 Compressed category sizes (in bytes) . . . . . . . . . . . . . . . 92 


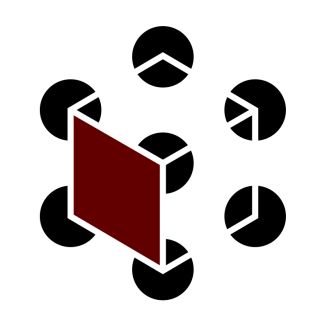
Length-limited Data Transformation
and Compression

\author{
Author: Joshua G. Senecal \\ Committee: Professor. Kenneth I. Joy, Chair \\ Professor. Bernd Hamann \\ Dr. Mark A. Duchaineau
}

Visualization and Graphics Research Group Institute for Data ANALysis and Visualization

Department of Computer Science

University of CALIFornia, Davis 


\begin{abstract}
Scientific computation is used for the simulation of increasingly complex phenomena, and generates data sets of ever increasing size, often on the order of terabytes. All of this data creates difficulties. Several problems that have been identified are (1) the inability to effectively handle the massive amounts of data created, $(2)$ the inability to get the data off the computer and into storage fast enough, and (3) the inability of a remote user to easily obtain a rendered image of the data resulting from a simulation run. This dissertation presents several techniques that were developed to address these issues. The first is a prototype bin coder based on variable-to-variable length codes. The codes utilized are created through a process of parse tree leaf merging, rather than the common practice of leaf extension. This coder is very fast and its compression efficiency is comparable to other state-of-the-art coders. The second contribution is the Piecewise-Linear Haar (PLHaar) transform, a reversible $n$-bit to $n$-bit wavelet-like transform. PLHaar is simple to implement, ideal for environments where transform coefficients must be kept the same size as the original data, and is the only $n$-bit to $n$-bit transform suitable for both lossy and lossless coding.
\end{abstract}




\section{Chapter 1}

\section{Overview}

\subsection{Lots of Computers, Lots of Data}

The past decade has seen an explosion in the number of computers and other digital devices, and the number of people who use them. Computers, digital music players, digital cameras, digital home theaters, and the like are becoming more prevalent and (most importantly) cheaper. This, coupled with the phenomenal growth of the Internet, means that there is an increasing number of digital files being created, stored, and transmitted. Graphics cards, used for quickly rendering high-quality images of three-dimensional scenes, are now tremendously powerful, exceeding traditional processors at floating-point operations per second. On the academic front, as computer parts become more powerful and fall in cost, supercomputing is coming within the reach of more and more organizations. Many supercomputers are clusters of commodity, off-the-shelf components. Some notable examples are Virginia Tech's System X supercomputer [3], ranked 7th most powerful computer in the world as of January 2005. The original supercomputer, a cluster of Apple PowerMac G5s (dual), went from conception to completion in 90 days. The FlashMob Supercomputing project [2] at the University of San Francisco, which attempts to create a powerful supercomputer from an assortment of computers, is another notable example.

The upshot of all this is with more, powerful computers there is more and more 
data being created that must be stored. As of November 2004, Lawrence Livermore National Laboratory, where I am a research fellow, has two of the top 5 supercomputers [47]. These are BlueGene/L (\#1) and Thunder (\#5). Simulation runs on Laboratory supercomputers dump terabytes of data. To make effective use of system resources (processing time, memory, disk, cache, network bandwidth), data must be compressed. The data compression process itself should also be implemented to minimize the amount of resources used. This work addresses data transformation and compression/coding with a length constraint. That is, during the transformation or coding process the data values are not allowed to be greater than a certain number of bits in length. This constraint is placed with the intent of limiting the amount of resources required. This is crucial, as in scientific computation any data compression techniques used must be have a minimal impact on the performance of the currently running computation.

\subsection{Motivations}

This work is motivated by two specific, related problems. The first is that disk storage simply cannot keep up with the amount of data dumped during a simulation run. It is not uncommon for simulations to dump terabytes of raw data. I am aware of simulations that have dumped 3, 25, 40, and 98 terabytes. To make more efficient use of storage the data is compressed before being written to disk, but the amount of data is just too much. During the aforementioned 98 terabyte run (on Thunder) the disk was constantly full. This caused other processes to be stopped or delayed while disk space was cleared. Naturally, this is not something we want to occur in a high-performance computing environment.

Data compression can alleviate the problem, but currently the back-end compressor in common use is ZLIB [15]. This gives decent compression rates but is not fast enough. In general, current data compression methods do not meet our current needs. We needed to develop a method that (1) is as fast as possible, (2) has a compression rate comparable to the state--of-the-art coders, and (3) takes up as little resources as possible.

The second problem, related to the matter of there being just too much data, is that when the simulation run is finished and the data stored the scientists typically 
want pictures derived from the data created by the run. These pictures can be obtained by standard computer graphics and scientific visualization techniques, such as isosurface extraction and volume rendering. With terabytes of data on external storage, it is impossible for a scientist to use his or her workstation to perform the rendering, as all the required data is unable to fit on the workstation. Instead, the rendering job is given to a visualization cluster, where each node has a high-end consumer PC graphics card. The job is divided up among the nodes, and the results from each node- found in the nodes' video cards - are composited together into a final image.

The problem then becomes one of how to effectively get the final, rendered image from the remote video card to the user's desktop. The obvious way of doing this is to readback the image into system memory and then use the node's network connection to send the image to the user for display. The problem with this is that if we think of the remote rendering process as a pipeline, it is easy to see that a readback and network transmission stalls the pipeline. Instead of using the node's network interface and performing a costly readback, another method that would leave the video card immediately available is preferable. The most efficient way to do this is to send the image over the card's DVI port [23]. This has several advantages. One is that this is what the DVI port on the card is designed for, and so there is direct access to the frame buffer via the port. The second is that the DVI port has the pixel rate sufficient for displaying high resolution images at an interactive framerate. A 3-channel DVI port can deliver approximately 150 Megapixels per second, while a 6-channel port can deliver about 350 Megapixels per second [23].

We would like to remove the image from the video card via the DVI channel, and have another machine transmit the image to the remote user. To make more efficient use of the network connection we compress the image, and this necessitates transforming the image before compression. Since video GPUs are much more powerful than CPUs it makes sense to perform the transform on the GPU. But the problem is that transforms have dynamic range expansion: if the incoming data is $n$-bit, the transform coefficients are $(n+1)$-bit. A DVI channel is 8-bit, and a typical rendered image is 24-bit (three 8-bit color bands). If each band becomes 9-bit because of the transform, the transmission 
process slows - we have to fit a 9-bit peg into an 8-bit hole.

The key to realizing this whole system is the transform, which must be $n$-bit to $n$-bit.

\subsection{Data Compression}

Data compression is the process of taking an input and finding a unique alternative representation for that input, such that (1) the alternative representation is smaller than the original input and (2) the original data (or a sufficiently close approximation) can be reconstructed from the alternative representation.

Given some input data (image, sound, text) the input may first be transformed. This is done because the transform decorrelates the data, and therefore the transformed data usually compresses better than the original, untransformed data. Once the data is transformed, an estimate is taken of the probabilities of each symbol (number, letter, word) in the data, and from these estimates a code (an alternative representation) is created. Then the data is read piece-by-piece, and for each datum the corresponding alternative representation (a codeword) is output.

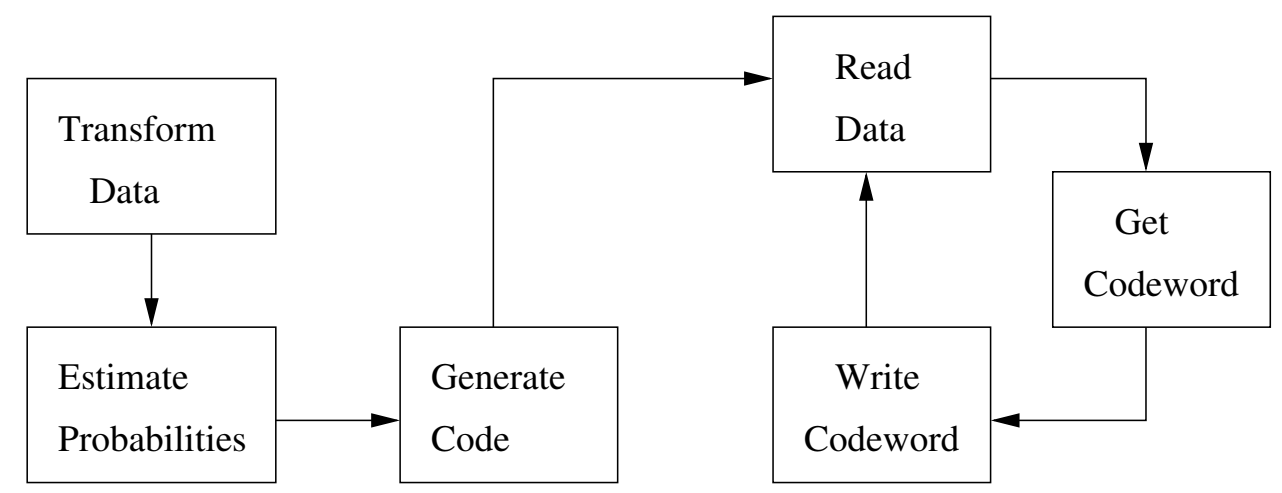

Figure 1.1: Static data compression process.

In the slightly more complex case, a coder may update its probability estimates after encoding each datum, and regenerate the code. This allows the coder to track changing probabilities, and adapt to the varying characteristics of the data. 


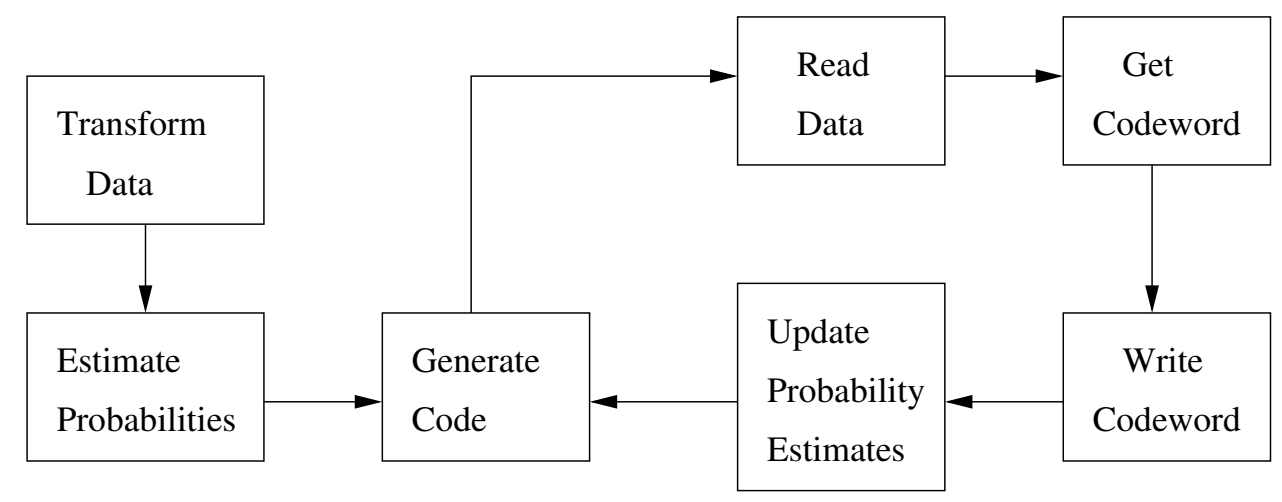

Figure 1.2: Adaptive data compression process.

\subsection{Contributions}

This work develops methods that reduce the number of resources required in the data compression process, with the aim of applying these methods in a high-performance computing environment. The intent is to deliver data compression methods that are competitive with state- of-the-art coders in terms of both execution speed and compression rate.

\subsubsection{Length-limited Variable-to-Variable Length Codes}

The first major contribution of this work is a practical method for generating and applying variable-to-variable length codes in high-performance data compression. Using variableto-variable length codes we are able to create a coder that uses lookup tables for speed, and limit the size of the index into these tables (thereby limiting the size of the lookup table itself) without sacrificing compression efficiency. A coder of this sort is fast and has a compression rate competitive with current state-of-the-art coders.

\subsection{2 $n$-bit to $n$-bit Transforms}

The second major contribution of this work is the development of two $n$-bit to $n$-bit data transforms: the Table-Lookup Haar (TLHaar) and Piecewise-Linear Haar (PLHaar) transforms. PLHaar, in particular, is to our knowledge the only $n$-bit to $n$-bit transform suitable for lossy and lossless coding. 


\section{Part I}

\section{Coding}




\section{Chapter 2}

\section{Information Theory}

\subsection{Messages, Information, and Prediction}

A symbol is a basic unit of representation. Some examples of symbols are bits ( 1 and 0$)$ and letters (e.g. a, b, c, ...). A word or string is any symbol or concatenation of symbols (e.g. 101010, "fred", "zzyzx"). A message or source is comprised of one or more strings ("I am a rock"). A message not explicitly comprised of strings may be parsed into strings as required, e.g. the binary message 10011000110100110 might be parsed into 100110001 10100110.

Every message contains information. Note that in this sense "information" does not indicate the meaning that the message is intended to convey to its recipient. Rather, it indicates the parts of the message that are non-redundant. When we are aware of a redundancy, the redundant parts of a message can be removed without changing the message's meaning. This is because redundancy is predictable. We can identify parts that are predictable, and therefore redundant. These redundant parts can be removed, thus shrinking the size of the message. Later, another person can reconstruct the original message by adding in the redundant bits that were removed. For example, in modern English the letter $\mathrm{u}$ almost always follows a q, when q appears at the beginning of a word. The sentence “Qick Qeens Qietly Qacked!' qoth Qentin” can be reconstructed without difficulty. 


\subsection{Entropy of a Memoryless Source}

Here we examine a memoryless source. A memoryless source can be thought of as a source in which there is a definite probability distribution, but no definite pattern. Such a source might be created by generating a random but weighted bitstream, where there is a set probability of a 1 appearing. Entropy [45] is a measure of the amount of information in a message, or a measure of how much of the message is non-redundant. Information cannot compress, so for data compression purposes a higher entropy value means the message will compress less.

Given that there are $N$ possible symbols in a source, the entropy of the message is computed as:

$$
H=-\sum_{i=1}^{N} p_{i} \log p_{i}
$$

where $p_{i}$ is the probability of symbol $i$ appearing in the message, and the logarithm can be to any base. In data compression applications the log is typically base- 2 , giving $H$ in units of bits per symbol (unless noted otherwise all logarithms here are base-2). Normalized entropy is simply a scaling of $H$ so that it fall in the range $[0,1]$.

$$
\bar{H}=\frac{-\sum_{i=1}^{N} p_{i} \log p_{i}}{-\sum_{i=1}^{N} 1 / N \log (1 / N)}
$$

In its simplest form, encoding a binary source bit by bit, the equation for entropy becomes

$$
H=-(p \log p+q \log q)
$$

where $p$ is the probability of a 0 occurring in the bitstream and $q$ is the probability of a 1. From equations 2.1 and 2.3 we see that the entropy has its highest value when all the probabilities are equal. In this case the source is incompressible. Similarly, when the probability of one symbol is 1.00 (and the others have probability zero) the entropy is zero, indicating that the source has an information content of nothing, and implying that the source can theoretically be compressed to nothing. The entropy curve for the binary case is given in figure 2.1 .

As an example of how entropy is used in data compression, a normalized entropy 


\section{Binary Entropy Curve}

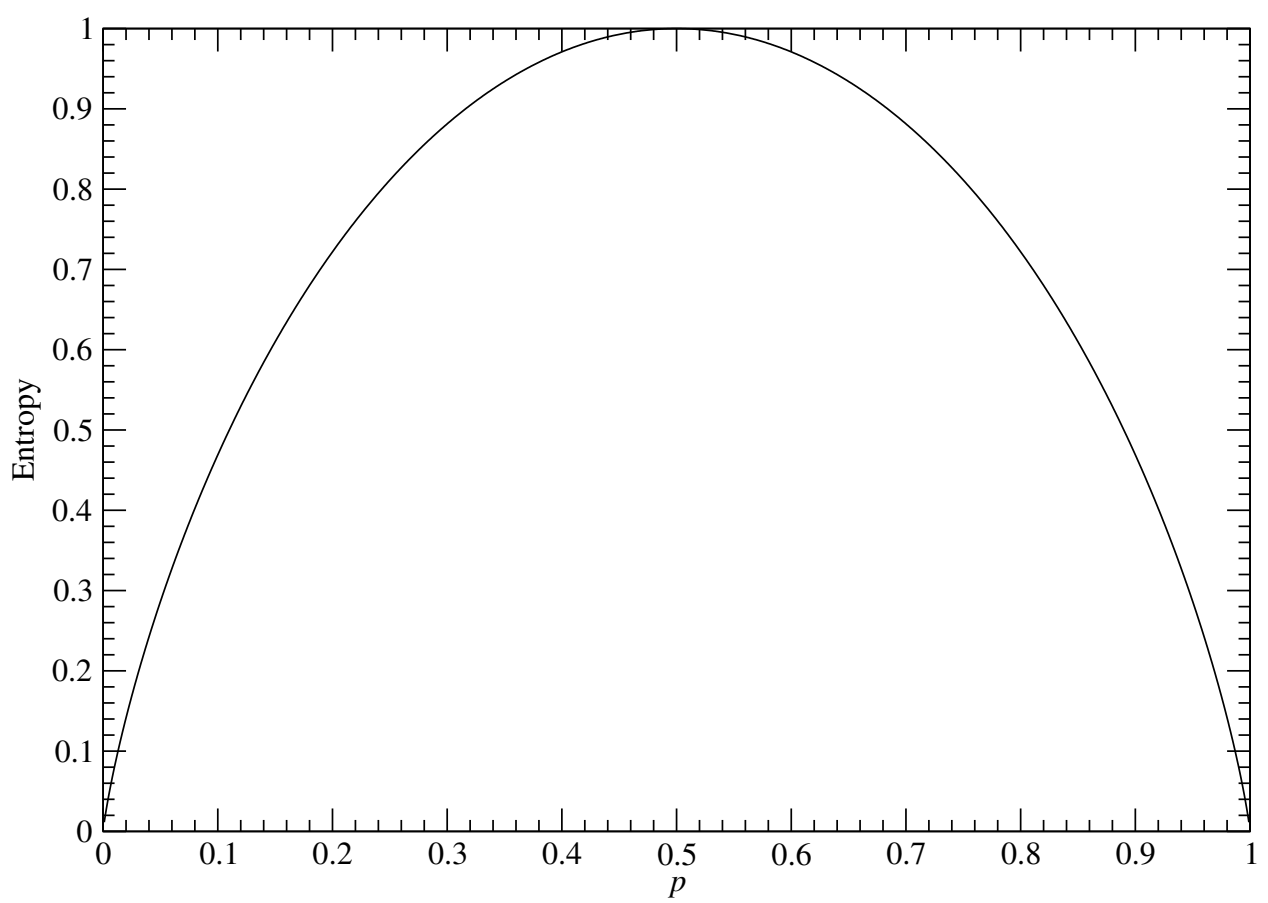

Figure 2.1: Binary entropy curve.

of $\bar{H}=0.42$ indicates that on average for every symbol in the memoryless source, 42 percent of that symbol is used for conveying actual information, and 58 percent is wasted on redundancy. The original message can theoretically be compressed to 42 percent of its original size. Data compression is the process of finding an alternative representation of a given message, such that the alternative representation has a size that is (ideally) as close to the entropy as possible. How close we attempt to come to the entropy is a decision based on compression requirements (why try for a compressed size of $42 \%$ of the original when $66 \%$ is sufficient?) and speed/complexity considerations - often the best compressors are also the slowest.

The entropy equation my also be used to determine how much a source will compress, given a specific method for creating an alternative representation of the source. Equation 2.4 is the basic entropy equation, but with the addition of some accents. 


$$
\tilde{H}=-\sum_{i=1}^{N} \hat{p}_{i} \log \dot{p}_{i}
$$

$\hat{p}$ is the actual probability distribution of the symbols in the source, and $\dot{p}$ is the probability distribution used to create the alternative representation. In this case $\tilde{H}$ answers the question "If the source has an actual probability distribution $\hat{p}$ but we assume it has a distribution of $\dot{p}$, how big will our compressed source be?"

Although an entropy of zero indicates perfect compression, it is of course impossible to compress a source to nothing and still recover it. Practically speaking, there is some overhead incurred in the compression process and as such no source can be compressed to exactly the size indicated by its entropy, although some compression methods come extremely close.

It is also worth emphasizing here that entropy does not measure the absolute limit to which a source may be compressed. Rather, it measures the limit to which a source may be compressed given the method used to identify and remove redundant data. Equation 2.1 indicates that each symbol has a probability $p$. How these probabilities are determined affects the entropy and therefore the size to which the message may be compressed. A common mistake is to compute the entropy using one method, but compress the source using a different method. This can make it seem as if the data compression system is performing much better than it actually is.

As an example, take the binary message 101010101010101010. The entropy of this message, according to the equation for entropy of a memoryless source (2.3), is 1 , because there are an equal number of 1 s and 0 s present. Thus we would say that this binary message is incompressible. But this message is not memoryless! There is a predictable order to the bits in the message. If instead we look at the conditional probability of a bit (e.g. answer the question "what is the probability of the next bit being a 1 given that the current bit is a zero?"), the entropy drops to 0 , which indicates that the message (theoretically!) compresses to nothing. If we use the memoryless source assumption to compute the entropy and then used a data compressor that used conditional probabilities to compress we would incorrectly believe that the data compression system had compressed 
the source to a size far smaller than was possible.

A truly random message is one in which no redundancy can be found, and it is therefore incompressible. This naturally happens when the message is memoryless and all symbols have equal probabilities. Also note that the entropy is accepted as the absolute limit to which a given message may be compressed. There are many people and companies ${ }^{1}$ that claimed to have "broken the entropy barrier" — compressed a message to a size smaller than that indicated by the message's entropy. None of these claims has withstood scientific scrutiny, assuming the claimants allowed such scrutiny in the first place.

\subsection{Conditional Entropy}

Equation 2.3 is sometimes called the zero-order entropy. In this situation to determine the probability distribution of the source the source is scanned and a tally is kept for each symbol encountered. As illustrated previously, given a message we should not just use equation 2.3 to compute the entropy. In many cases we need to use the conditional entropy to more efficiently identify and remove redundancy. In this situation, each symbol has a context in which it appears. Taking our example from above, when we have a message $1010101010101010 \ldots$, we saw that the zero-order entropy is 1, meaning that the source will not compress. To see if we can do better, we create two contexts: context 1 is all bits that follow a 1 bit, and context 0 is all bits that follow a 0 bit. Using one bit of context gives us the first-order entropy. If we group bits according to their context, we get the following two strings:

\section{1: 00000000000 \\ 0: 11111111111}

According to equation 2.3 the entropy of each of these strings is zero, and so each (theoretically) compresses to almost nothing, which means that the original message, using these contexts, can be compressed to almost nothing.

\footnotetext{
${ }^{1} \mathrm{~A}$ listing of some of these claims can be found at http://datacompression.info/IncredibleClaims.shtml
} 
If we have the following string: 110011001100110011001100 the zero-order entropy is again 1. Using the 1 and 0 contexts used previously, we get the following strings:

$$
\begin{aligned}
& \text { 1: } 101010101010 \\
& \text { 0: } 010101010101
\end{aligned}
$$

The zero-order entropy of these each of these strings is 1 , so it looks like in this case the first-order context hasn't solved anything. However, if instead we base the entropy computations on the prior two bits (giving the second-order context), we obtain the following four strings:

11: 000000

10: 000000

00: 111111

01: 111111

And the entropy of each of these strings is again zero. The product of each of these contexts is a memoryless source.

From the data compression system's point of view, a message can be viewed as several different memoryless sources, interleaved together. Compressing using context and conditional entropy is the process of taking a message and decomposing it into several memoryless sources, and compressing each memoryless source separately from the others.

In the third example note that when we used the prior two bits as a context we got four strings, each of entropy zero. In reality, each context has some required overhead. The more context we use the more overhead we incur, and at some point the amount of overhead will be disproportionately large, compared to the compression savings. 


\section{Chapter 3}

\section{Data Compression}

\subsection{Introductory Background}

Recall from chapter 2 that the basic principle behind data compression is to find an alternative representation of a message such that the alternative representation is (1) smaller than the original message, and ideally (2) the alternative representation's size is as close as possible to the size specified by the message's entropy, $H$. The way this is done is to select an alternative representation of each symbol, based on it's probability of appearing. More common symbols receive shorter alternative representations, and less common symbols receive longer representations. How these alternative representations are created is what differentiates the various data compression methods.

As an introductory example, take the two-bit blocks 00, 01, 10, and 11. Any message of $n$ blocks in length created using these four blocks will require $2 n$ bits to represent. Now, suppose we know that for a given (memoryless) message of 256 blocks, the block 00 appears 200 times, the block 01 appears 45 times, the block 10 appears 10 times, and the block 11 appears once. With this information in hand, we compute the probabilities of each block as 00: 0.781, 01: 0.176, 10: 0.039, and 11: 0.004. Using the zero-order entropy equation, we compute the entropy $H=0.934$ bits per symbol. The normalized entropy is $\bar{H}=0.467$, meaning we expect the original message to compress to about $46.7 \%$ of its original size. 
We create a new set of blocks of nonuniform length, and create a lookup table from the old set of blocks to the new one. This mapping from the original blocks to the new ones is a code, and one of the new symbols is called a codeword. If we choose the following mapping:

$$
\begin{aligned}
& 00 \rightarrow 0 \\
& 01 \rightarrow 10 \\
& 10 \rightarrow 110 \\
& 11 \rightarrow 111
\end{aligned}
$$

what happens? The original message took $256 * 2=512$ bits to represent. If we use the above lookup table to replace each original block with a new one, this same message, represented using the new blocks, requires $(200 * 1)+\left(45^{*} 2\right)+(10 * 3)+(1 * 3)=$ 317 bits, which is $61.9 \%$ of the original size.

\subsubsection{Coding Inefficiency}

Since we know the ideal compressed size of the message, and have an actual compressed size, it is helpful to have a way to quantify how efficient or inefficient the code we have is, i.e. when we use the code how close do we come to the entropy?

Given a code and a source message, the coding rate $R$ of a coder $K$ using that code on the message is a measure of how many bits it outputs compared to how many bits were input. $R_{K}$ is computed empirically as

$$
R_{K}=\frac{\text { num_bits_out }}{\text { num_bits_in }} \text {. }
$$

We can compute the theoretical rate as the ratio of the weighted sums of the codewords and the input word lengths:

$$
R_{K}=\frac{\sum_{i=1}^{N}\left|c_{i}\right| p_{i}}{\sum_{i=1}^{N}\left|s_{i}\right| p_{i}}
$$

where $p_{i}$ is the probability of input $s_{i},\left|s_{i}\right|$ is the length of $s_{i}$ in bits, and $\left|c_{i}\right|$ is the length of the codeword assigned to replace $s_{i}$. From this we define the coding inefficiency $I$ of a 
coder $K$ as

$$
I_{K}=\frac{R_{K}-\bar{H}}{\bar{H}} \times 100 .
$$

Inefficiency measures the percentage of bits a coder outputs in excess of that indicated by the entropy. Theoretical inefficiency refers to $I$ computed with a theoretical $R$ (according to equation 3.2), while empirical inefficiency refers to $I$ computed with an empirical $R$ (according to equation 3.1). In our example earlier, the empirical coding rate is $317 / 512=0.619$. Since the normalized entropy was calculated as 0.467 , our code has an inefficiency of $(0.619-0.467) / 0.467 * 100=32.54 \%$.

If a code has a coding rate of $R$ we can compute $H$ at a variety of different input probabilities, and from this determine how efficient we would expect the code to be at compressing bitstreams with various probabilities. Figure 3.1 shows a sample inefficiency curve for a code designed to be most efficient at $q=0.42$. If we wanted to keep coding inefficiency below $1 \%$ we see that we could use this code for $0.37 \leq q \leq 0.46$. Outside of this range we would need a different code, so a coder would need more than one code at its disposal. Figure 3.2 shows the inefficiency curves for three codes. A coder would select one of these three codes to encode a bit based on the current estimation of the probability of a 1 appearing in the bitstream. The minimum of the three curves, shown in figure 3.3, defines the best possible coder inefficiency.

\subsubsection{Lossy Coding}

Lossy coding is the process of encoding a source and throwing away the most redundant or least-significant data. This is usually done when the original data does not need to be preserved intact - only enough data is required for someone to get the meaning of the message, not the complete message. The well-known JPEG specification [28] and image format is an example of lossy coding. Coding where no loss happens is called (predictably enough) lossless coding. The PNG graphics format is an example of this [37]. 


\section{Example Inefficiency Curve}

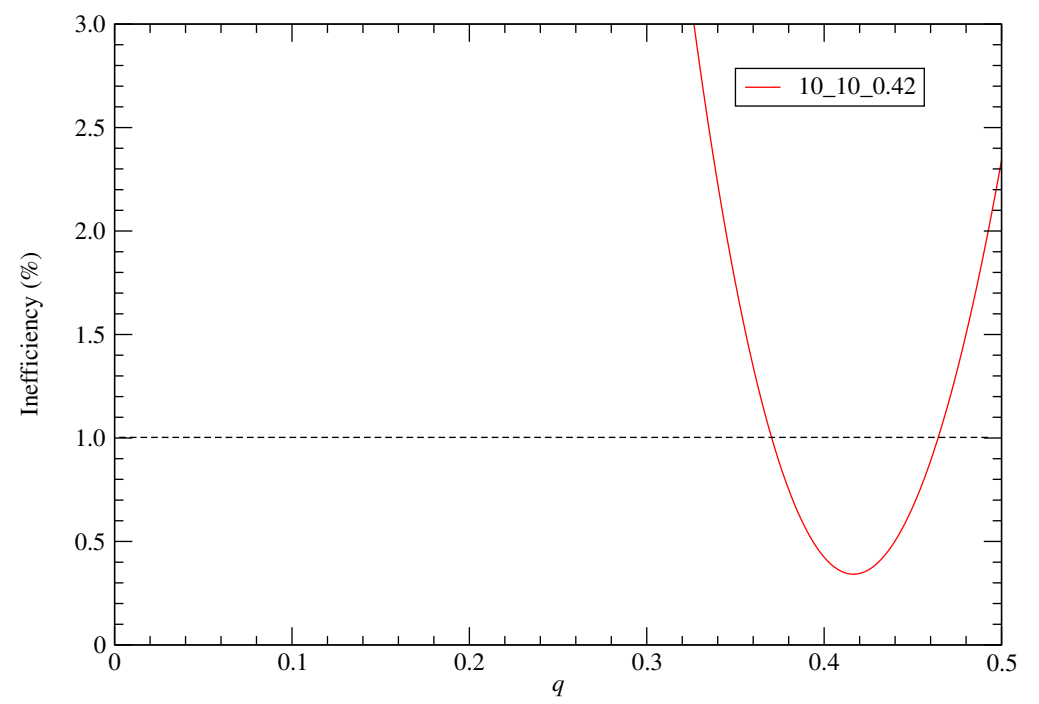

Figure 3.1: Sample inefficiency curve for a code that is most efficient at $q=0.42$.

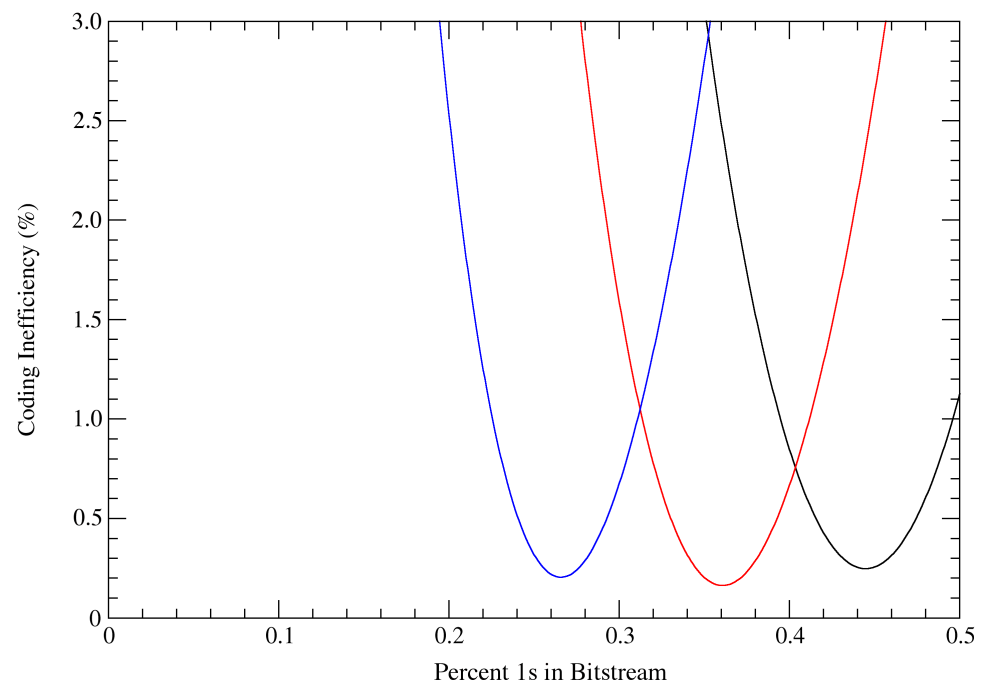

Figure 3.2: Inefficiency curves for three codes. 


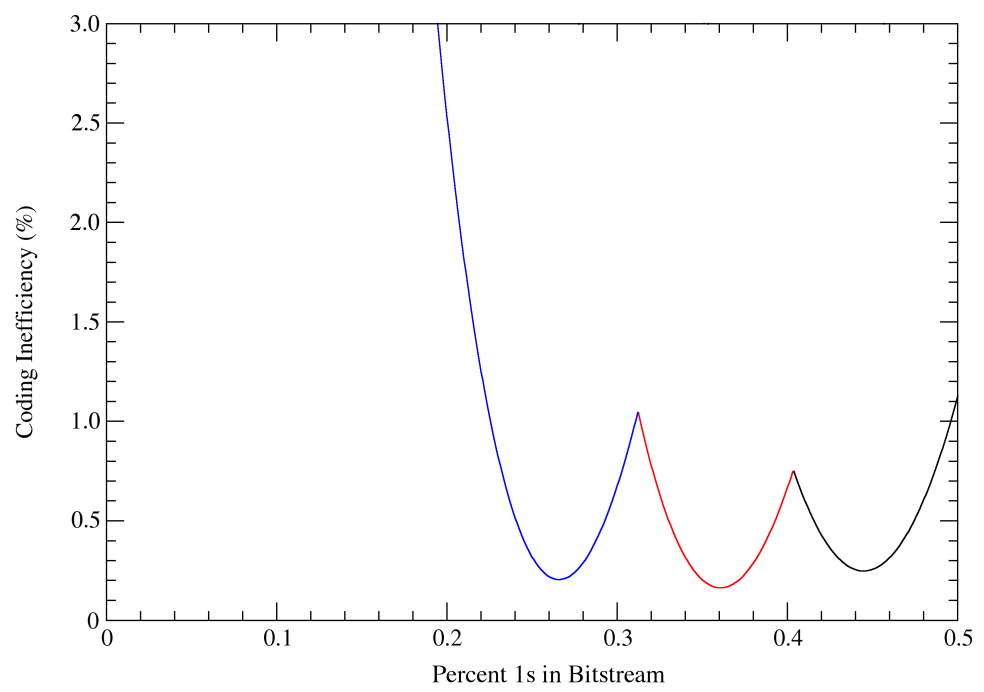

Figure 3.3: Inefficiency curve for a coder using the three codes from figure 3.2.
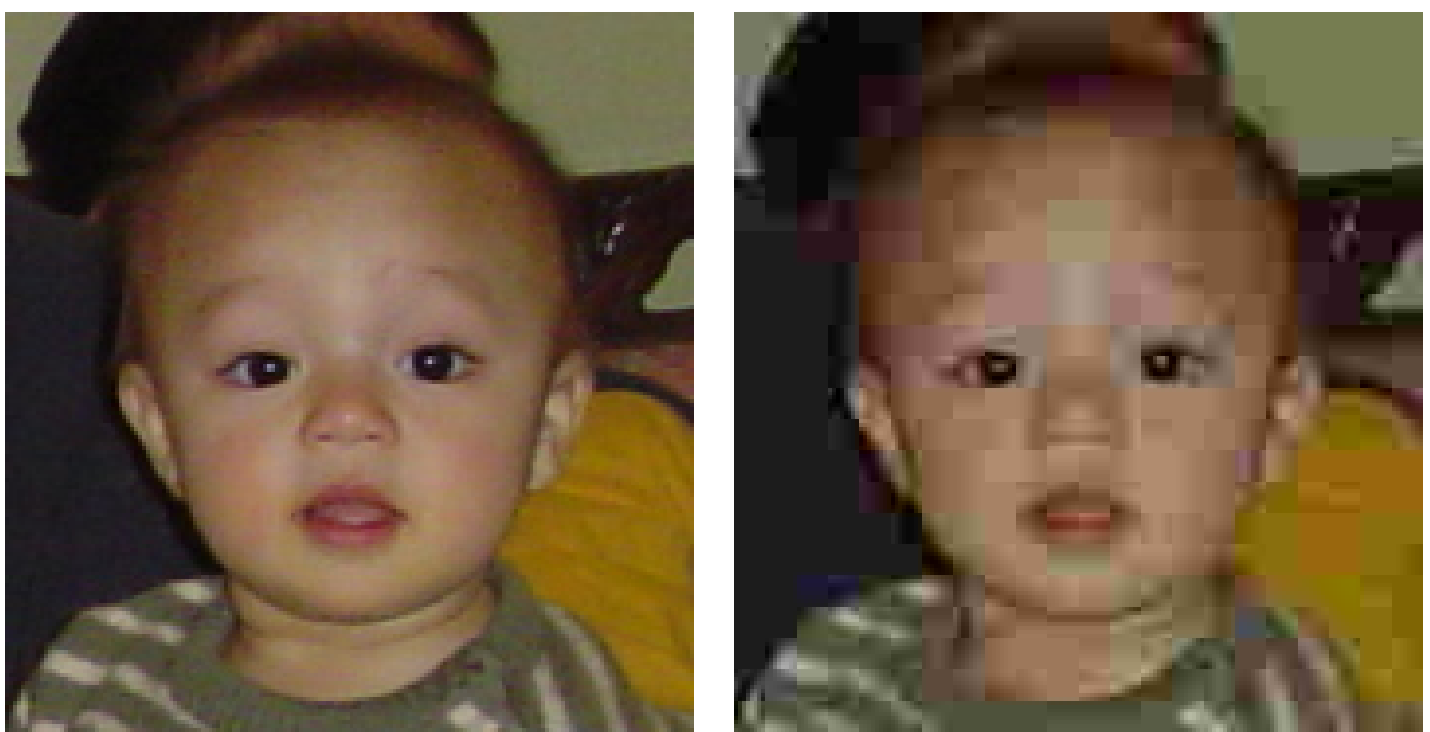

Figure 3.4: An example of lossy coding with JPEG. The image on the left has been coded with little loss, the image on the right with extensive loss. 


\subsection{Some Data Compression Techniques}

The goal of data compression is to find an alternative, shorter representation of a message. How this alternative representation is constructed is what differentiates the different compression techniques. Here we review a few methods that are relevant to this work's contributions. We assume in all cases that we are encoding a binary source, and assume without loss of generality that the more probable bit is a 0 . The probability of a 0 appearing in the bitstream is denoted by $p$, the probability of a 1 by $q$, and $p>q$. We also assume that the binary source is memoryless, that is, it has no underlying structure.

\subsubsection{Run-Length Encoding}

One of the simplest data compression techniques is Run-Length Encoding (RLE). Given a source, a run is any repeated sequence of a single symbol. So for example the binary sequence 100001001 contains one run of 4 zeros, and one run of 2 zeros. An RLE coder scans a source and encodes the runs that it finds by replacing the run with a symbol-count pair, indicating the symbol comprising the run, and how many symbols were in the run.

To simplify our explanation somewhat, since we assume a binary source and $p>q$ we count only runs of zeros. A 1 marks the end of a run.

The standard RLE method starts by first selecting a value $m$, indicating the expected run length. Then a counter $C$ of width $h=\left\lceil\log _{2} m\right\rceil$ bits is created and initialized to zero. As a run is processed, $C$ is incremented. When $C==m$, a 1 is output, $C$ is reset, and counting continues. When the end of the run (a 1 in our case) is detected, a 0 bit is output, followed by the value of the counter. Thus every counter output requires $h+1$ bits.

The fixed-length counter is a weakness in the RLE method. A counter $h$ bits wide can represent a total of $2^{h}$ possible run lengths. If the expected run length $m$ is not

a power of 2 , then there are a total of $2^{h}-m$ unused counter values. This is wasteful, and reduces RLE's efficiency. 


\begin{tabular}{|c|c|c|}
\hline & \multicolumn{2}{|c|}{$\mathrm{m}=14$} \\
\hline Run length & RLE code & Golomb Code \\
\hline 0 & 00000 & 0000 \\
\hline 1 & 00001 & 0001 \\
\hline 2 & 00010 & 00100 \\
\hline 3 & 00011 & 00101 \\
\hline 4 & 00100 & 00110 \\
\hline 5 & 00101 & 00111 \\
\hline 6 & 00110 & 01000 \\
\hline 7 & 00111 & 01001 \\
\hline 8 & 01000 & 01010 \\
\hline 9 & 01001 & 01011 \\
\hline 10 & 01010 & 01100 \\
\hline 11 & 01011 & 01101 \\
\hline 12 & 01100 & 01110 \\
\hline 13 & 01101 & 01111 \\
\hline Total & 70 bits & 68 bits \\
\hline
\end{tabular}

Table 3.1: A comparison of RLE and Golomb codes for $m=14$. The Golomb code uses less bits.

\subsubsection{Golomb Coding}

An improvement to the standard RLE method is Golomb coding [19]. Golomb coding seeks to improve the efficiency of the standard RLE method by using a variable-length counter. Given $p$, the expected run length is computed as $m=-\log 2 / \log p$. Golomb's observation was that if we select the smallest positive integer $k$ such that $2^{k} \geq 2 m$, then the first $2^{k-1}-m$ counter values can be represented in $k-1$ bits, while the remaining must be represented in $k$ bits. This is an improvement over standard RLE, which must represent all counter values in $k=h+1$ bits. One of the examples in [19] is for $m=14$. Table 3.1 reproduces part of that example, comparing the Golomb code with a standard RLE code. We see that in this case the Golomb code uses 2 bits less than the standard RLE code. This may not seem like much, but for a bitstream with millions of bits the savings will be significant. In general, given $m$, the Golomb savings over standard RLE is $2^{k-1}-m$ bits.

Figure 3.5 gives the inefficiency curve for Golomb codes. From this we see that the codes (as could be expected) are most efficient when there are long runs (at lower 
Golomb Coding Inefficiency

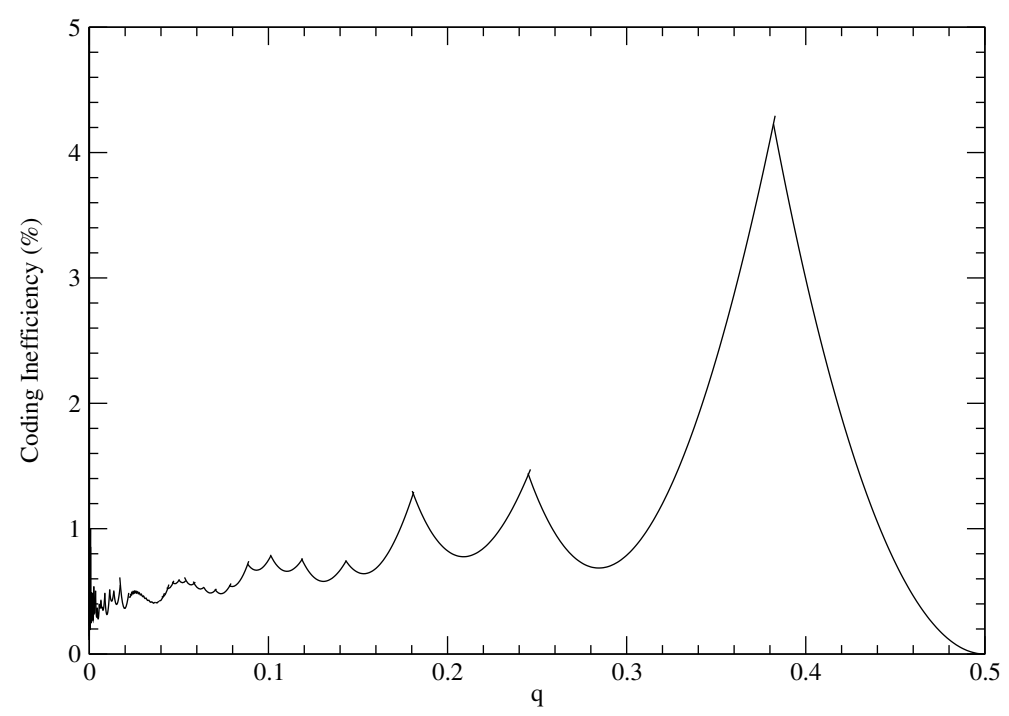

Figure 3.5: Inefficiency curve for Golomb codes. Note the great inefficiency at about $q=0.38$.

values of $q$ ). The codes are not as efficient for $q \geq 0.18$. In particular, there is a large inefficiency at about $q=0.38$.

The problem with any RLE method is that it is not efficient unless lots of long runs are expected in the source. Even with a variable-length counter there is overhead incurred, and that overhead is significant if the runs are not sufficiently long.

\subsubsection{Huffman Codes}

The Huffman algorithm [27] is not a coding method as such, but a way of generating a code given a set of input symbols and a probability distribution. After the code is generated each symbol in the input is replaced with the appropriate codeword, resulting in compression.

The process is illustrated in figure 3.6. Given a set of symbols and a probability distribution, the two least probable symbols are chosen and made leaves of a tree, with the least probable symbol on the left. The tree is returned to the set. Then, of the symbols and trees in the set, the two least probable are chosen and formed into a tree, with the least probable on the left. The tree is returned to the set. The process repeats until only 


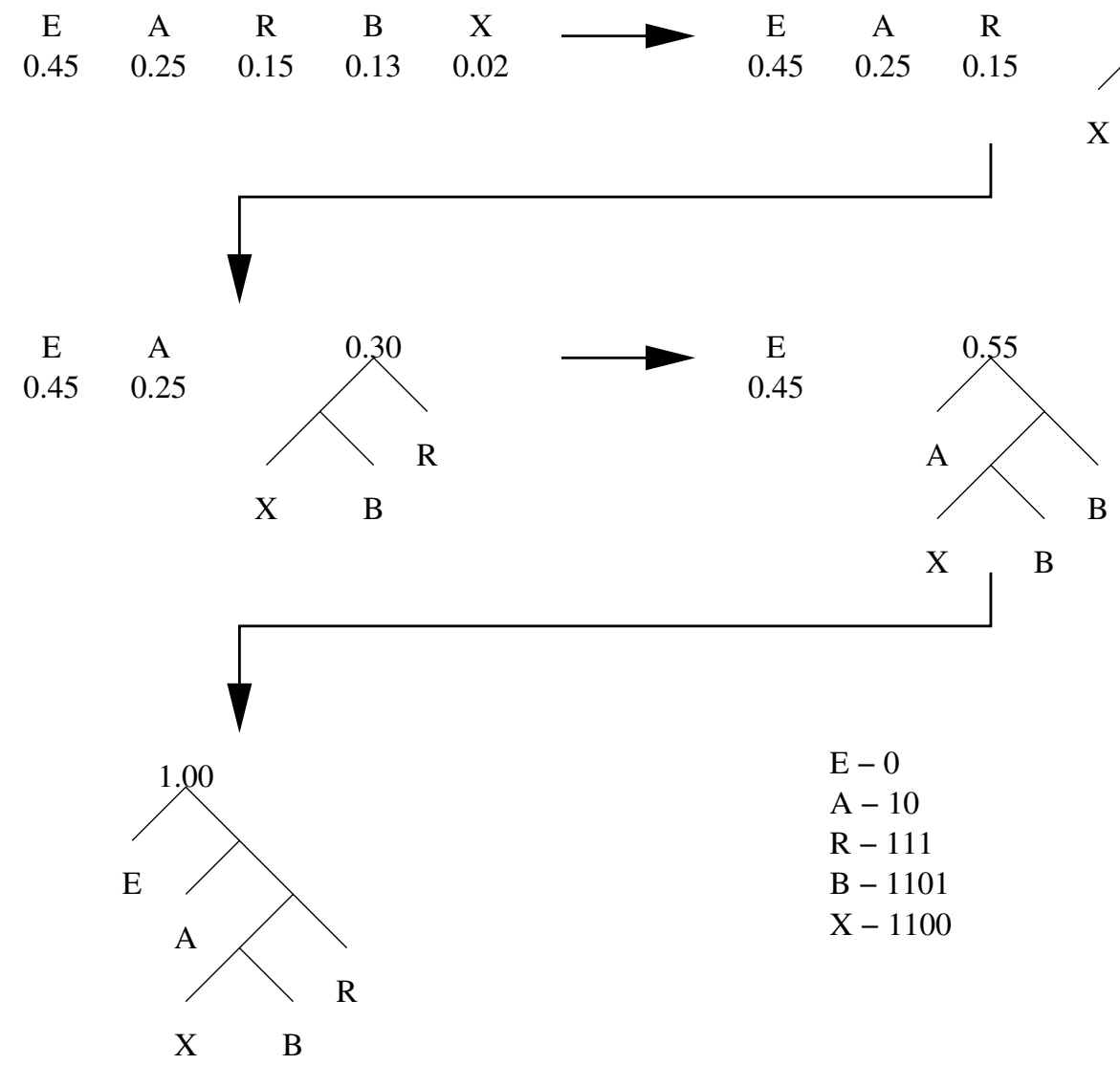

Figure 3.6: An illustration of the Huffman code generation process. A left branch is a 0, a right branch is 1 .

one tree remains. The tree is traversed, the path from the root to each leaf giving the code for that symbol.

There are several important characteristics of the Huffman algorithm. The first is that the Huffman algorithm cannot produce a code that has fractional bit widths (the Arithmetic codes, to be discussed below, can). This means that when using a Huffman code it is not possible to have a compression rate smaller than 1 bit per symbol. If the inputs are all $W$ bits wide the maximum possible compression rate is $1 / W$. The second is that in a Huffman code no codeword is a proper prefix of any other codeword. This allows for unambiguous decoding. The third is that for codes of this type (code tree, no fractional bit widths) the Huffman codes are optimal. The fourth is that if there are $N$ inputs the longest possible code length is $N-1$, although this is unlikely to occur. 


\subsubsection{Parse Tree Methods}

Parse tree methods are methods that take a source and parse it into symbols and words. There are different types of parse-tree methods, but their underlying mechanics are the same: a source is parsed into words, and these words are then encoded. One of the major benefits of parse tree methods is that an extremely fast coder using them is easily implemented using table lookups.

Note that from a certain standpoint the run-length codes may be considered parse-tree methods. We separate run-length codes from parse tree methods because the mechanics used to create the codes are significantly different from each other.

\section{Block-to-variable Length Codes}

The Block-to-variable length (BV) codes parse a source in fixed, uniform-length strings or blocks. Given $p$ and $q$, a probability for each block is computed and codes are generated, assigning the shortest code words to the most probable blocks and the longest code words to the least probable blocks. How these codes are created is a matter of personal preference and requirements, but the typical methods are the Huffman algorithm [27] and canonical codes [39].

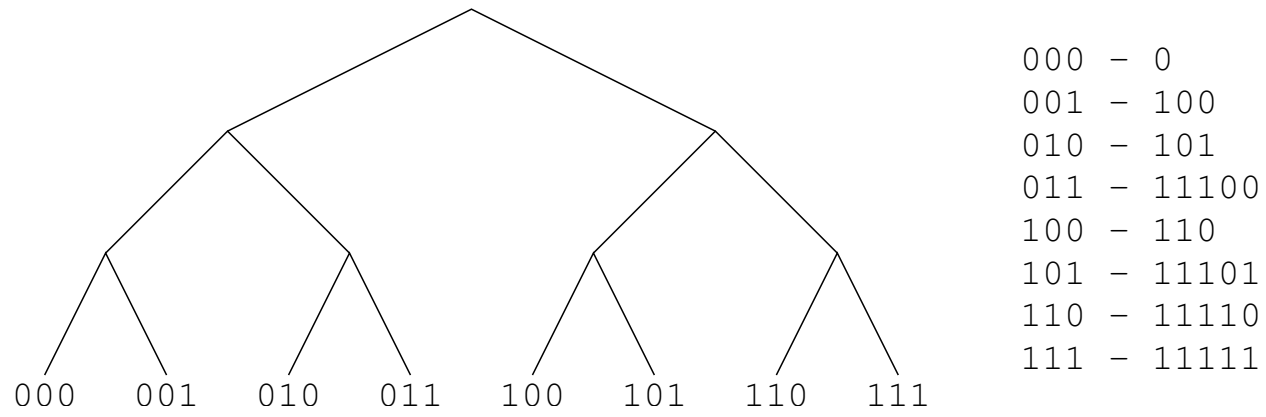

Figure 3.7: A BV code parse tree and code table. $B=3$, and codes are generated using the Huffman algorithm. 


\section{Variable-to-block Codes}

Variable-to-block (VB) codes, as their name implies, parse a source in words whose length may vary one from another and output codes that are all of uniform length. The standard example of a method for generating a VB code is the Tunstall algrithm (see $[10,11,17]$ ).

The main advantage to the Tunstall codes is that since the output code length is uniform there is no special algorithm required to assign the codewords. All $2^{B}$ codes are unique and therefore uniquely decodable, so any method desired can be used. One possibility is to assign sequential codes to the symbols in nonincreasing probability order. This ensures that when decoding the tables or parts of the decoding tree most likely to be accessed are grouped together and kept in cache memory. The main disadvantage to the Tunstall method is that the coding rate may not be the best obtainable. If the longest input string from the parse tree is $W$ bits, the best possible compression rate is $B / W$. It may be that if the code lengths were allowed to vary, thus giving the most probable string a shorter codeword, a better compression rate would be possible.

\section{Variable-to-variable Length Codes}

Variable-to-variable length (VV) codes parse a source in variable-length words and output codes that may vary one from another in length. The typical way of describing VV codes is a Tunstall-Huffman pairing: the Tunstall algorithm is used to create the parse tree, and the Huffman algorithm is used to create the codes. This may be overly restrictive, as it limits the number of leaves on the parse tree to powers of 2. A slightly modified method can be used where the extension process terminates when the desired number of leaves (or some other criteria) is reached.

The main advantage to VV codes is that they may obtain a better compression rate than other parse tree coding methods. Their disadvantage is that the only way to find an optimal VV code is through an exhaustive search. VV codes will be discussed in greater depth later. 

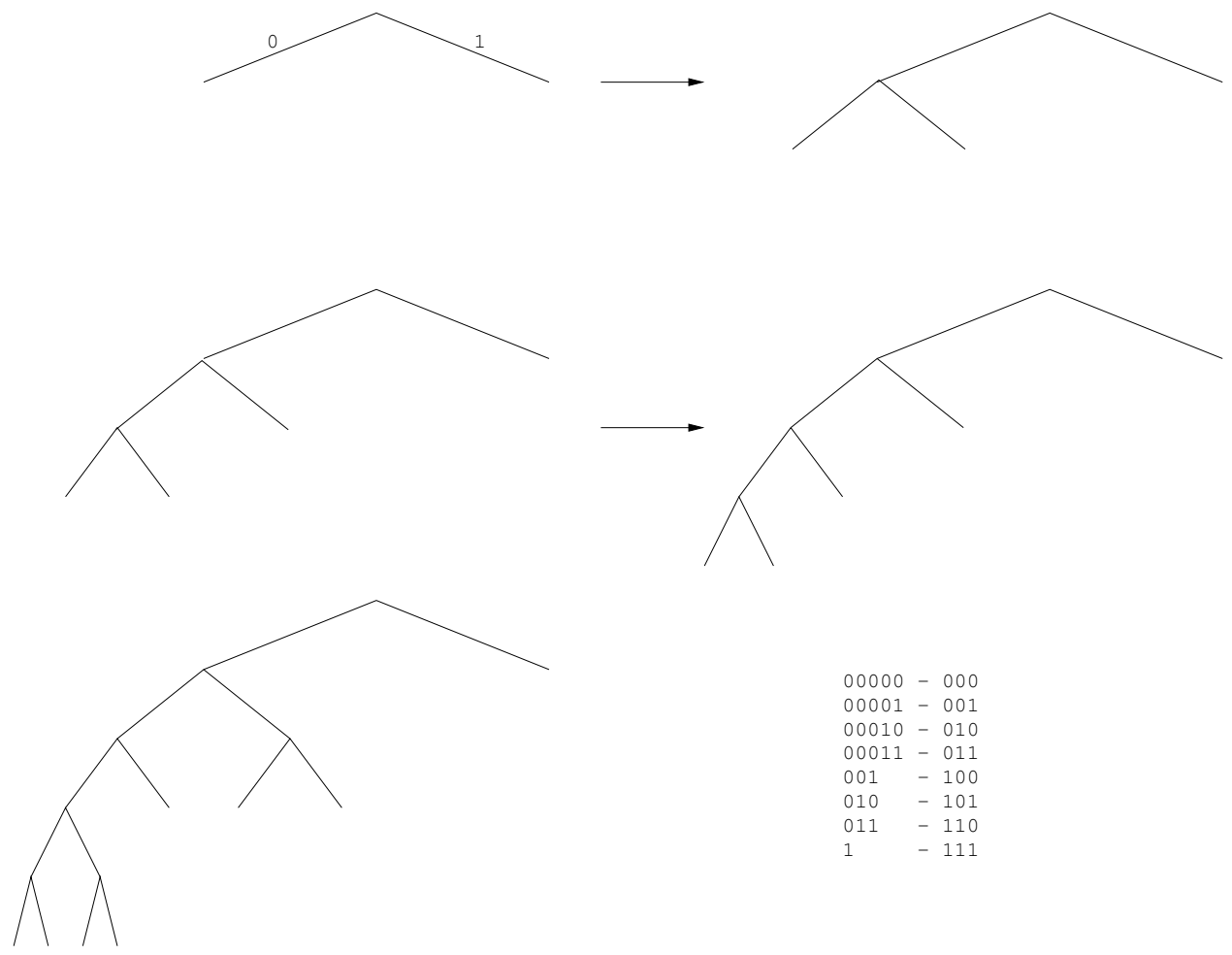

$$
\begin{aligned}
& 00000-000 \\
& 00001-001 \\
& 00010-010 \\
& 00011-011 \\
& 001-100 \\
& 010-101 \\
& 011-110 \\
& 111
\end{aligned}
$$

Figure 3.8: The creation of a VB parse tree, by extending and splitting the most probable leaf.

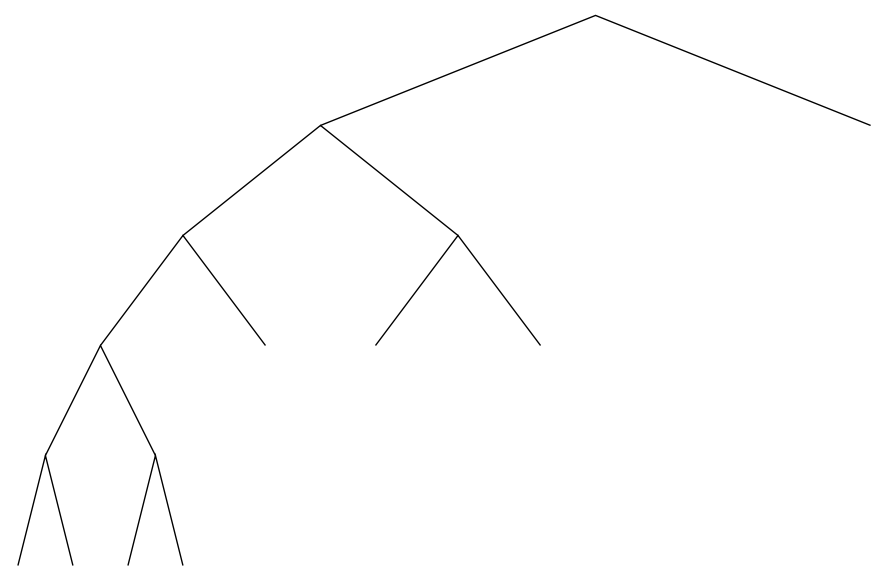

$$
\begin{array}{ll}
00000 & -0 \\
00001-10 & \\
00010 & -1100 \\
00011-11110 \\
001-11100 \\
010-11101 \\
011-1101 \\
1 & -1111
\end{array}
$$

Figure 3.9: A VV parse tree (created using the Tunstall algorithm) and its code table. Codes are generated using the Huffman algorithm. 


\subsubsection{Arithmetic Codes}

Arithmetic Codes [61] are the codes that come closest to the theoretical entropy of a source. As its name implies, an arithmetic code represents a source as a real-valued number computed out to many bits of precision. As each symbol of the source is encoded, more precision is added to the number. The number created for each source is unique, and so from each number it is possible to decode the source.

The binary arithmetic coding process is conceptually simple. The arithmetic coder begins with a full probability range, and divides it into two subranges sized according to $p$ and $q$. A subrange is selected based on the actual bit encoded, then that subrange is divided into two subranges, and the process repeats. The main advantage to the arithmetic codes is they are able to represent fractional bit widths. Their main disadvantage is their speed: they are very slow.

There are approximation methods for arithmetic coding. These methods, while not pure arithmetic codes and therefore not as efficient, have a very good coding rate and a much improved execution speed. Some examples of these types of coders are the $\mathrm{Q}-$ coder [36] and its variants, and the ELS [57, 58] and Augmented ELS [59] coders. Ono et al [35] describe MELCODE, which is a method of using a run-length type code to approximate an arithmetic code. Bottou et al [9] describe the Z-Coder, which is another run-length approximation of an arithmetic code. Due to patent concerns the original implementation

of the Z-Coder has been modified, and this version, called the $\mathrm{Zp}$-Coder, is currently used.

Most arithmetic code approximation techniques couple a probability estimation state table to the coder. The current state gives the current probability estimation (this is usually in some form that the coder can use to compute the code quickly) and the state transitions based on whether the current bit is a 1 or a 0 . 


\section{Chapter 4}

\section{A High-performance Encoder Using Variable-to-variable Length Codes}

\subsection{Introduction}

Our first contribution is a prototype coder for high-performance computing [40]. This was created to address one of the motivations referred to in Section 1.2: when a supercomputer is running a simulation massive amounts of data are generated, and the current methods for handling the data (including compression) are not fast enough. The data cannot be dumped from the machines fast enough, and the resulting data can overload storage if not compressed. Current compression libraries are too slow. The question I needed to address was, can we create a coder that is significantly faster than current coders, but also gives a competitive compression rate?

The first issue was which basic coding method to use. The current coder in use is the ZLIB library [15], which is rather slow. Typically, there is a speed-compression tradeoff. Slower methods tend to achieve better compression rates (a good compression rate requires techniques that are more computation-intensive), and faster methods worse rates. Pure arithmetic coders obtain the best compression rates, but they are too slow. 
Run-Length codes, especially the Golomb code, are fast and efficient only when the bit probabilities are such that a significant run length is expected.

In terms of speed, a method using direct lookup tables that contain the codewords would be hard to beat, as there is significantly less computational overhead. I decided to explore table-lookup methods, and the process of designing a coder became one of getting the best compression performance using tables.

\subsection{Encoding Using Table-lookups}

\subsubsection{A Simple, Fast Process}

The codes best suited for table-lookup methods are the parse tree-based codes (BV, VB, VV). Given a code, the coding process is simple, as illustrated for encoding in figure 4.1. Given an input and a window size of $W$ bits, we (1) examine the next $W$ bits on the input, using them as an index into a lookup table. The table entry gives values for $l$-the number of bits actually used from the input, $c$-the length of the codeword, and the codeword itself. We then (2) shift $l$ bits off of the input, (3) shift the output by $c$ bits, and (4) place the codeword on the output. The process is simple, and the coding loop is very tight. As long as the lookup table is not excessively large and does not take up too much cache space the coding process will be fast. The table size is a function of the size of the index $W$-there must be $2^{W}$ entries in the lookup table. For a fast, direct-lookup scheme $W$ must be as long as the longest string on the parse tree (when encoding) and as long as the longest codeword when decoding.

\subsubsection{Limiting Table Size}

If a codeword (read input string here also - the reasoning is the same in either case) is excessively long the lookup table will have too many entries and be excessively large. For example, assume that each table entry is 32 bits wide. A longest code length of 16 bits yields a decoding table that is 256 kilobytes in size. This table will easily fit in cache memory. If the longest code length is instead 20 bits long, the table grows to 4 megabytes 


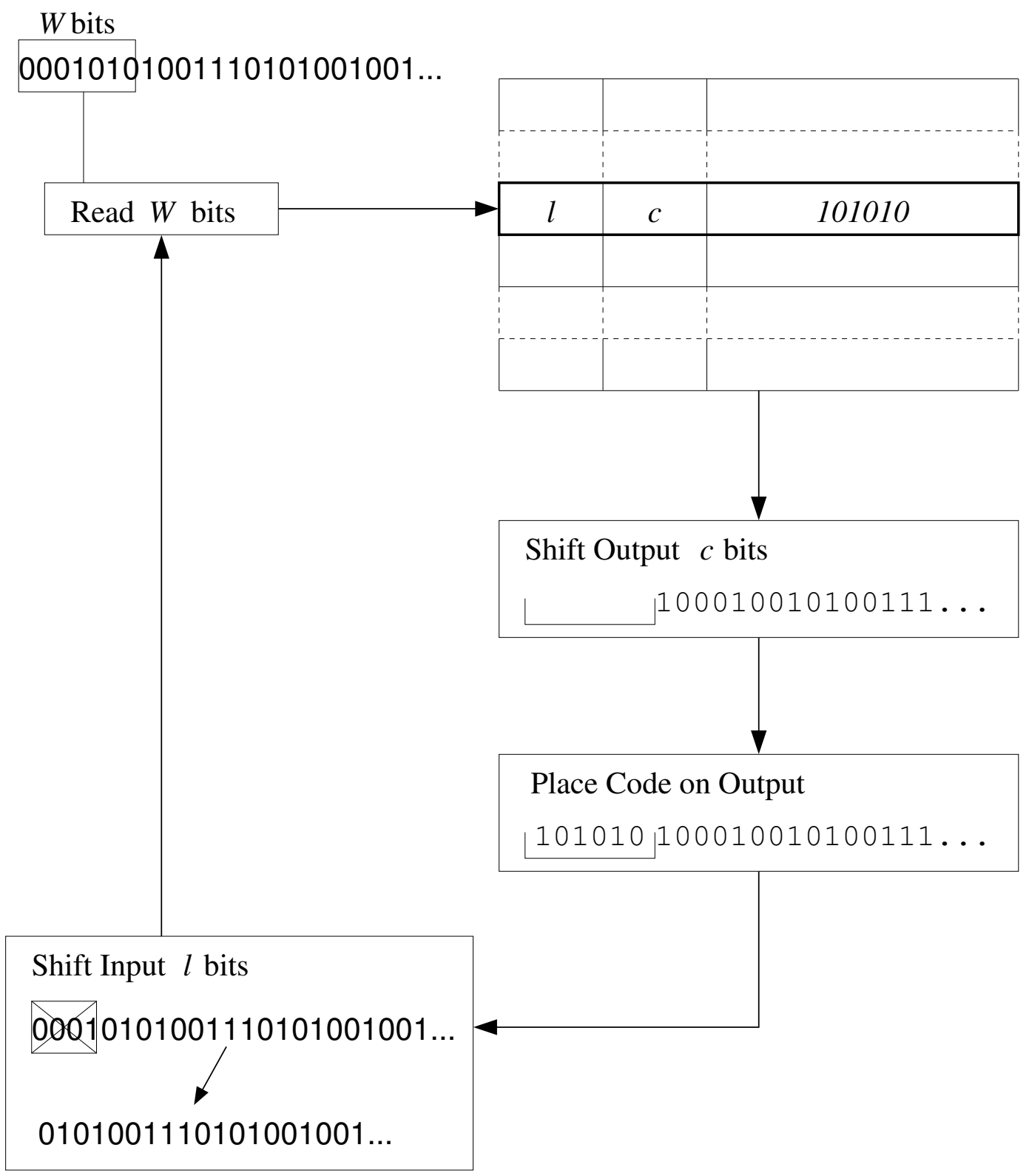

Figure 4.1: Encoding via table-lookup. 
in size. Both of these code lengths are possible with a reasonable input set size, depending on the probabilities of the symbols, but a difference of only 4 bits in code length results in a $1600 \%$ increase in table size.

Remember that in the worse case if there are $N$ possible inputs the longest possible code length is $N-1$ bits. While this longest code length is obtained only in very extreme cases, it is a reminder that table size can be a nasty issue if care is not taken to keep the code lengths short.

\section{Block Shortening}

The simplest technique for shortening code (or input string) lengths is to iteratively shorten the block size and regenerate the codewords (when using BV codes) or the parse tree (when using VB codes), terminating when the longest code or string is less than or equal to some threshold $C$. This technique is simple, but as the block length is shortened the overhead of the code increases rapidly (recall for example that a BV code can never have an overall compression rate better than the best input/output pair).

\section{Package-Merge Algorithm}

A more intelligent approach is to use the Package-Merge Algorithm [32] or similar algorithms. We do not review these methods here, but they basically use a greedy approach where the longest code length is shortened and the code tree is readjusted to accommodate the change. This process repeats until all code lengths are within some specified limit. This process naturally reduces the overall compression efficiency of the code, but since the longest code lengths (corresponding to the least-likely inputs) are modified first the decrease in compression efficiency is kept as small as possible. In practice the PM algorithm does extremely well, and if the algorithm isn't given a difficult set of requirements (such as an input code with very skewed probabilities and a very small longest code length) it is often able to create a length-limited code with only a small decrease in compression rate. Figure 4.2 shows the coding inefficiency for block-shortened Tunstall, block-shortened BV Huffman, and BV Huffman codes with code lengths limited via the 
Package-Merge algorithm. Inputs were limited to 15 bits maximum, outputs were limited to 13 bits, and the most optimal codes were chosen from within this search space. As expected, length-limiting via the PM algorithm is the best method of the three.

\section{Use a VV Code}

The third alternative is to use a VV code. Code lengths are dictated by the number of input strings and the probabilities associated with them. Being able to create a parse tree with variable-length strings gives us the ability to potentially create a parse tree that will result in a code with the desired code lengths, while providing a good compression rate. Figure 4.3 shows the most optimal variable-to-variable length codes created using the merging process (to be discussed in section 4.3.2), with the same limits as the codes in figure 4.2. The improvement is dramatic.

\subsection{Generating a Length-limited VV Code}

\subsubsection{Extension}

To date it seems that most (to my knowledge, all) of the published work in creating VV codes involves the extension process, one method of which was described in Section 3.2.4. To review, the most basic way of creating a VV code uses the Tunstall extension approach to create the parse tree, extending the leaf corresponding to the most probable string, and uses the Huffman algorithm to generate the code words. This is overly restrictive, as it requires that the number of leaves on the parse tree be a power of 2 . A variant of this method allows the number of leaves to not be a power of 2 , the extension process terminating based on some other criteria. Intuitively, the Tunstall-based extension approach is a good one: by creating longer strings of more-likely bits (and giving them shorter codewords) and shorter strings of less-likely bits (and giving them longer codewords), the coding rate is more likely to be closer to optimal. However, a Tunstall-like extension approach does not guarantee optimality, nor does it guarantee the best possible coding rate compared to extending leaves in a different manner. 
Inefficiency of Length-Limited Codes

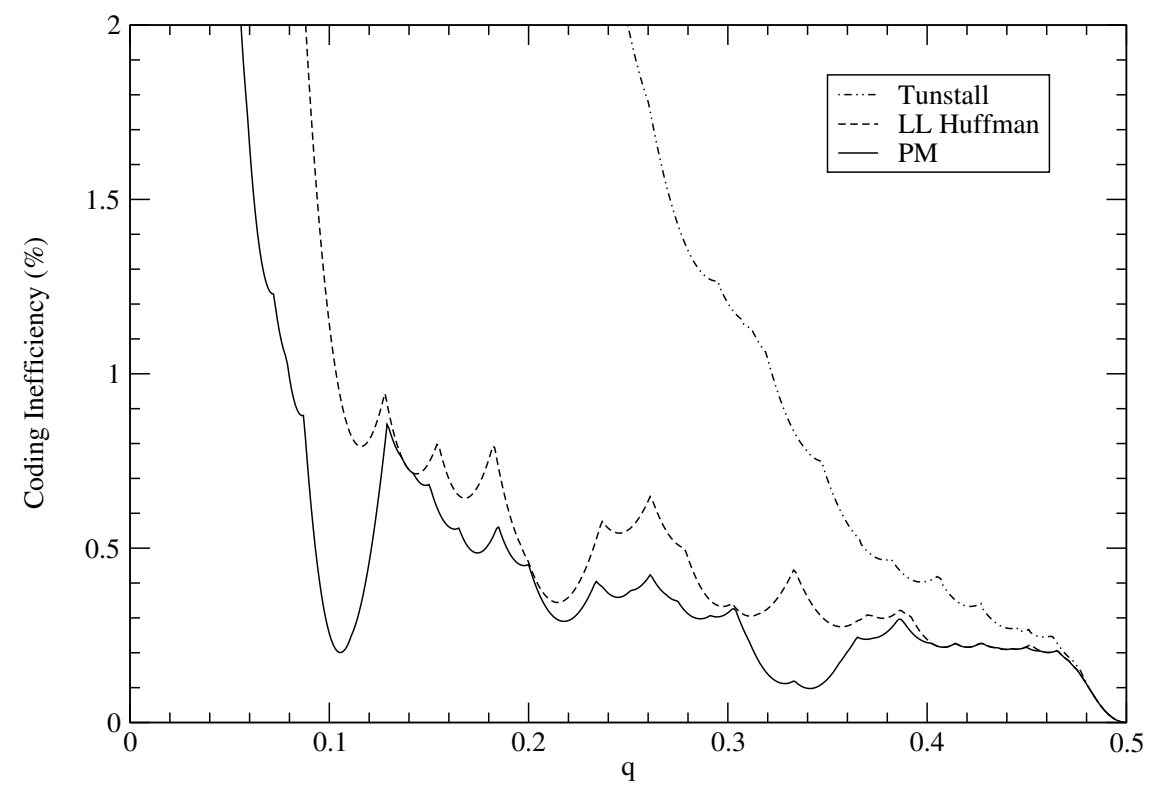

Figure 4.2: Coding inefficiency of length-limited codes.

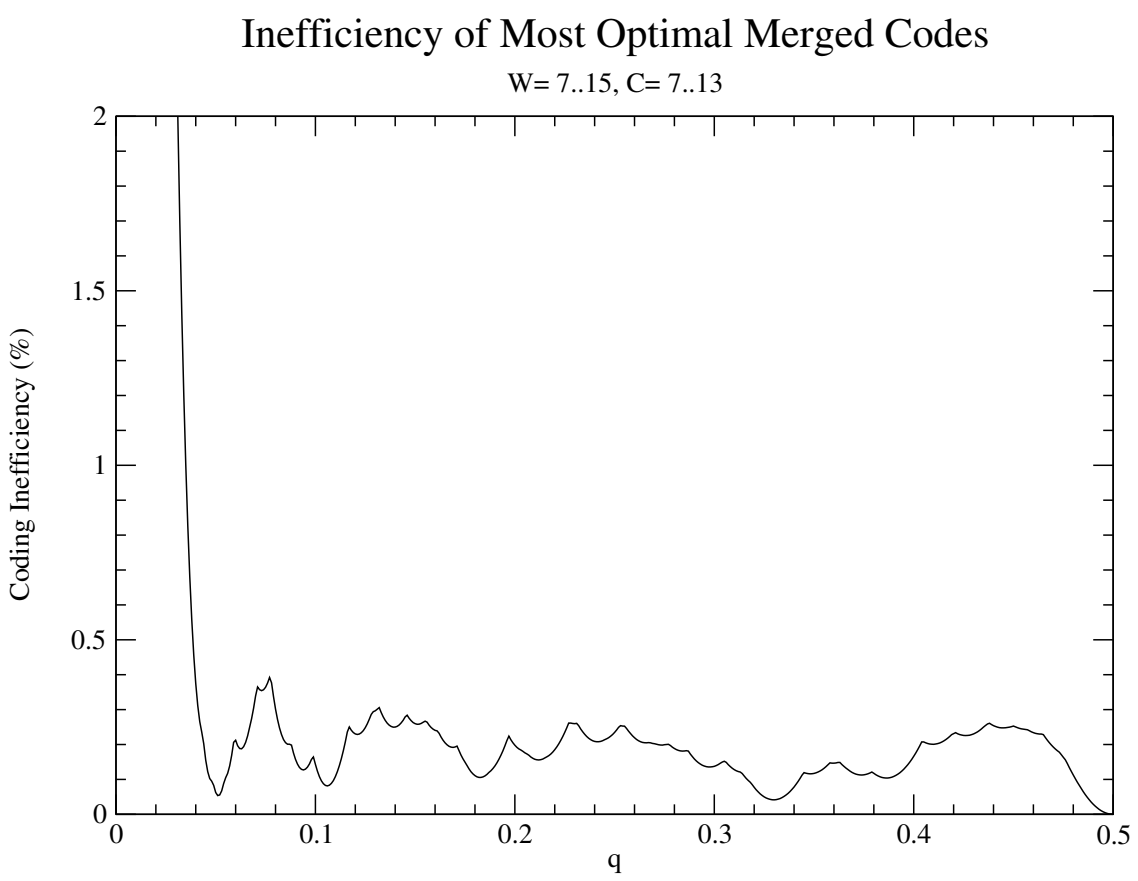

Figure 4.3: Coding inefficiency of some VV codes. 
Much work in VV code research has gone into examining the current parse tree and then deciding which leaf on the parse tree is to be extended (see [16, 18, 48, 49] for example). The research met with limited success, and indicates that even when starting from an optimal parse tree, it is not always possible to obtain another optimal parse tree by extension.

In short, creating optimal VV codes is a notoriously difficult process, and all research indicates that an exhaustive search remains the only method to find an optimal VV code [16, 48, 49]. Peter Stubley, whose doctoral dissertation examined VV codes, believes that the problem of finding an optimal VV code is NP-Complete [50], although he has no formal proof.

The bright side to all of this is that while it is difficult to find an optimal code, finding a code that works well enough is a much simpler process.

\subsubsection{Source String Merging}

Our original work in VV codes derived solely from our need to create length-limited codes. We had BV codes which we intended to use in our coder, but which were not suitable due to the excessively long code lengths, and we needed a way to shorten the codes. We observed that by reducing the number of things to be encoded (in this case, leaves on the parse tree) we could potentially reduce the lengths of the codewords. As the coding rate is dominated by the more-likely leaves of the parse tree, it made sense to eliminate, or modify, the least-likely leaves, as this would have the least detrimental effect on the coding rate.

We chose to create VV codes through a greedy process of contraction, or merging. In this approach, a block length $B$ is selected, and a full parse tree with $2^{B}$ leaves is created, where all strings are of length $B$. Code lengths are generated for each string, using the Huffman or another equivalent algorithm. We then follow a greedy approach: while the longest code length $L$ is greater than some limit $C$, the least-likely leaf on the parse tree is merged with its sibling into their parent node, which becomes a leaf. Code lengths are re-generated, and the process repeats until $L \leq C$. In essence the merging process takes 
a BV code and transforms it into a VV code. The process is illustrated in figure 4.4, and an actual parse tree generated by this method is in figure 4.5 .

\subsection{Properties of the Greedy Merging Process}

The first thing to note about this process is that the greedy algorithm will always choose a leaf with a sibling. The proof is simple. Assume that we have subtree taken from a parse tree, structured as in figure 4.6. If we let $s$ denote the path to the root of this subtree, and $w$ the weight of that path, we see that the leaves of the parse tree have strings $s 00, s 01$, and $s 1$. Weights are respectively $w p p, w p q$, and $w q$. Since $w q>w p q$ and $w p p>w p q$, leaf $s 1$ can never be selected before $s 01$. Any other tree configuration yields a similar proof.

Another property that is simple to prove is that the merging process will always choose a leaf corresponding to a string ending in a 1. For any given leaf pair, the strings corresponding to the leaves are identical, except in the last bit position. If the weight of the path to the pair's parent node is $w$, the weight of the path to the string ending in a 0 is $w p$, and the weight of the string ending in a 1 is $w q$. Since we assume WLOG that $p>q, w p>w q$, and the selected leaf - the leaf of least weight-must be the 1-ending leaf.

Recall that given a parse tree that recognizes $N$ strings, the coding rate for the resulting code a code is

$$
R=\frac{\sum_{i=1}^{N}\left|c_{i}\right| w_{i}}{\sum_{i=1}^{N}\left|s_{i}\right| w_{i}}
$$

where $\left|s_{i}\right|$ is the length of string $i, w_{i}$ is the string's weight, and $\left|c_{i}\right|$ is the length of the codeword assigned to string $i$.

We demonstrated that at each merging step the string selected for merging will end in a 1 . We refer to this string as $s_{1}$, its 0 -ending sibling as $s_{0}$, and the merged string as $s$. Their respective weights are $w q, w p$, and $w$. At each merging step, given $s_{1}$, the denominator's value changes in a predictable fashion. If $x$ is the value of the denominator - the weighted sum of the string lengths - before a merging step, and $x^{\prime}$ the 

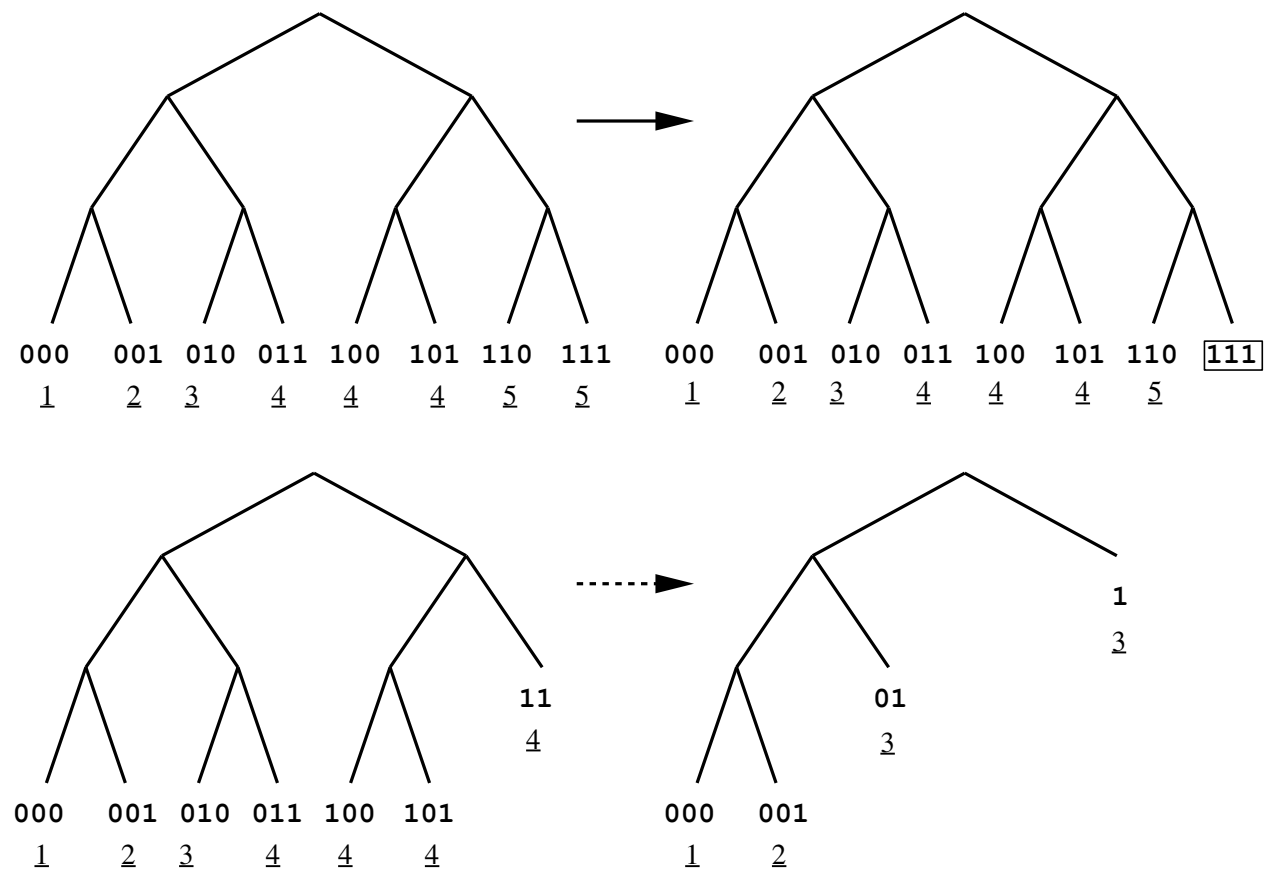

Figure 4.4: Source String Merging. The underlined numbers indicate the code lengths.

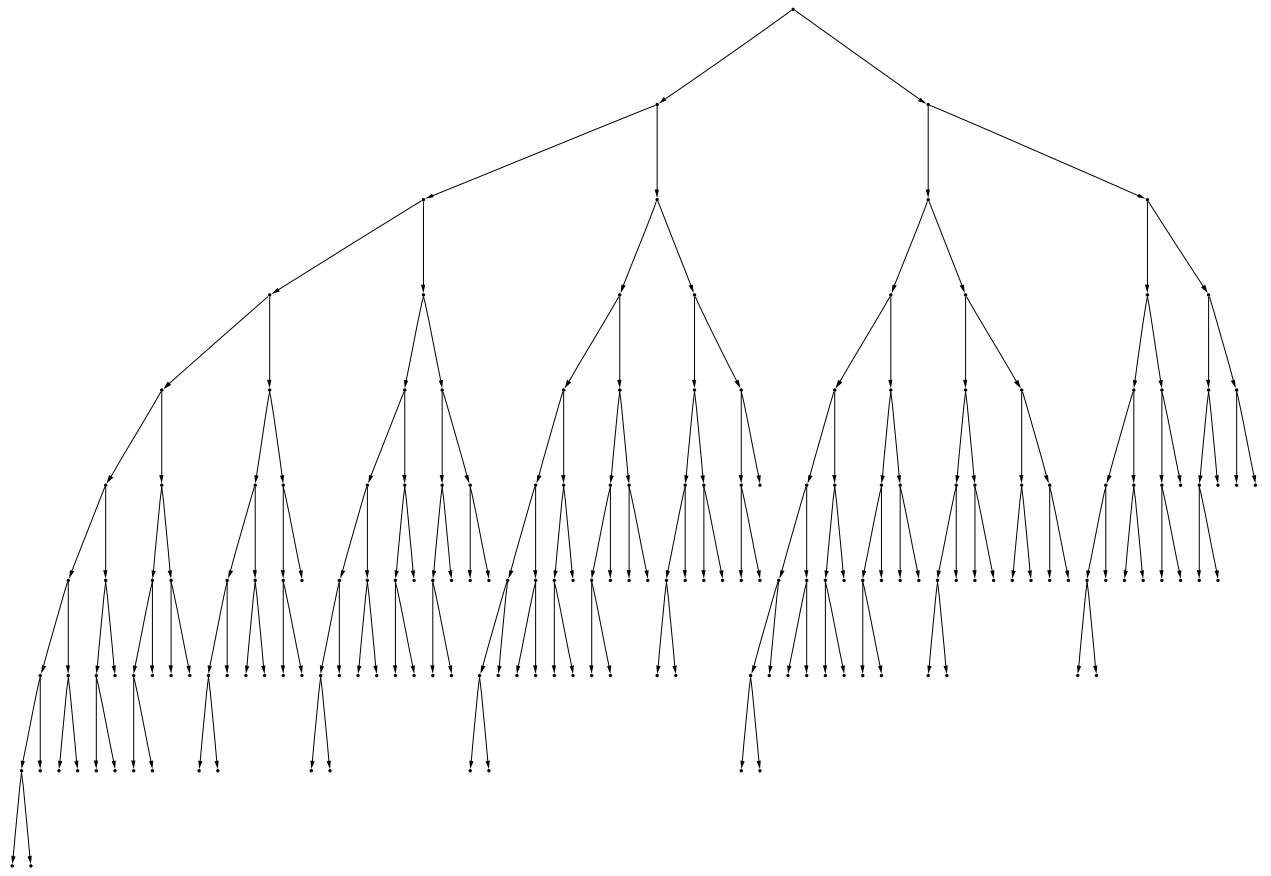

Figure 4.5: Parse tree created when $q=0.40$, parse tree strings are limited to 10 bits max and code lengths limited to 7 bits max. 


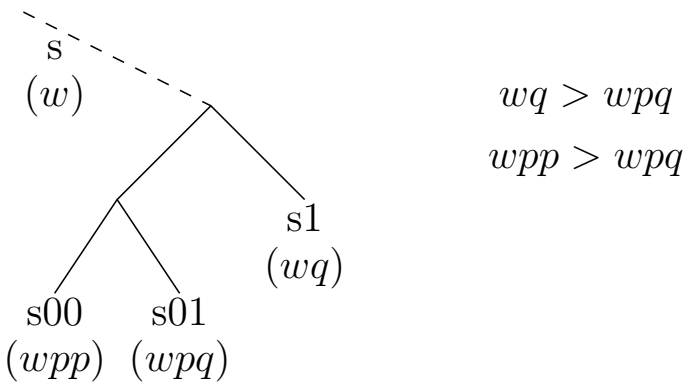

Figure 4.6: Illustration of proof of why the greedy merging process always selects a leaf that is part of a pair (i.e. has a sibling). A 0 corresponds to a left branch.

value after the merging step, the value of $x^{\prime}$ is:

$$
x^{\prime}=x-\left|s_{1}\right|(w p+w q)+\left(\left|s_{1}\right|-1\right) \times \frac{w q}{q} .
$$

Since $w q / q=w$ and $(w p+w q)=w(p+q)=w$, this equation becomes

$$
\begin{array}{r}
x^{\prime}=x-\left|s_{1}\right| w+\left|s_{1}\right| w-w \\
x^{\prime}=x-w
\end{array}
$$

In other words, at each merging step the value of the denominator decreases by the sum of the weights of the leaf and its sibling, which is equivalent to the weight of their parent node. From this we can prove that the greedy merging process causes the denominator of the coding rate equation to decrease the least, compared to other choices for merging.

Compared to other merging options, the greedy approach results in the smallest decrease in the parse tree's total weighted path length. In other words, the merging process selects leaves such that $w$ (as used in equation 4.4) is the smallest value possible.

The explanation is as follows: the greedy algorithm always selects the leaf with the smallest weight. We demonstrated earlier that this would always be a leaf with a sibling leaf, and that the selected leaf would always correspond to a string ending in a 1 .

Let $w_{1}$ be the weight of the path from the root to the parent of the leaf pair containing the least-probable leaf, as illustrated in figure 4.7. The weight of the selected leaf will be $w_{1} q$. Let $w_{2}$ be the weight of the path to the parent node of any other leaf 


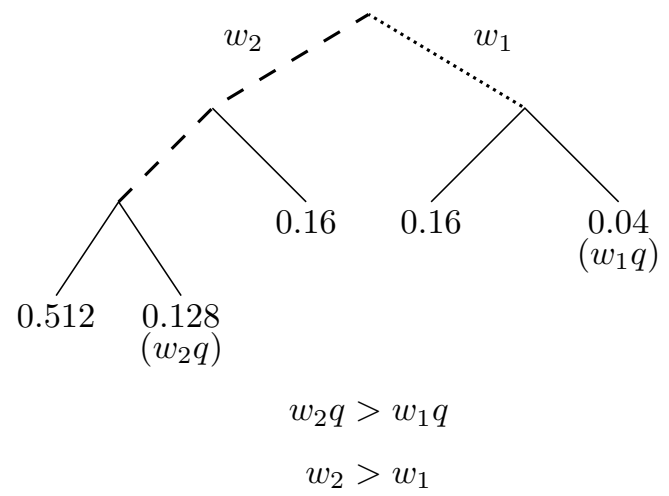

Figure 4.7: Illustration of proof of why the greedy merging process always causes the smallest decrease in the parse tree's total weighted path length. In this example $p=0.8$, $q=0.2$. A 0 corresponds to a left branch.

pair. The leaf with least weight in this pair will be $w_{2} q$. Since $w_{1} q$ is the least-probable leaf, we know that $w_{2} q>w_{1} q$. Dividing by $q$ we have $w_{2}>w_{1}$. The $w$ resulting from selecting the least-probable leaf and merging therefore must be the smallest ${ }^{1}$.

What does this property give us? Since the coding rate of a code is the ratio of the weighted sums of its code lengths and parse tree path lengths, keeping the denominator (the sum of the weighted parse tree path lengths) as large as possible is a reasonable step to take to ensure that the coding rate is as small as possible. It turns out that the reasoning behind the Tunstall-Huffman approach to VV codes is similar: the change in the denominator of equation 4.1 can be described in a manner similar to equation 4.4: $x^{\prime}=x+w$. It therefore makes sense to pick the leaf of greatest weight for extension, as this will result in the sum of weighted parse tree paths being as large as possible.

However, this similarity between the merging and extension processes indicates that the merging process may have the same problem as extension: a greedy merging process may not produce the best coding rate. It turns out that this is indeed the case. The proof is by counterexample. As illustrated in figure 4.8, if we select a block size of 3 and create a full parse tree, the greedy merging process will select the leaf corresponding to string 111, with a weight of 0.064 . The resulting code tree is given, and the sums of the weighted string and code lengths are given.

\footnotetext{
${ }^{1}$ From this we see that another way of viewing the merging process is that it selects the internal node of least weight and "prunes" its children, causing that node to become a leaf.
} 
Instead of using the greedy approach, if we select another leaf, say 011, with a weight of 0.096 , we get the parse tree shown in figure 4.9. The resulting code tree, with the sums of the weighted string and code lengths are also given. The greedy method produces a parse tree and a code with a coding rate of 0.98 . The alternative, non-greedy method produces a parse tree and a code with a coding rate of 0.9797. Although these coding rates are extremely close, the non-greedy method has the better coding rate (no rounding was performed in obtaining these numbers).

The best that one can do, then, is to use an intelligent method to define a limited search space and from that space select the most optimal VV code that occurs, with the goal of minimizing the search time while retaining a high probability of obtaining a VV code with good compression efficiency. A greedy merging process is such a method. It it not guaranteed to find the optimal code, but it places some natural and reasonable bounds on the search space, and the resulting codes are very good, as illustated in figure 4.3.

\subsection{Properties of Merged VV Codes}

We use the Huffman algorithm [27] to generate codes for our parse trees. Given a set of inputs, the Huffman algorithm is known to produce an optimal code. For the more general case where BV Huffman is used for parse tree coding, we know, given $B, p$, and $q$ that the resulting Huffman code is optimal. We do not know if that parse tree and set of codewords yields a code with the best possible coding rate for that particular $p, q$-it may be that some value $b<B$ may give a more optimal code. The only way to tell is to try all possibilities $0<b \leq B$. This may seem like a lot of work, but if $B$ is a reasonable size (say, $\leq 16)$ the search space is very small.

VV codes are not as pleasant. Again, given a parse tree we know, for that parse tree, that the Huffman code is optimal. But, we do not know if that parse tree and set of codewords yields a code with the best possible coding rate for that particular $p, q$. With VV codes created by the merging process, each time the parse tree changes at each merge step, the code tree changes as well, and it is not easy to quantify exactly what the change in the code tree will be (we do not know how the code lengths will change without 

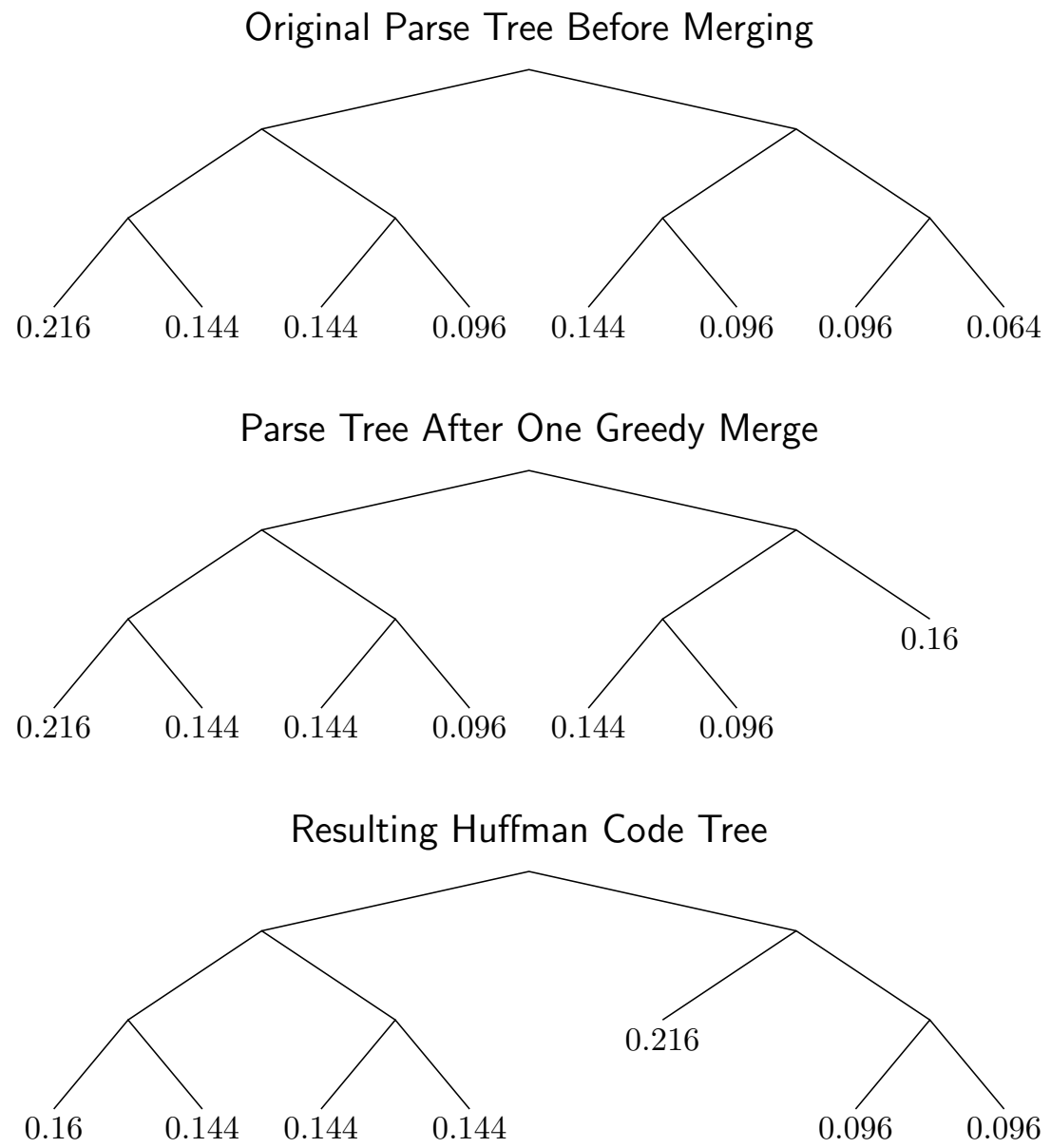

Sum of Weighted Parse Tree Path Lengths: 2.84

Sum of weighted code lengths: 2.784

Coding Rate: $\frac{2.784}{2.84}=0.9803$

Figure 4.8: Illustration of how the greedy merging process does not always result in the smallest coding rate. Results for a greedy merge. In this example $p=0.6, q=0.4$. A 0 corresponds to a left branch. 


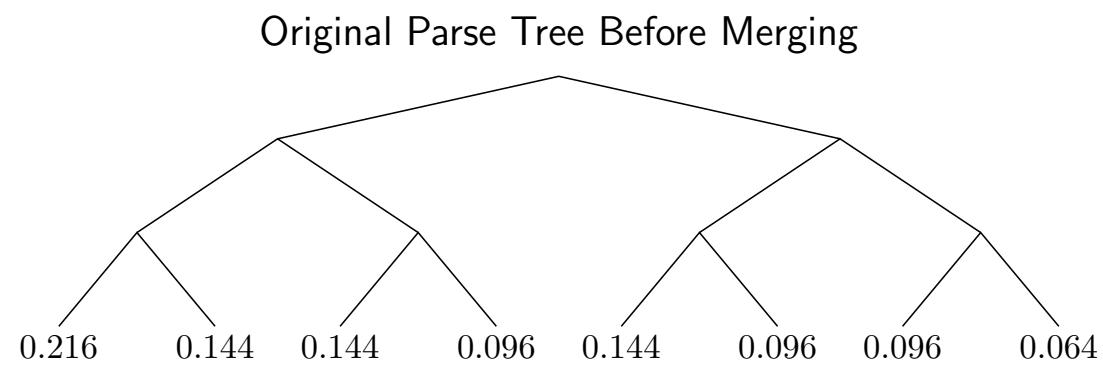

Parse Tree After One Non-Greedy Merge
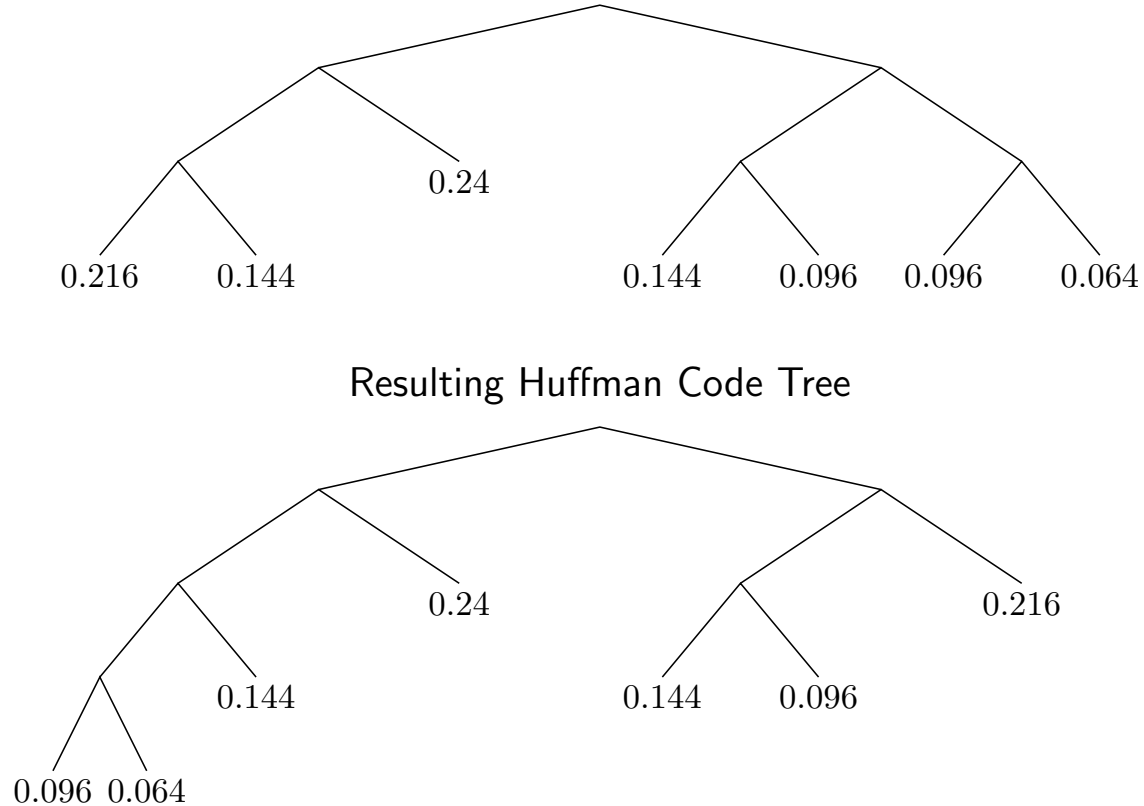

Sum of Weighted Parse Tree Path Lengths: 2.76

Sum of weighted code lengths: 2.704

Coding Rate: $\frac{2.704}{2.76}=0.9797$

Figure 4.9: Illustration of how the greedy merging process does not always result in the smallest coding rate. Results for an alternative merge. In this example $p=0.6, q=0.4$. A 0 corresponds to a left branch. 
actually re-generating them). Therefore we do not know, at each step, what the change in the overall coding rate will be -it may go up or down.

The only way to know is to try all possibilities, and indeed the only known method for finding an optimal VV code is through an exhaustive search. Unlike the BV codes, the search space for the VV codes is more vast as there are a variety of ways to form the parse trees, and for each of these trees the Huffman code lengths must be computed. Huffman code adaptation methods $[54,55]$ cannot be used to make the situation easier, because these methods assume that only the probabilities of the inputs change, and that the inputs themselves remain unchanged. This is not the case with VV codes, where one change to the parse tree results in a different set of inputs and a possibly very different Huffman code.

Figures 4.10 through 4.13 show the theoretical coding rate of VV codes created through merging, where the starting string lengths are 7, 8, 9, and 10. The merging process uses a greedy approach, selecting the least-probable leaf. The process terminates when the longest code length is the same as the starting string length. In each of these figures the $(W, C)$ pair for each trace indicates that the associated code has a starting string length of $W$ bits, and the longest allowable code length is $C$ bits.

From these figures we see several properties. The first is that it appears that if the starting string length is longer the coding rate is more resilient to changes. For example, the $(10,10)$ coding rate shown in figure 4.10 remains largely constant, changing from 0.476691 to 0.476732 as the longest code length changes from 30 to 15 .

The second is that at some point in the merging process the coding rate will change significantly, but it is not entirely obvious what causes a change to occur. Again referring to the $(10,10)$ code in figure 4.10 , at the 639th merging step the longest code length changes from 15 to 14, and at that point the coding rate begins to more noticeably degrade, in a linear fashion. Later at the 873rd merging step (the sharp peak in the coding rate) the coding rate begins to improve, but the longest code length remains unchanged at 12. This brings us to the next property of VV codes: the direction of change in the coding rate is unpredictable. Not only are local changes unpredictable, but when merging 
Coding Rate of (W,C) Codes, $\mathrm{q}=0.10$

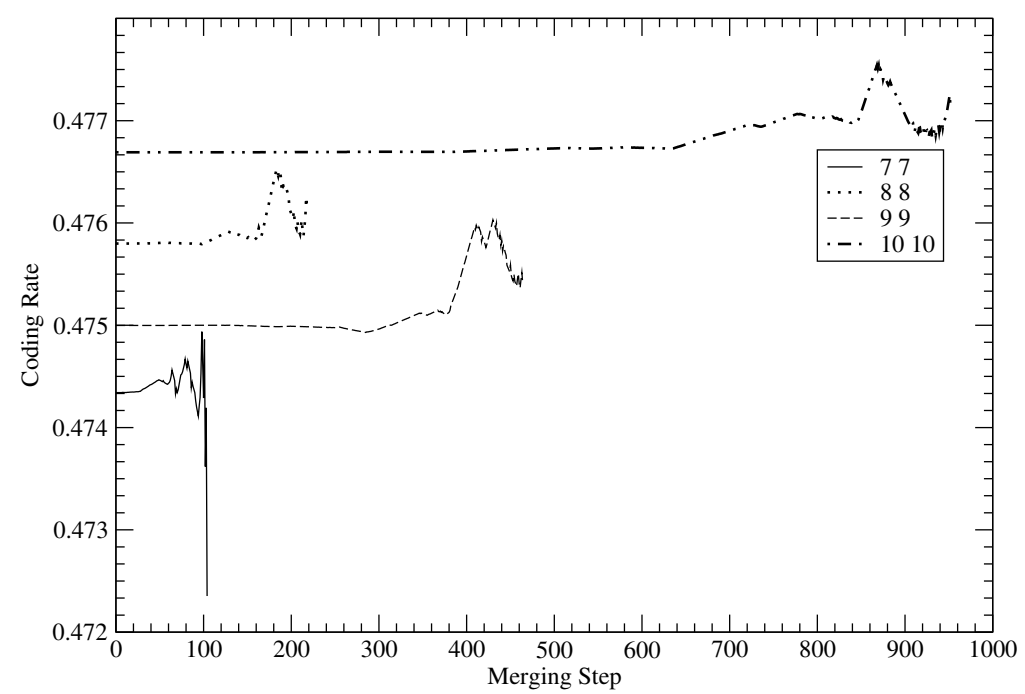

Figure 4.10: Coding Rate when $q=0.10$.

Coding Rate of $(\mathrm{W}, \mathrm{C})$ Codes, $\mathrm{q}=0.20$

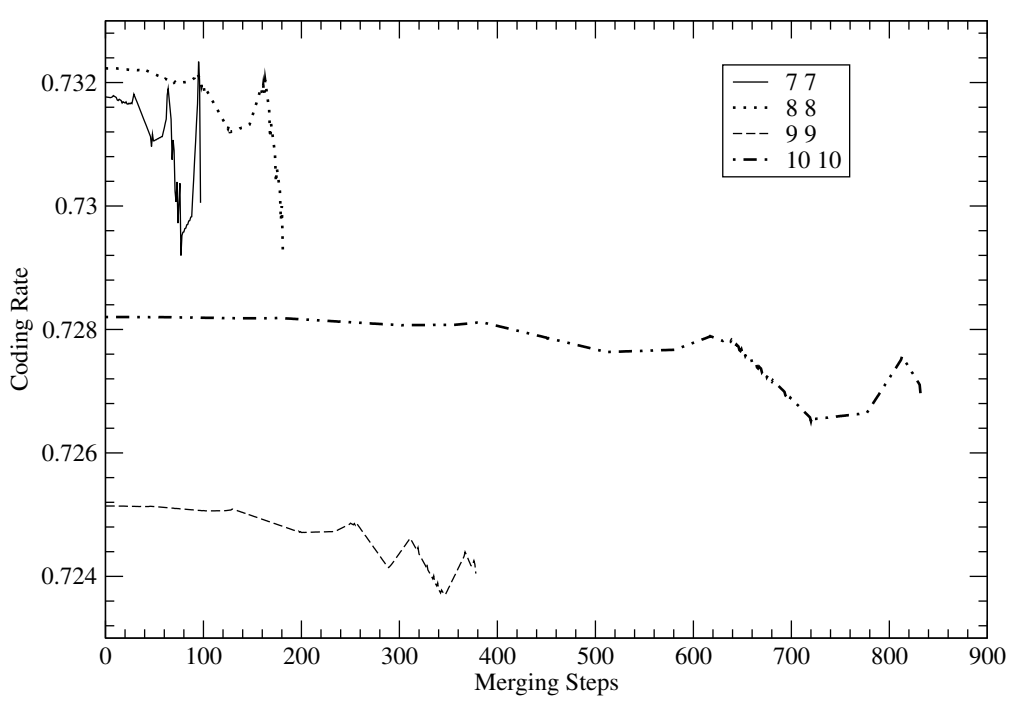

Figure 4.11: Coding Rate when $q=0.20$. 
Coding Rate of $(\mathrm{W}, \mathrm{C})$ Codes, $\mathrm{q}=0.35$

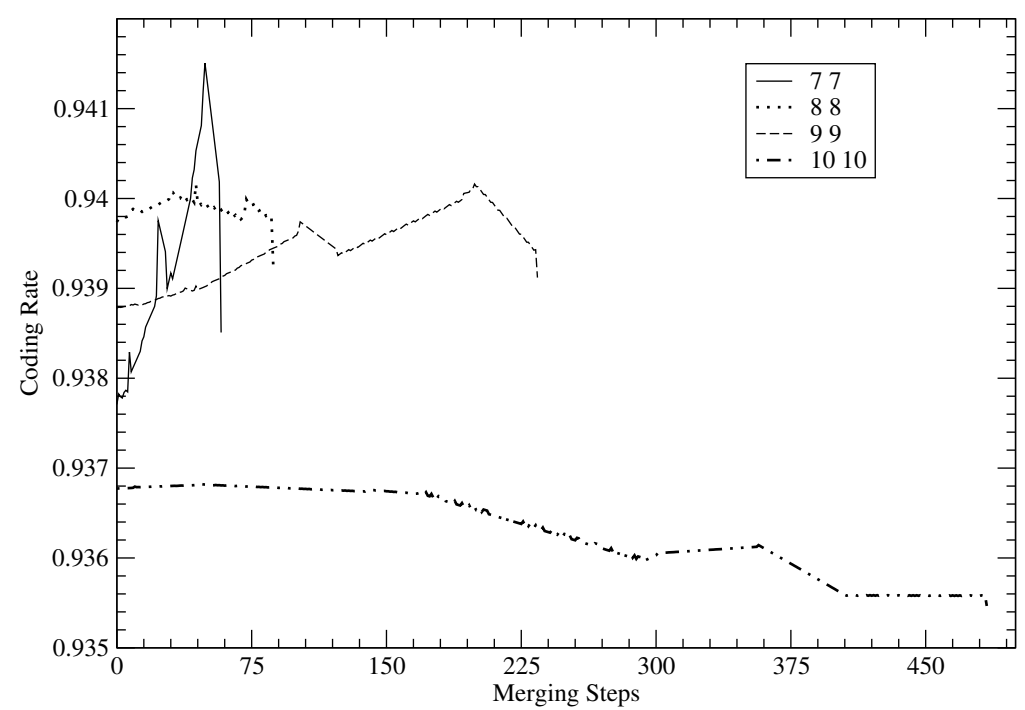

Figure 4.12: Coding Rate when $q=0.35$.

Coding Rate of (W,C) Codes, $\mathrm{q}=0.45$

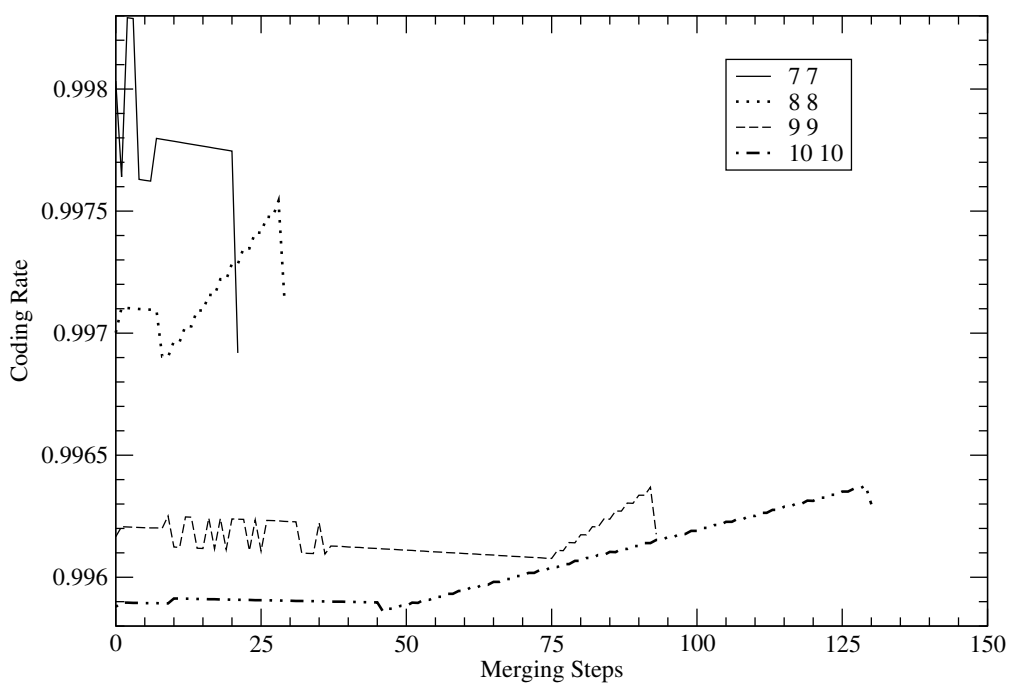

Figure 4.13: Coding Rate when $q=0.45$. 
terminates the resulting VV code will be more or less efficient than the starting BV code, but whether the final code is more or less efficient does not appear to be predictable.

Despite all this, these figures also show why VV codes are desirable for lengthlimited binary encoding: at the termination of the merging process the final coding rate is often not far removed from the pre-merging coding rate. For example, in the case of the $(10,10)$ code in figure 4.10 the final coding rate is 0.477102 , compared to an original rate of 0.476691 . In figure 4.12 the $(8,8)$ code's rate drops to 0.939259 from a starting rate of 0.939739 .

\subsubsection{Using VV Codes in Data Compression}

The inherent unpredictability of VV codes makes them extremely difficult to work with. Certain types of codes, such as BV Huffman, Golomb, and arithmetic, can be adapted to follow and more efficiently encode changing source probabilities. With VV codes this adaptation is generally not practical [49]. If VV codes are used to encode a source where the bit probabilities are changing, the only alternative is to have a collection of various VV codes on hand, and either swap between then on the fly, or use a bin coder, which is a collection of coders of memoryless sources. Swapping tables on the fly is appealing, but such a coder is not able to track changes on a bitwise basis. Once a table has been selected it must be used to process all incoming bits until a codeword is generated. We take the latter approach, creating a bin coder.

\subsection{Bin Coding}

The idea behind a bin coder is a simple one. We showed in section 2.3 that any source can be modeled as an interleaving of any number of memoryless sources. The probability modeler's task is to sort the source into several memoryless sources such that each source can be encoded with a code that is most optimal for that subsource's entropy. A bin coder is a collection of zero-order entropy coders, each coder being called a bin. Each bin is designed to efficiently encode a memoryless source over a given probability range. All the bins, taken collectively, cover the full range $0.5 \leq p \leq 1.0$. 
A bin coder diagram is given in figure 4.14. During the coding process, when a bit of input is read, the probability modeler determines which bin the bit should be placed into. When a bin accumulates a sufficient number of input bits, it outputs a codeword. These codewords are usually interleaved together into a single output stream.

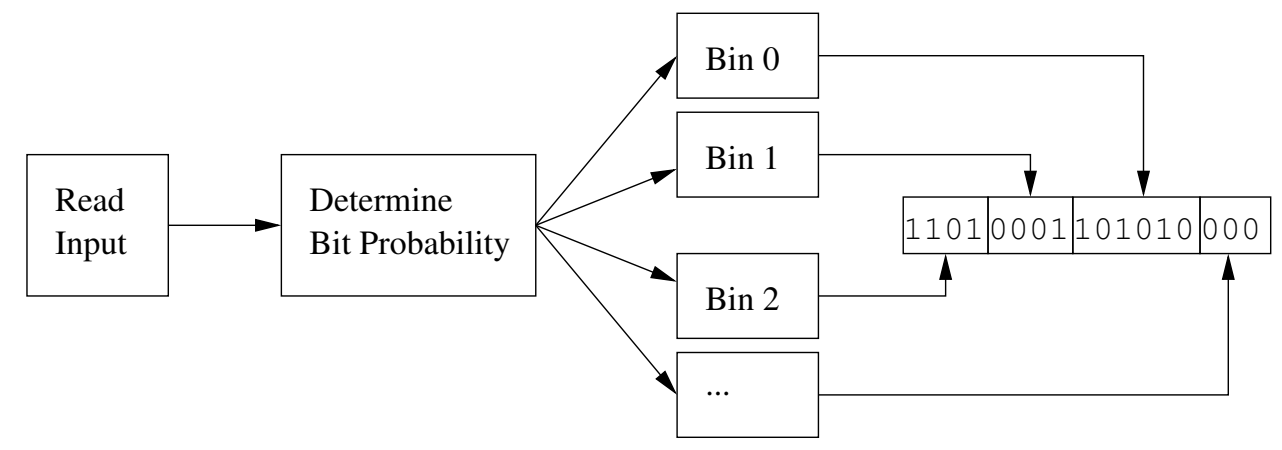

Figure 4.14: Diagram of a simple bin coder.

\subsubsection{Bin Output Interleaving}

One area of concern when using a bin coder is the handling of bin outputs. Unlike a single coder, in a bin coder each bin has its own output. How these outputs are handled has a significant effect on the coder's speed and performance. Usually it is desirable to have a single output from a data compression process. This means that when using a bin coder each bin's output must be interleaved into a single output stream. Since the encoding process is deterministic, the decoding process must also be, and this means that decoded bits must come out of the bins in the same order that they were encoded. This necessitates maintaining a strict bin priority ordering system where bins are given priorities based on the order in which their bits appeared in the source. A bin with a larger priority number is not allowed to place a codeword onto the output stream if a bin with a smaller priority number has not yet done so. If a smaller priority bin has not done so it is because it has not accumulated enough source bits to generate a codeword. In this circumstance extra empty bits ("flush bits") must be inserted into the bin to force an output. For a more detailed description of the interleaving, prioritizing, and flushing process, see [35]. 
This process of maintaining priorities and flushing bins does allow bin outputs to be successfully interleaved into a single output stream, but it also adds overhead to the encoding process, slowing it down and reducing the overall coding efficiency. If the interleaving is done on a coarser scale, one other than a codeword-by-codeword basis, it is possible to reduce the overhead but not eliminate it. Because of this overhead hurting performance, bin coders are not often favored when compared to other methods.

This type of fine-grained, codeword-by-codeword interleaving is appropriate for circumstances such as streaming live data over a single channel, or compressing an image on a desktop PC. However, there are circumstances in which interleaving may not be desirable. This might be the case when there is more than one output channel. Also, practically, a single object does not have to be comprised of one thing ${ }^{2}$. It can be comprised of many separate things, and as long as this is hidden from the user (or the user does not care) this is perfectly acceptable.

In our case, that of high-performance computing, our greatest concern is getting data encoded and written to disk as fast as possible so that the simulation can continue execution with minimum delay. We can use the extreme case: bin outputs do not need to be interleaved at all. This results in a total elimination of binning overhead and a corresponding increase in compression speed and efficiency.

\subsection{A Hybrid Bin Coder Utilizing VV Codes}

\subsubsection{Related Work}

Our work is related to the State-Tree Code (STC) adaptive VV coding method proposed by Stubley in [49], but differs in some respects. The STC's codes are generated from a set of parse trees with a specified number of leaves. The codes used are optimum - generated through an exhaustive search - and the number of strings in the code is necessarily kept small (8- and 16-leaf parse trees are used). Our codes are optimal within a limited search space, generated via the merging process (thus our search is technically not "exhaustive"),

\footnotetext{
${ }^{2}$ An example is the "application" in MacOS X, where a single application is actually a directory structure (navigable from the command line) containing code, resources, etc.
} 
and have a higher leaf count. We do not care how many leaves are in the parse tree, we only concern ourselves with the longest code length. Also, the STC does not divide up source bits according to probability, but only switches codes after parsing a binary string from the source. As the STC is not a bin coder, it does not have to address interleaving.

Boliek et al [8] at Ricoh created a high-performance bin coder. Their implementation is geared towards hardware (their results are taken from an FPGA hardware implementation) while ours is designed for implementation on a computer. They address the interleaving problem by interleaving code streams in fixed-length blocks, meaning that a particular codeword may be split between two blocks.

Kiely and Klimesh at JPL created an adaptable binary coder [29, 30] that also uses bins. Their technique differs from our method (and others) in that only the bin corresponding to $50 \%$ probability outputs a stream to storage. If the bins are ordered $q_{1}<q_{2}<q_{3}<\ldots<q_{n}$, with $q_{n}=0.50$, as the bits of the input stream are divided among the different bins and encoded, as each bin $q_{i}$ outputs a codeword, the bits of that codeword are redistributed to other bins $q_{j}, i<j$. The idea is that all bits work their way down to the $50 \%$ bin and are output. As of this writing, this coder is seeing use as the

back end for the ICER wavelet coder [31], which is being used by the Spirit and Odyssey Mars Rovers.

\subsubsection{Selecting VV Codes}

For this work we created a set of VV codes using greedy merging where the parse tree string lengths and code lengths were kept in the range $7 \leq W \leq 15$ and $7 \leq C \leq 13$. We created codes for $0.01 \leq q \leq 0.49$ at a granularity of 0.01 , and from them took the codes that had the least inefficiency. The curve resulting from these codes is given in figure 4.15. This figure shows that together the codes created in our work have an inefficiency well below $0.5 \%$, but that at about $q=0.04$ the inefficiency goes above $1 \%$. Our goal is to keep inefficiency below $1 \%$, so to encode bits that fall into $0<q \leq 0.04$ an alternative coding method is needed, and we choose Golomb coding. In this range the expected run lengths are such that optimizations can be made to quickly encode runs of bits in a single block 


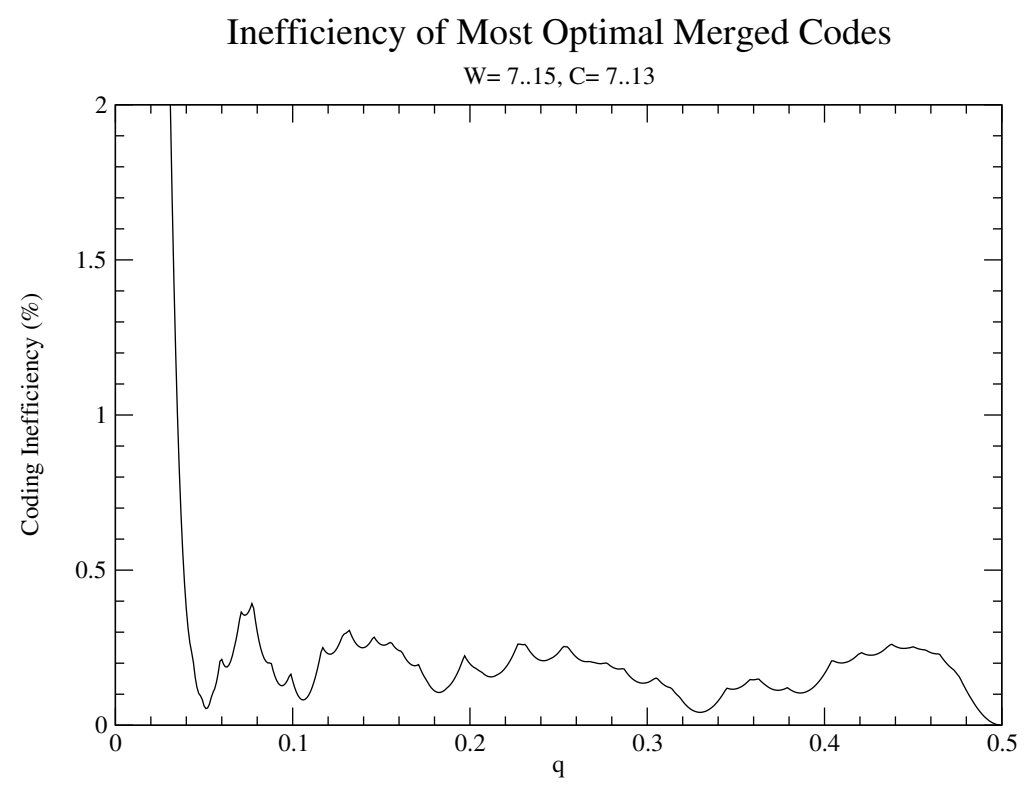

Figure 4.15: Inefficiency curve of the best merged VV codes.

and not bit-by-bit. We therefore propose a hybrid bin scheme: all bins below $q=0.04$ use Golomb coding, and all others use VV codes.

We created two hybrid bin coders, one with with 22 bins and the other with 25. In each case the coder used $18 \mathrm{VV}$ code bins, and the remaining were Golomb bins. VV codes used were selected from those created in our study. These coders perform no interleaving. A listing of the 22-bin coder details is given in table 4.1. The specifications for the bins used in the 25-bin coder are not available, as they were lost in a disk crash on an LLNL workstation that turned out to not be on the backup roster ${ }^{3}$. The process of selecting codes for the bins is currently manual, and done by visually inspecting the inefficiency curves and choosing codes that give us an inefficiency curve that falls below the limit we desire.

\footnotetext{
${ }^{3}$ Always make sure your files are being backed up! Do it yourself if you have to.
} 


\begin{tabular}{|c|c|c|c|c|}
\hline$\#$ & Bin Type & Code $q$ & $W$ & $C$ \\
\hline \hline 1 & VV & 0.49 & 7 & 7 \\
2 & VV & 0.44 & 13 & 13 \\
3 & VV & 0.38 & 13 & 12 \\
4 & VV & 0.33 & 12 & 13 \\
5 & VV & 0.32 & 12 & 12 \\
6 & VV & 0.30 & 15 & 12 \\
7 & VV & 0.27 & 13 & 12 \\
8 & VV & 0.24 & 15 & 13 \\
9 & VV & 0.20 & 15 & 13 \\
10 & VV & 0.18 & 13 & 12 \\
11 & VV & 0.16 & 15 & 10 \\
12 & VV & 0.14 & 15 & 10 \\
13 & VV & 0.10 & 11 & 10 \\
14 & VV & 0.11 & 12 & 11 \\
15 & VV & 0.01 & 7 & 7 \\
16 & VV & 0.10 & 10 & 8 \\
17 & VV & 0.05 & 11 & 9 \\
18 & VV & 0.05 & 13 & 9 \\
19 & Golomb & 0.04 & - & - \\
20 & Golomb & 0.03 & - & - \\
21 & Golomb & 0.005 & - & - \\
22 & Golomb & 0.002 & - & - \\
\hline
\end{tabular}

Table 4.1: Bins for the 22-bin coder. 


\subsubsection{Results}

We compared our bin coders to three other coders: the Augmented ELS-coder $[59]^{4}$, the Z-coder $[9]^{5}$, and an arithmetic coder written by us. The ELS-coder does not appear to have been designed with speed as a concern [60], but we include it because its coding rate is state-of-the-art. In this work we are interested in measuring and comparing the efficiencies of the coders themselves, not the efficiency of the probability estimation techniques developed for these coders - we supply the bits and the probabilities to be used to encode them. We therefore decoupled the the actual coders from their state tables and from each state table created a lookup table so that, given a bit and a probability, the coder could obtain the information it needed.

As we assume without loss of generality that the least probable bit is a 1, all our results are given with respect to $q$, the probability of encountering a 1 . We tested each coder on nonstationary sources, where $q$ varies bit-to-bit. To test for a nonstationary source we created a block of 256 probabilities, given according to the following equation:

$$
P(i)=\frac{i^{x}}{2 \times 256^{x}}, 0<i \leq 256
$$

where $1<x \leq 10.1$ is a floating-point parameter that determines the probability distribution, and $i$ is an integer. Some sample probability curves are given in figure 4.16. The resulting probabilities were converted to the form used by each coder. After a block was generated it was permuted, and a 30 megabyte bitstream was generated by repeatedly iterating through the block, generating one bit per entry based on the probability in that entry.

Timings were taken on a 1-GHz Intel Pentium III computer running Red Hat Linux. All execution times given are an average over 5 runs. Execution time for the bin coder is a total of time to both bin and encode all the source bits. Results for the execution times of the coders are given in figure 4.17. Time results for the 25-bin coder were similar to the 22-bin one, and are not shown. The "Exponent" axis values indicate the parameter

\footnotetext{
${ }^{4} \mathrm{ftp}: / /$ www.pegasustools.com/Osaugels.zip

${ }^{5}$ http://cvs.sourceforge.net/cgi-bin/viewcvs.cgi/djvu/djvulibre-3.5/libdjvu
} 


\section{Bit Probabiliry Distribution Curves}

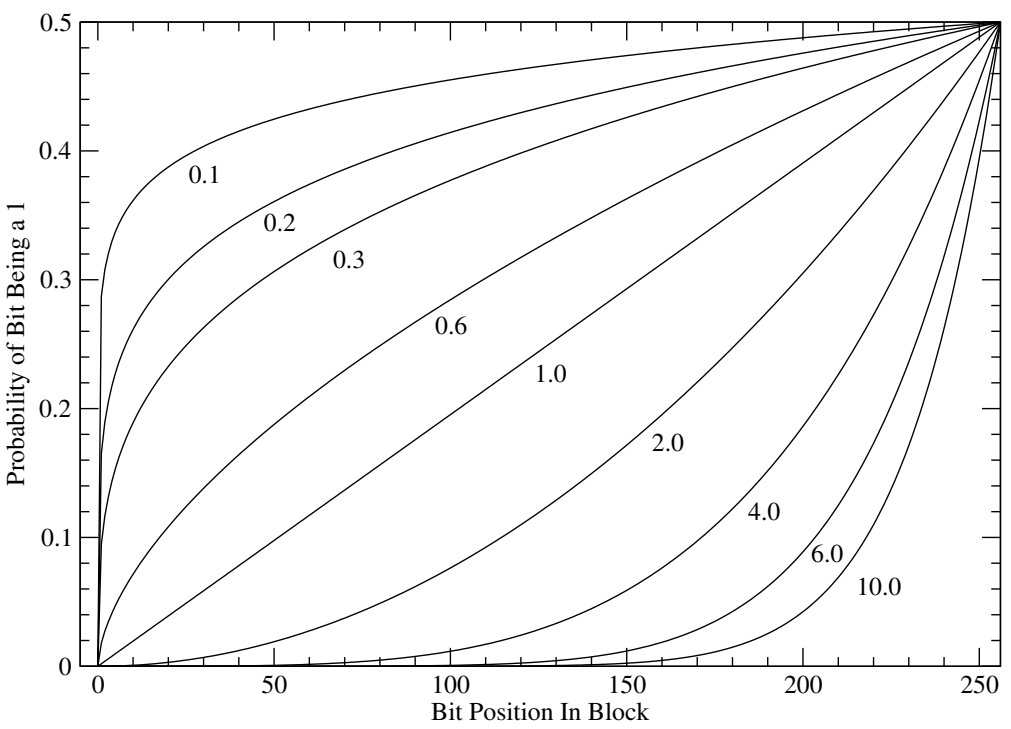

Figure 4.16: Some test probability distribution curves, computed according to equation 4.5. The number by each curve is $x$, and the bit position is $i$.

$x$, used to determine the bit distribution.

Figure 4.18 gives the coding inefficiency of each coder, as computed by equation 3.3. For more skewed probability distributions there is some experimental error, which is particularly apparent with the arithmetic coder in the form of its negative inefficiency. This is due to using an insufficient number of bits when taking the measurements at these skewed probabilities.

A more informative picture of the results is figures 4.19 and 4.20, comparing the ELS, Z, and Arithmetic coders respectively to the 22- and 25-bin hybrid coders. These figures give a graph of each coder's execution time versus its inefficiency. Smaller values are better, so an ideal coder would be one that is close to the origin, having a small inefficiency and fast execution time. In figure 4.20 we also show results for hybrid coders using 15-bit maximum length-limited BV codes, instead of VV codes. These BV codes are generated by the PM and Huffman algorithms, and are selected from the codes used to generate figure 4.2. The PM bin coder has 26 bins total, and the Huffman bin coder has 28, of which, respectively, 9 and 10 are Golomb code bins. 
Coder Execution Times

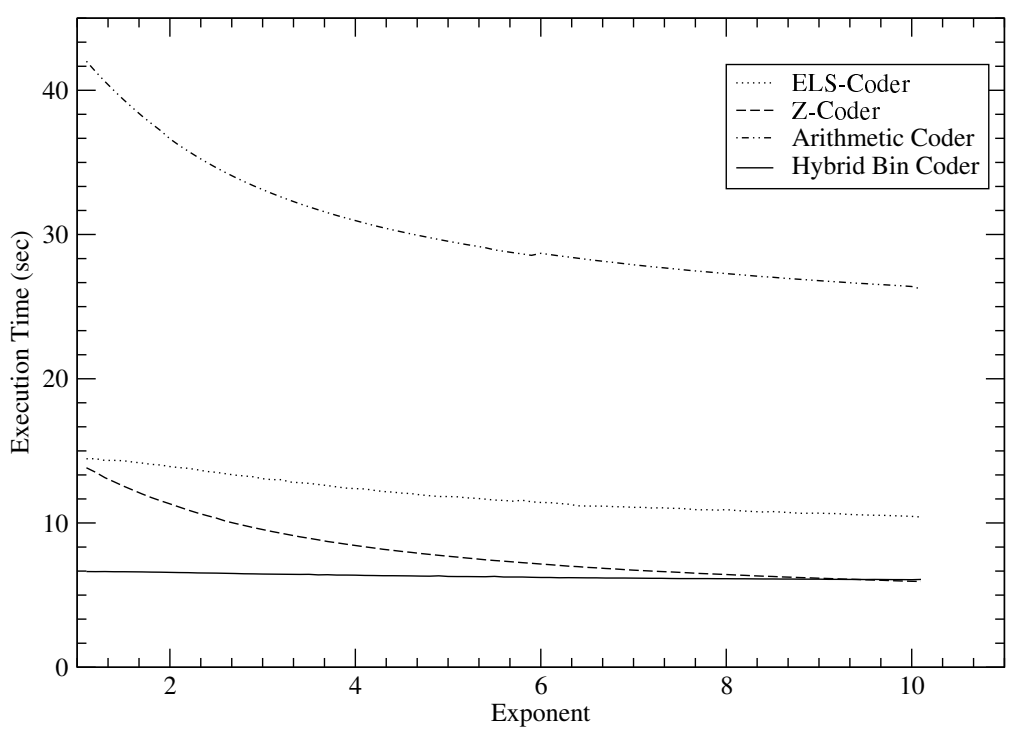

Figure 4.17: Execution time of the coders.

\section{Coding Inefficiency}

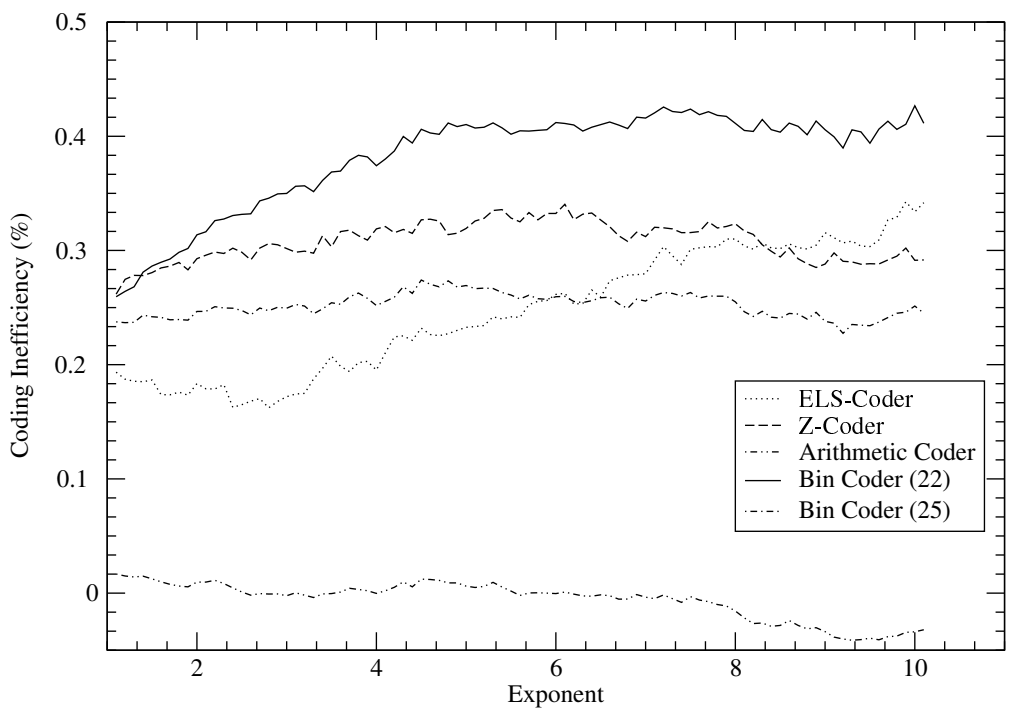

Figure 4.18: Coding Inefficiency of the five coders. 
Inefficiency vs. Encoding Time

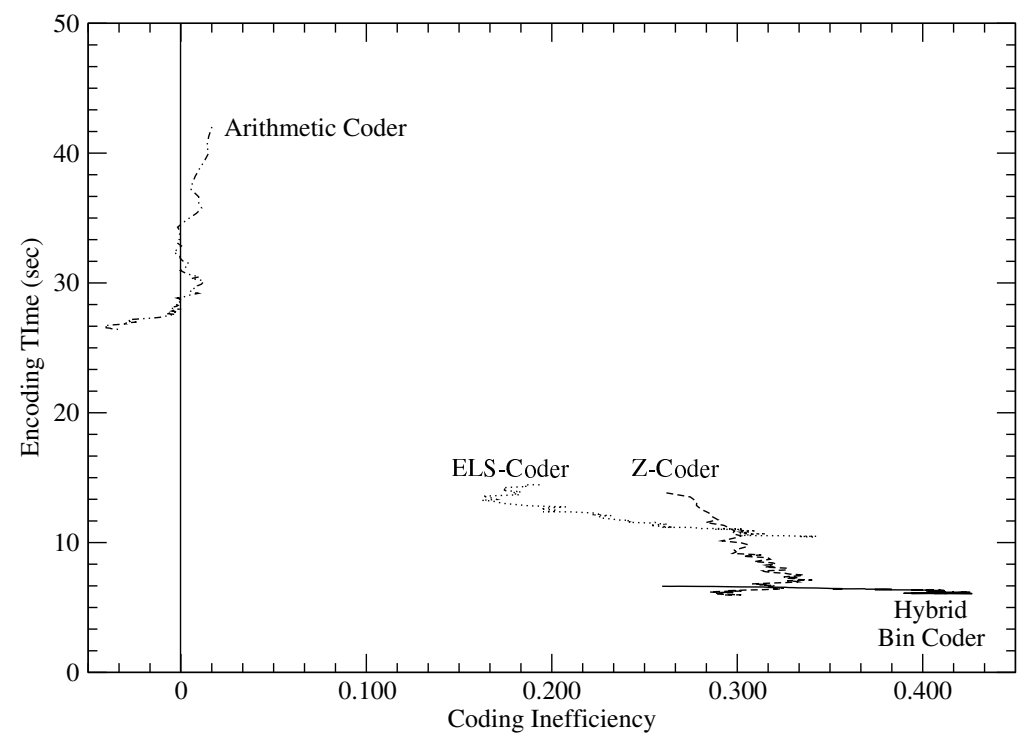

Figure 4.19: Coding Inefficiency vs. Time of the 22-bin hybrid bin coder.

Inefficiency vs. Encoding Time

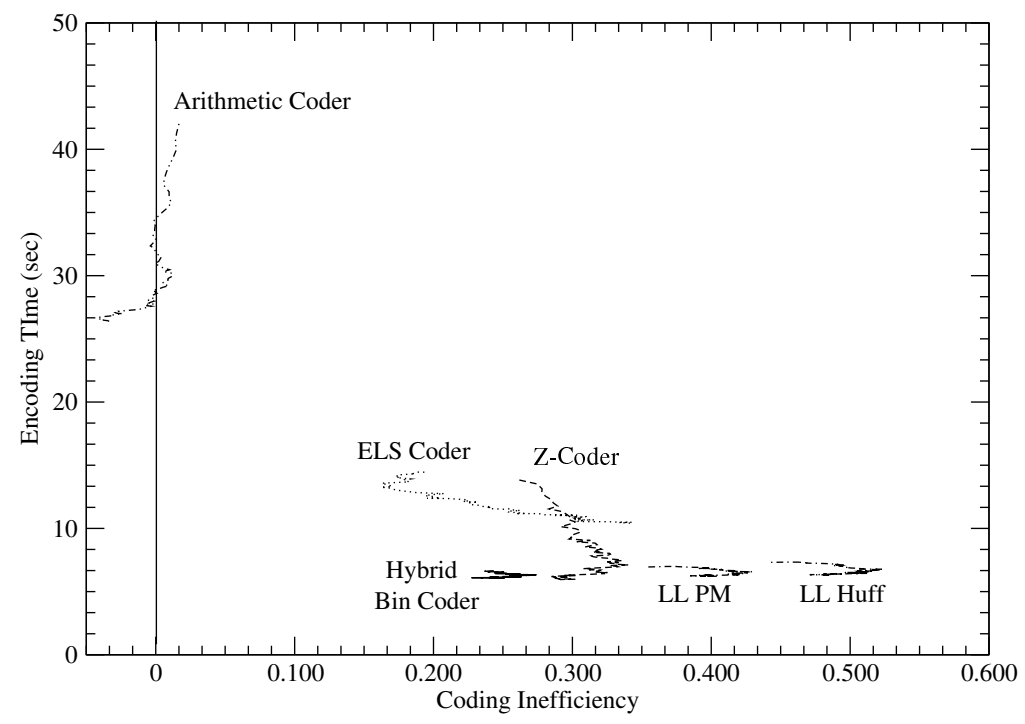

Figure 4.20: Coding Inefficiency vs. Time of the 25-bin hybrid bin coder. 


\subsection{Discussion}

From figure 4.17 we see that the Bin Coder's execution time is generally the same for all tested bit distributions. This is a plus-assuming that the incoming bits' probabilities are reasonably modeled the execution time is consistent. The execution times of the arithmetic, Z, and ELS coders vary as the bit distribution varies.

From figures 4.19 and 4.20 we see that the the addition of three more Golomb bins has reduced the inefficiency dramatically while leaving the execution time relatively unchanged. We also note from figure 4.18 that the 25 -bin coder's best-case inefficiency has improved from $0.27 \%$ to $0.23 \%$, when $x=1.1$. This indicates that proper handling of the extreme probability ranges is essential, even in cases where the percentage of bits that fall into the extreme ranges is the same as those that fall into other ranges.

We think that a hybrid bin coder holds promise for high-performance computing applications. The methods used in the bins (Golomb and VV coding) are fast, simple mechanisms, and the indications are that on a common architecture its performance rivals that of state-of-the-art coders.

\subsection{Future Work}

Bin and code selection in this study was by hand. It should be possible to automate this process, allowing the user to set a number of parameters such as memory requirements, number of bins, anticipated probability distribution, desired inefficiency level, etc., and have the computer make the bin selections.

The bin coders in this study do not perform output interleaving. A study into interleaving at fixed granularities (e.g. each bin must place $n$ codewords at a time on the output stream) should prove informative. Also, it may be possible to develop interleaving techniques for varying granularities. As an example, if bin 1 outputs codewords five times as often as bin 0 then it is inefficient to interleave outputs from both bins at the same,

fixed granularity. A system that requires bin 0 to place a single codeword on the output stream, but requires bin 1 to place 5 codewords, would likely be much more efficient. Such 
a system could even be made dynamic.

We use a greedy heuristic to select which leaf on the parse tree is selected for merging, and this works well. All other research into VV codes uses the extension method. Assuming that extension and merging stop as soon as the longest codeword is equal to some threshold, the parse trees created by each method will be different, and therefore the coding rates will be different. We need to establish how the merging method performs in comparison to the extension approach. 


\section{Chapter 5}

\section{Lead-1 Encoding and Surface Contouring}

In section 4.2 we reviewed encoding via table lookup, and discussed the problem of table size and ways of limiting it. Here we revisit the problem, and one solution, in the context of extracting isosurfaces from time-varying volume data. This work was done by Benjamin Gregorski and co-authored by myself. Project details appeared in [21], and some of the material here, such as the tables, most of the figures, and the results, is taken from it. Additional details are in [22]. Here we give the information related to the data compression method that was implemented for the project. The description is simplified somewhat; for complete details see the previously mentioned references.

\subsection{Volumes and Isosurfaces}

Three-dimensional volumetric data is often given as a three-dimensional grid (array), where each point in the grid has one or more numerical values associated with it. These values may be created by a process such as a numerical simulation, a scanning technique (such as MRI), etc. One method used to see, or visualize, this numerical data is to extract an isosurface from it. Given a value, called an isovalue, the cells of the 3D grid are scanned and polygons drawn in each cell containing the isovalue. Isosurfaces for two different data 

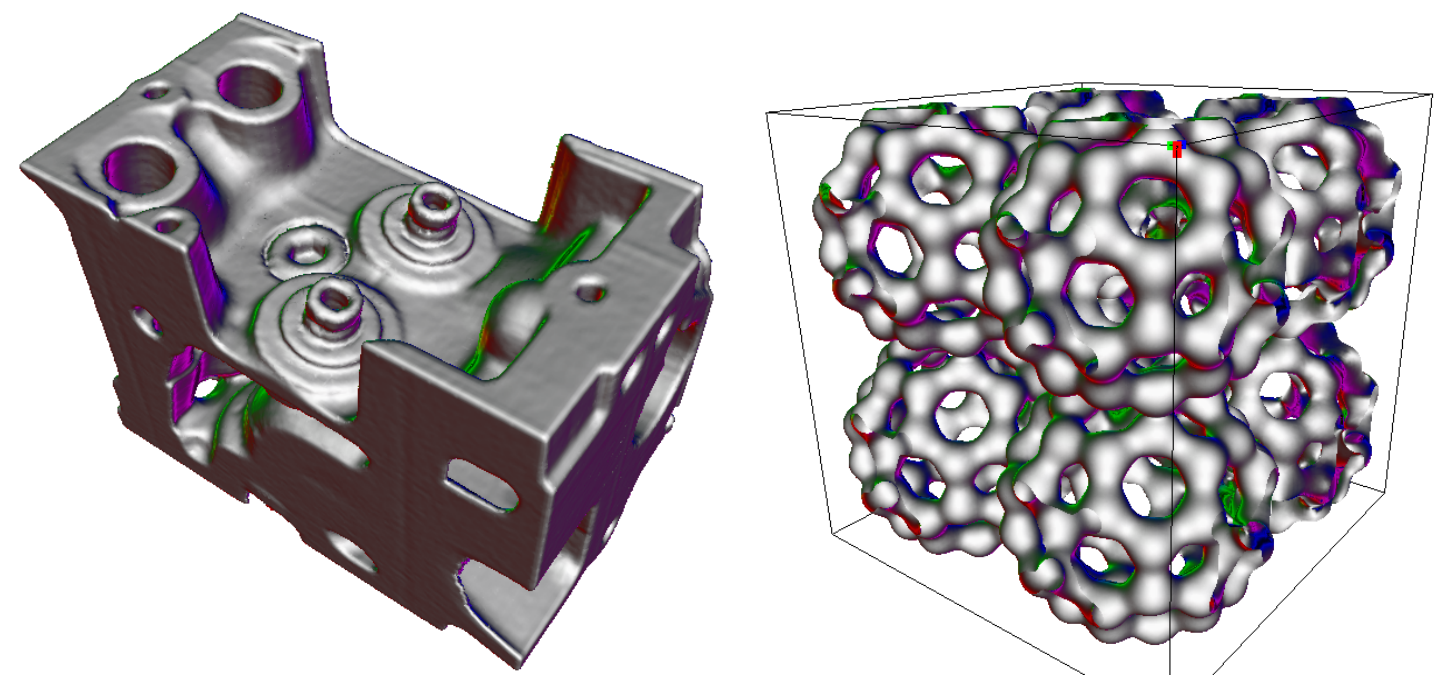

Figure 5.1: Two example isosurfaces. On the left is a surface of the engine block, on the right a surface of some Carbon "buckyball" molecules.

sets are given in figure 5.1.

\subsection{Time-varying Data and Contouring}

Given volumetric data that varies over time, we might want to extract a surface contour from this data and display it, seeing how the surface changes over time. In this circumstance all of the data is not likely to fit into memory, and even if it does the amount of data may make the data set unwieldy. In this case storing the data in compressed form is desirable. The data can be read in compressed form (resulting in faster loading), decompressed, processed, and the resulting surface displayed. To minimize any detrimental impact on the frame rate the data needs to be encoded using a method that allows for extremely fast decoding. Like in section 4.2 we chose a table-lookup method over other techniques, and again we ran into the problem of table size. Because of the amount of data being processed the decoder must be both fast and very small - it should fit in cache and not interfere with the other computations.

For data (such as time-varying data) that tends to vary smoothly a simple difference transform is often very effective in exposing redundancy for compression purposes. For example, the sequence $1,2,3,4,5,5,4,4,3,4$ will not compress very well, but if 
we leave the 1 unchanged, and then for each sequence number $i$ compute the difference between it and the the number in the previous position $i-1$ we have $1,1,1,1,1,0,-1$, $0,-1,1$. This sequence is a lot more redundant and will compress better. It is sometimes possible to get a lower entropy, or at least pull the transform coefficients toward zero, using double differencing: repeating the difference transform on the coefficients resulting from the first difference transform. For the time-varying data this is the approach we used, and the transform is stated formally in equation 5.2 .

$$
\begin{aligned}
& T_{C}(i)=D(i)-D(i-1), 0<i<n . \\
& T_{L}(i)=\left\{\begin{array}{r}
T_{C}(i) \quad: \quad i=1 \\
T_{C}(i)-T_{C}(i-1) \quad: \quad 1<i
\end{array}\right.
\end{aligned}
$$

$D(i)$ is an original data value. The first data value at $i=0$ is passed along unchanged. Since the data is time-varying we transform the data in the temporal domain, rather than the spatial. Each sequence of data values corresponds to the value of a single spatial location. For a dataset of dimension $256^{3}$ with 274 time steps a difference transform histogram is given in figure 5.2. From this we see that, although the range of the coefficient magnitudes has expanded, more of the coefficients are grouped around zero. The transformed coefficients will compress better.

\subsection{Lead-1 Encoding}

Given a set of transform coefficients, the histogram indicates that the values are at or clustered close to zero. If we examine the magnitudes of the coefficients (in binary), we see something like this: 00000000, 00000000, 0000010, 0000001, 0000010, 0000011, etc. In other words, because most of the coefficients are close to zero (or are zero) each coefficient is a string of zeros followed by a 1, and then maybe a bit or two. In these coefficients most of the redundancy is found in the position of the coefficient's leading 1. Table 5.1 gives lead -1 counts for the data set from section 5.2 , where 0 indicates a zero coefficient, 1 is the LSB, 2 is the next significant bit, etc. We see that after transformation most of 


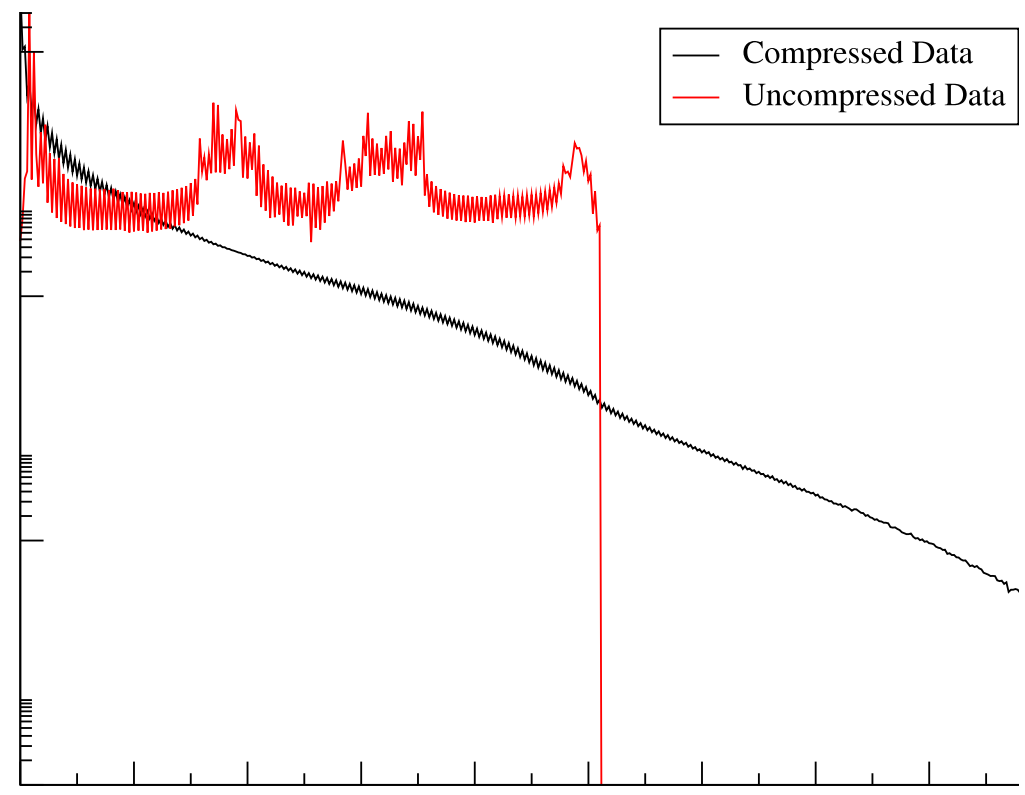

Figure 5.2: Histogram of coefficient magnitudes in a $256^{3}$ volume with 274 time steps. The vertical axis is on a logarithmic scale.

the leading-1 positions are zero, 1 , and 2 . As an example, in the coefficient 00000110 the leading 000001 compresses rather well, but the following 10 does not. Lead-1 encoding takes advantage of this observation, encoding for each coefficient the position of its leading 1, and passing all remaining bits (including the sign bit) on unchanged.

What effect does this have on the lookup tables? We saw earlier that when using the Huffman (or a similar) algorithm the worst-case code length is the number of input symbols $N$ minus 1 . For a 9 -bit magnitude coefficient, there are $2^{9}=512$ possible coefficients. While we're not likely to have a longest code length of 511 bits, it's very possible that a code length will be 16 bits or longer.

If we encode only the position of the leading 1, the number of symbols that need to be encoded is 9 , plus one for the zero coefficient. Thus the longest code length cannot go over 9 bits, and the decoding table is limited to a maximum size of $2^{9}=512$ entries. Each entry in the decoding table only needs to give the position of the leading 1, and since there are only 10 positions this information fits into a byte. The maximum possible size for the decoding table is 512 bytes. The table will easily fit into cache memory and is not likely to interfere with other computations and data. 


\begin{tabular}{|c|c|c|}
\hline Lead-1 & Untransformed & Transformed \\
\hline \hline 0 & 4743194 & 6093309314 \\
1 & 9297339 & 1075445689 \\
2 & 61897415 & 1453200739 \\
3 & 4119536406 & 1010756643 \\
4 & 408098714 & 880931513 \\
5 & 282209288 & 704350384 \\
6 & 412069938 & 479577928 \\
7 & 2753591178 & 251551499 \\
8 & 3977820400 & 78543126 \\
9 & 0 & 1597037 \\
\hline
\end{tabular}

Table 5.1: Lead -1 position counts for a $256^{3} \times 274$ dataset.

\subsection{Results}

The data for the tests consisted of a $256^{3}$ volume with 274 timesteps, and came from the Richtmeyer-Meshkov instability dataset from Lawrence Livermore National Laboratory [33]. Each point in the volumetric data has a numerical value and a gradient vector. The raw data was 12.844 gigabytes in total size, or about 48 megabytes per time step. The data compressed to a $54 \%$ of original size.

To test the process of extracting isosurfaces from compressed time-varying volume data two versions, one using compressed and one using uncompressed volumes, were implemented. The test system was a $2 \mathrm{GHz}$ Pentium 4 with $1 \mathrm{~GB}$ of main memory and GeForce Ti 4600 graphics card.

To measure Disk I/O the tests were run so that the amount of data loaded from disk was roughly the same, whether using compressed or uncompressed volumes. Tests were performed loading data in quantities equivalent to 10, 15, and 20 uncompressed timesteps. Figure 5.3 shows the total number of memory page faults over time when loading compressed and uncompressed data. As expected, when using compressed volumes there is less data read from disk at one time and the frequency of disk reads is less.

Figure 5.4 compares the actual frame rate when using compressed vs. uncompressed volumes. We see that during the initial frames of the animation the system using compressed data is faster, but the two systems very rapidly approach the same frame rate. 

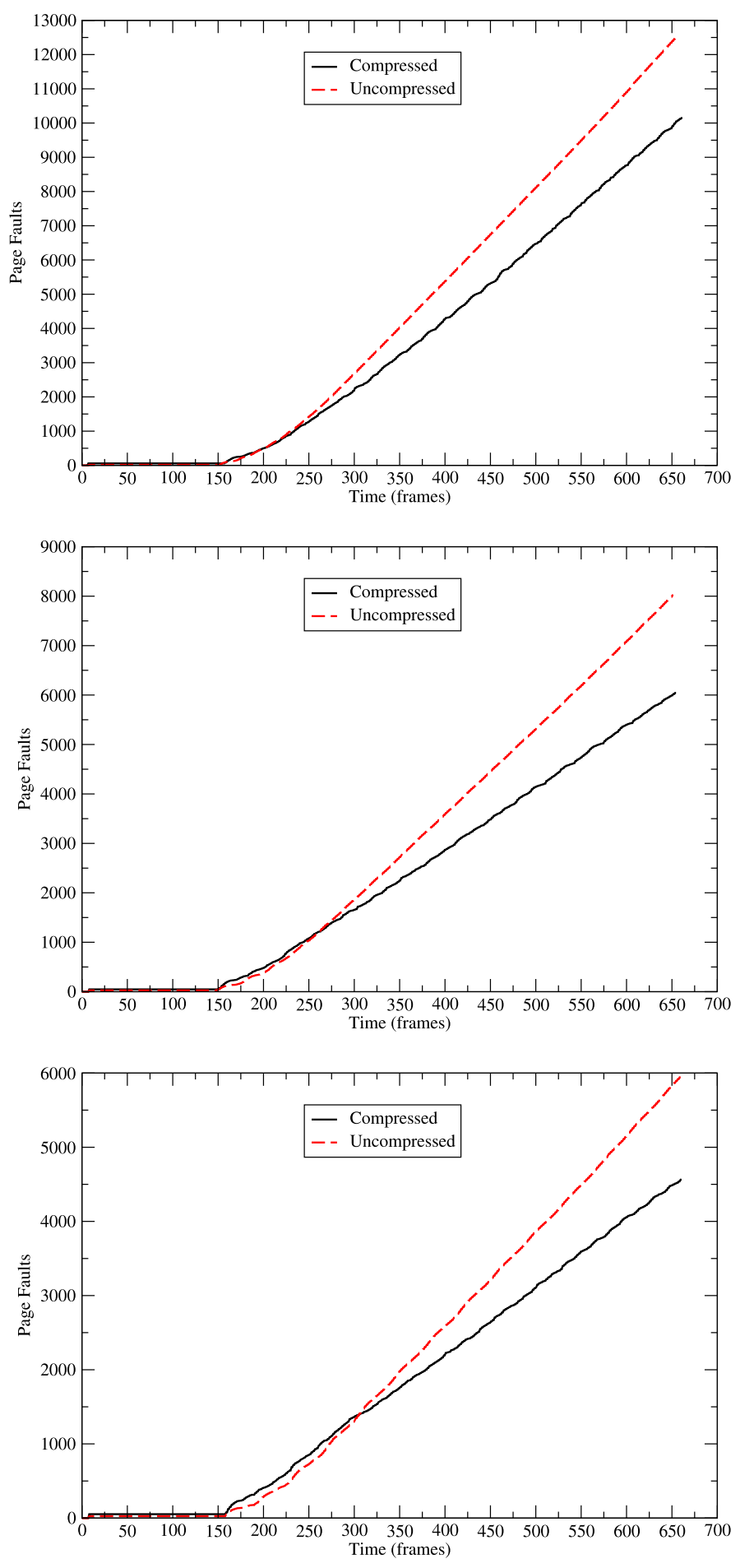

Figure 5.3: Page faults, in disk read sizes of (left to right) 10, 15, and 20 uncompressed time steps. 

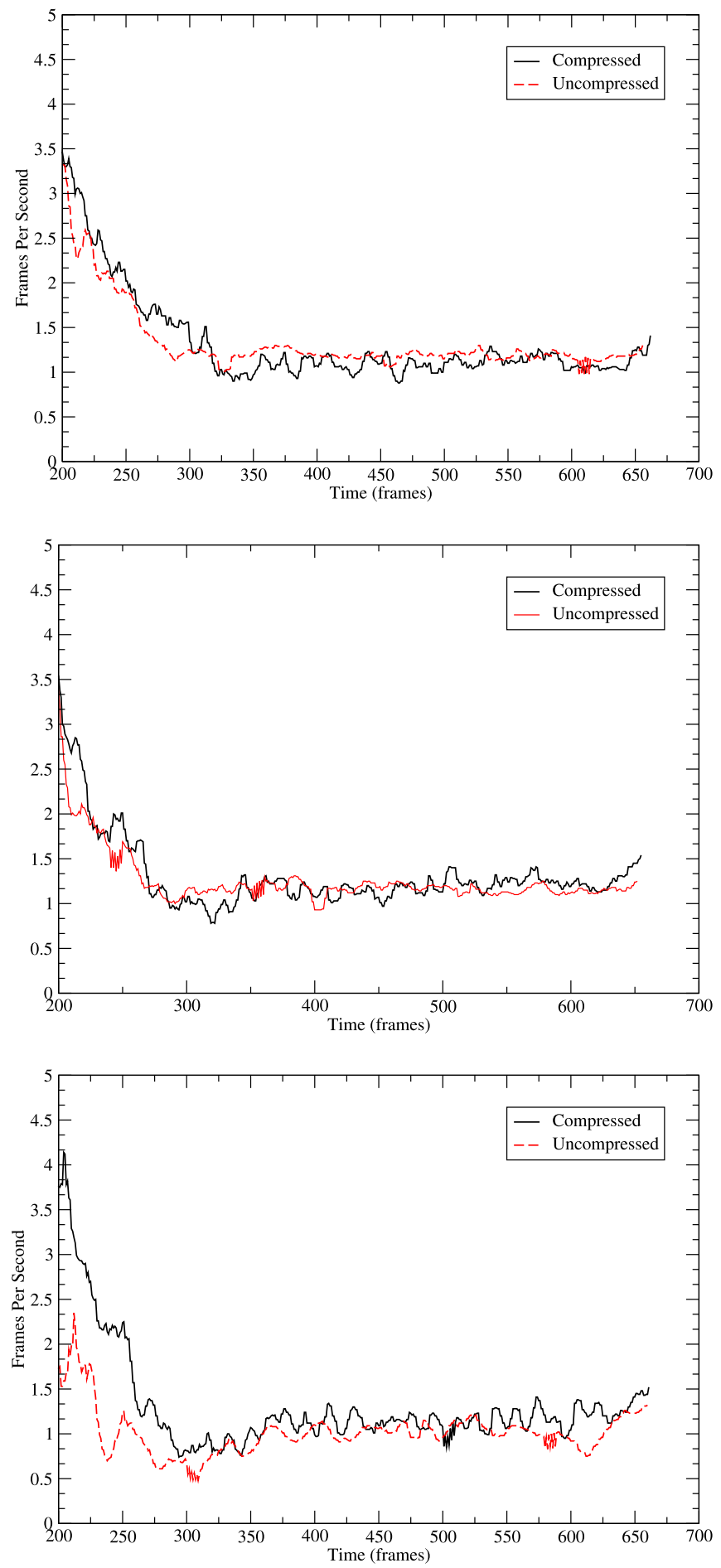

Figure 5.4: Frame rate, in disk read sizes of (left to right) 10, 15, and 20 uncompressed time steps. 
Although Lead -1 encoding is a fast process, it appears that in this case that the cost of decompressing the volume data results in a zero net gain over using uncompressed data. This lack of performance increase when using compressed volumes is unfortunate, but Lead-1 encoding still gives us a win in terms of resource use. We achieve an essentially identical frame rate while using significantly lower disk space. Also, if storage is remote as opposed to local (these tests were done with local storage) it is possible that the frame rate for compressed volumes would be higher, due to the lower latency of the disk reads.

\subsection{Conclusion}

Our use of Lead-1 encoding is an example of some of the trade-offs that exist, and decisions that need to be made when developing a data compression system. Lead-1 encoding, while not a state-of-the-art method in terms of compression efficiency, has the advantage of generating small (indeed, tiny) lookup tables and is a simple, fast method. If speed is of prime importance, Lead-1 encoding provides a fast, compact method that can deliver a reasonable compression rate. 


\section{Part II}

\section{Data Transformation}




\section{Chapter 6}

\section{Wavelets}

\subsection{Introduction}

As mentioned in chapter 1 , when some data is to be compressed a transformation is often performed on the data prior to its being encoded. The transformation decorrelates the data, which makes it easier to compress. A transform now very common in data compression (and other fields) is the wavelet transform. There are many different types of wavelets, most of which will not be reviewed here. Here we review the basic principles behind a wavelet transform.

So what exactly is a wavelet transform? The underlying principle of a wavelet transform is that a signal can be described as the sum of translations and scalings of a waveform. The actual waveform (or "wavelet") used to facilitate the signal decomposition is what differentiates the various wavelet transforms. During a signal decomposition, the wavelet transform separates the signal into a hierarchical set of subbands, with the data organized in order of increasing significance. Given a signal, each step in the decomposition creates (1) an coarse-level approximation or "average" of the original signal, and (2) a set of detail coefficients that, together with the coarse-level approximation, can be used

to reconstruct the original signal. There are quotes around the word average because the coarse level approximation is, in most cases, not a strict average. 


\subsection{The Haar Wavelet Transform}

The Haar Wavelet Transform (see [20] for a description) is probably the simplest of the wavelet transforms, and is illustrated in figure 6.1. Given a signal, such as an image, the Haar transform takes a pair of adjacent data values $(A, B)$. From them it computes two values: a High-Pass value $H$, and a Low-Pass value $L$. These are computed according to equations 6.1 and 6.2. $L$ is the aforementioned approximation, or "average" of $A$ and $B$. $H$ is the detail coefficient - it contains the detail information that was removed to create the coarser approximation $L$. Naturally, the closer $A$ and $B$ are to each other in value the closer $H$ will be to zero, and the closer $L$ will be to both $A$ and $B$.

$$
\begin{aligned}
& L=\frac{A+B}{\sqrt{2}} \\
& H=\frac{B-A}{\sqrt{2}}
\end{aligned}
$$

After all input pairs are processed the result is an approximation of the original data (all the $L$ coefficients) and the details that were removed in creating the approximation (all the $H$ coefficients). The transform can then be repeated on the $L$ values. For a multidimensional signal (such as an image) the transform can be performed once along the rows direction, and then once along the columns direction (the Haar decomposition of an actual image is illustrated in figure 6.2). The process then repeats on the $L L$ values.

\subsection{Advantages of Wavelets}

So, what does the Haar (or any wavelet transform) give us? The first benefit of a wavelet decomposition is data decorrelation. Many kinds of data tend to be rather smooth, or change gradually. That is, for any two adjacent data values the difference between them is likely to be small. Some examples of data like this are photographs, hourly tide measurements, temperature readings, etc. Because of this property, after transformation the $H$ coefficients tend to be at or near zero. 


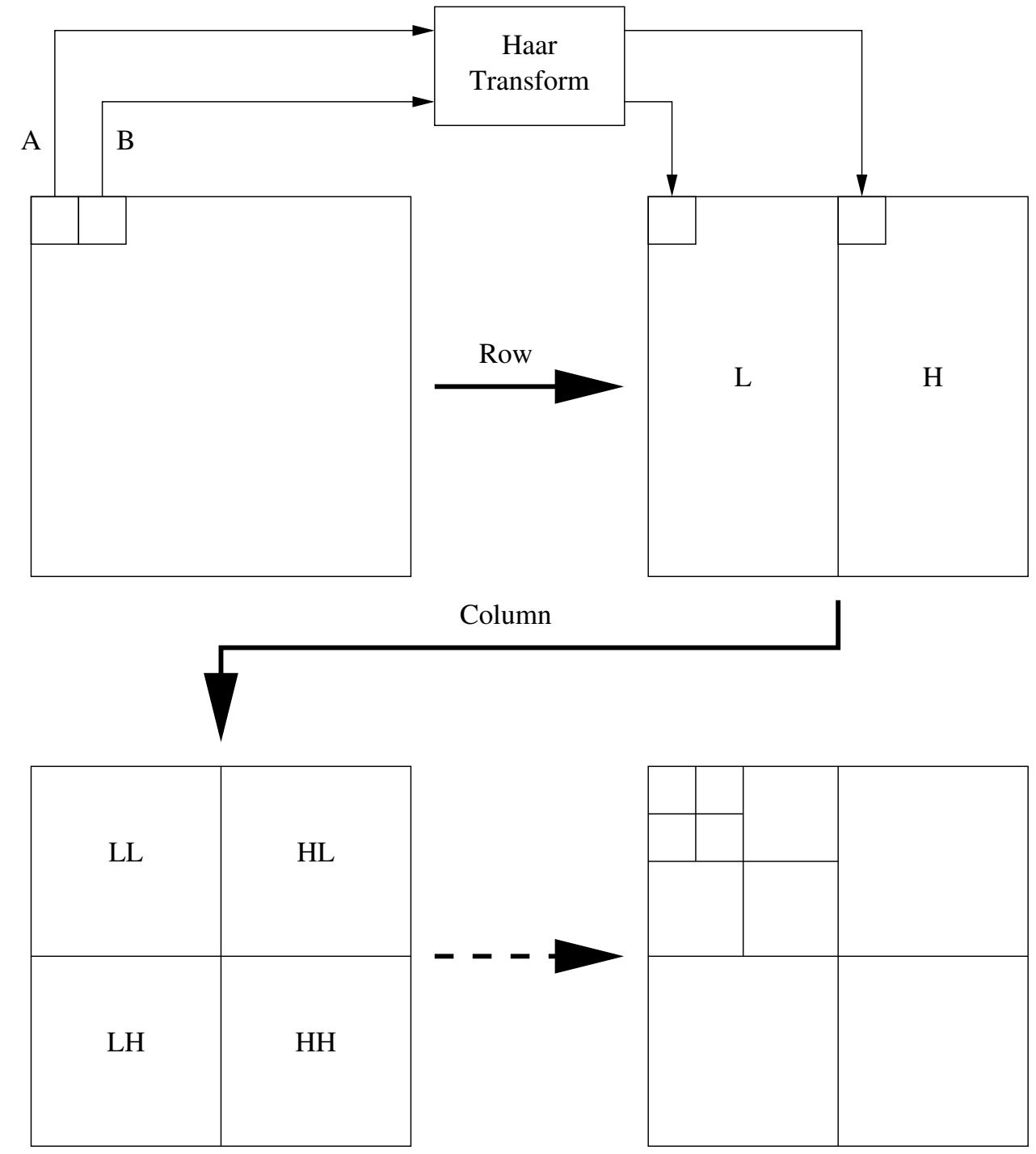

Figure 6.1: Haar Wavelet Decomposition. 

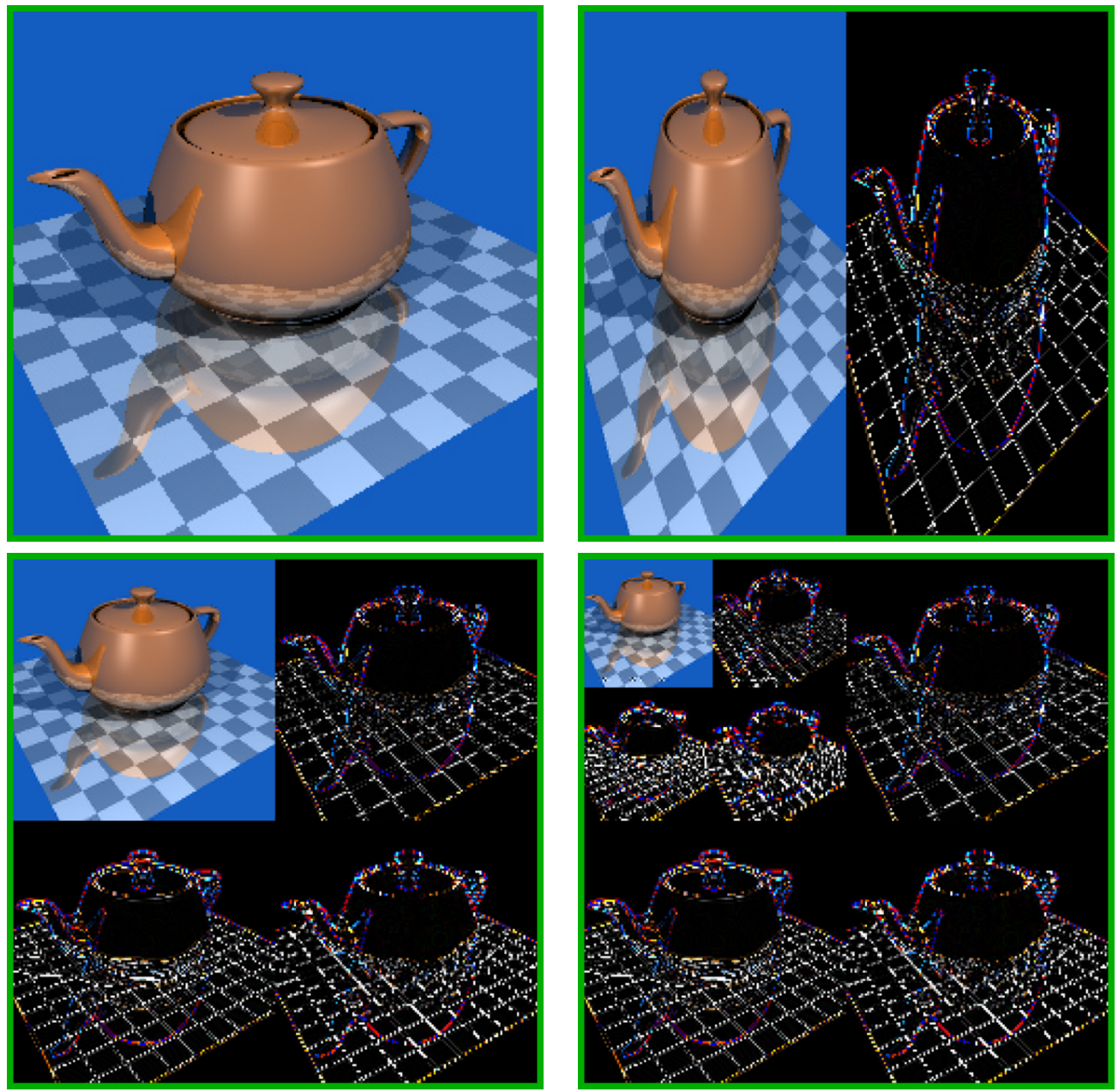

Figure 6.2: Haar Wavelet decomposition of an image. Images are from the 1996 SIGGRAPH course "Wavelets in Computer Graphics", edited by Wim Sweldens and Peter Schröder. 
Histogram Of Lena Image

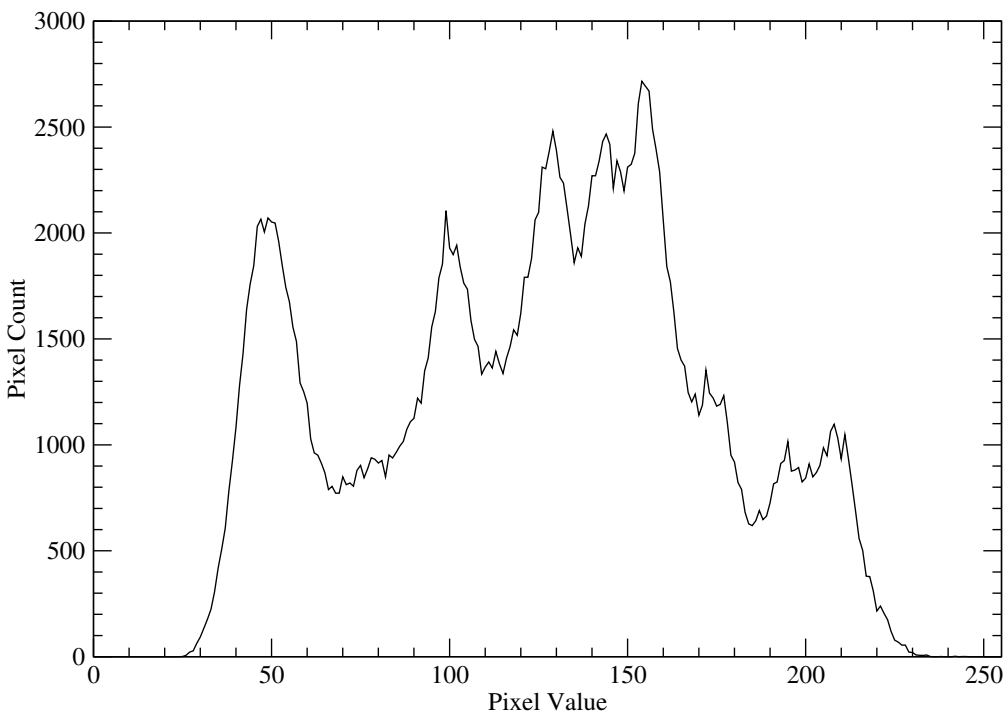

Histogram Of Transform Coefficients, Lena Image

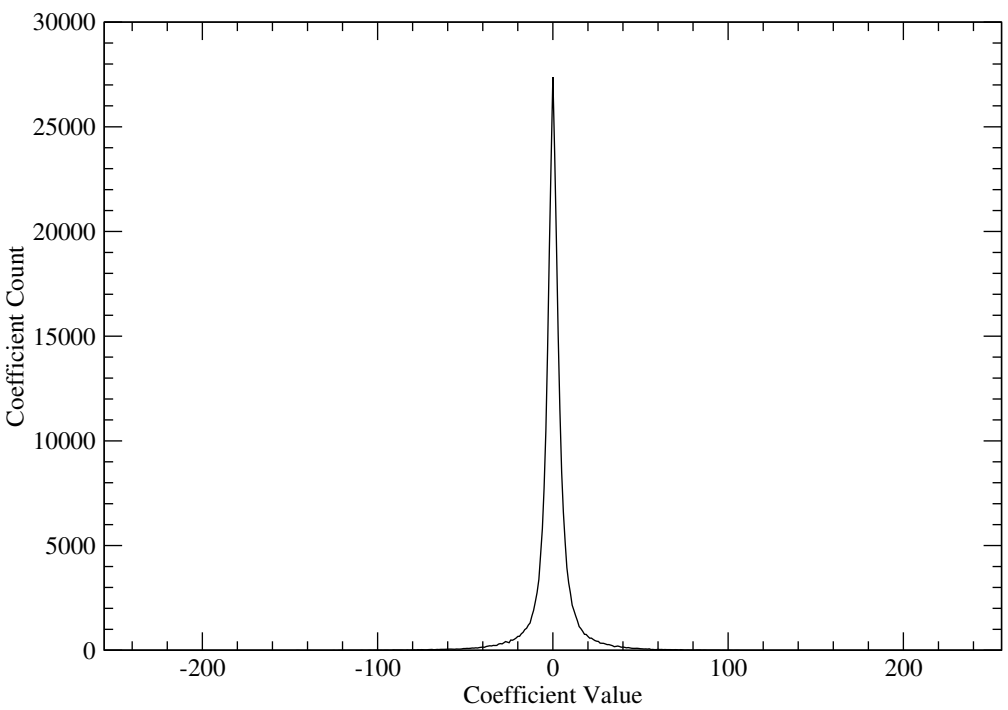

Figure 6.3: Histogram of Lena image (top), and histogram of transform coefficients after image decomposition by a wavelet transform. 
Figure 6.3 shows a histogram of a grayscale photograph before and after a wavelet transform. We see that before the transform the histogram is fairly wide and spread out. This is not good: the more different data values there are the worse the data will compress. After the transform we see that all of the coefficients are grouped around 0, with most of the coefficients being at or near zero. This is good: the high level of redundancy in the coefficients will allow them to be compressed more. An entropy measurement of the data before and after transformation will indicate that the transform coefficients will compress much better.

One of the most useful characteristics of a wavelet transform is the fact that it provides us with a representation of the data broken down into levels of increasing detail and decreasing significance. Again referring to figure 6.1, the lowest level subbands (i.e. the LL, LH, and HL regions produced earlier in the decomposition) contain data that is least important to the image, and the higher-level subbands contain information that is more important. A change at a higher level subband will propagate to more of the reconstructed image and therefore have a larger effect. A change at a lower-level subband will have less of an impact on the overall image.

This level-of-detail representation has some advantageous properties. The first thing to notice is that fully three-fourths of the wavelet coefficients are at the lowest (i.e. least-significant) level of detail. Lossy coding techniques can take advantage of this: the entropy of the coefficients can be reduced by quantization or thresholding without adversely affecting the quality of the reconstructed image. Quantization is the process of breaking up a continuous range into nonoverlapping subranges, such that all values $v_{i}$ that lie in the subrange map to a specific value $v^{\prime}$, also in the subrange. So for example we might specify that all coefficients $v_{i}$ that lie in the range $40 \leq\left|v_{i}\right| \leq 50$ be set to 42. A common, simple way for quantizing is to zero the lower $n$ bits of each coefficient. Thresholding is the process of zeroing each coefficient whose absolute value is less than or equal to some threshold $T$.

This level-of-detail representation also allows for the creation of data compression methods, such as Zerotree encoders [34, 26, 38, 46], that use wavelets to encode data 
such that the most important parts are encoded first, and the remainder of the data is encoded in order of decreasing importance. During decoding (or transmission) the process may be terminated at any point, and the reconstructed data is guaranteed to be the best possible given the amount of data transmitted.

A wavelet transform may also be used for automatic generation of mipmap images. A mipmap (see [56] for an explanation) is a representation of an image at increasingly coarse levels of detail. These images are typically precomputed, that is, the various images (one at each level of detail) are created in advance by progressive coarse filtering. Mipmaps are used in computer graphics as an antialiasing technique when rendering textures. When rendering a textured object that is "far away" a texture image from the mipmap's coarse level of detail is used. As the object comes closer to the viewer, increasingly finer images from the mipmap are used for texturing.

To use a wavelet transform for generating a mipmap, a detailed, base image can be decomposed, and the coefficients stored in memory, in compressed form if desired. Initially the coarse image is used, but if a more detailed image is needed the next subband may be decompressed and the inverse transform performed on one level.

\subsection{Dynamic Range Expansion}

One difficulty encountered when using wavelet transforms is Dynamic Range Expansion, or DRE (for a discussion of dynamic range expansion and its effects see [31]). Practically speaking, dynamic range expansion means that if the inputs to a wavelet transform are $n$ bits wide, the transform coefficients will require at least $n+1$ bits to represent. This extra bit presents some problems. If the transform is being performed in hardware (such as a video frame buffer) that has a channel width limited to $n$ bits, some data loss will result due to forced truncation of the coefficients. If custom hardware implementing the transform is to be designed and built, circuitry for handling the extra bit adds complexity and cost to the design. If the transform is implemented on a modern computer there is a potential for inefficient use of resources. Computers allocate storage in 8-bit bytes. If the inputs to a transform are 8 bits, the 9 -bit outputs will each require 16 bits of storage. 
This is potentially detrimental in an embedded computing environment.

What causes DRE? Taking the Haar transform as an example, if we assume that we have data values that require a minimum of $n$ bits each to store and if we plot the domain of all possible transform inputs $(A, B)$ we see from figure 6.4 that the domain is a square, centered at the origin, with an edge length of $2^{n}$. If we plot the range of all points $(L, H)$, given the domain, we see that the range is simply the domain, rotated 45-degrees about the origin. From figure 6.4 we see the cause of DRE: since the domain is a square, after rotation the corners lie outside the original domain area. The range, as measured along the axes, is larger than the domain. These values that lie towards the corners of the range require $n+1$ bits of storage.

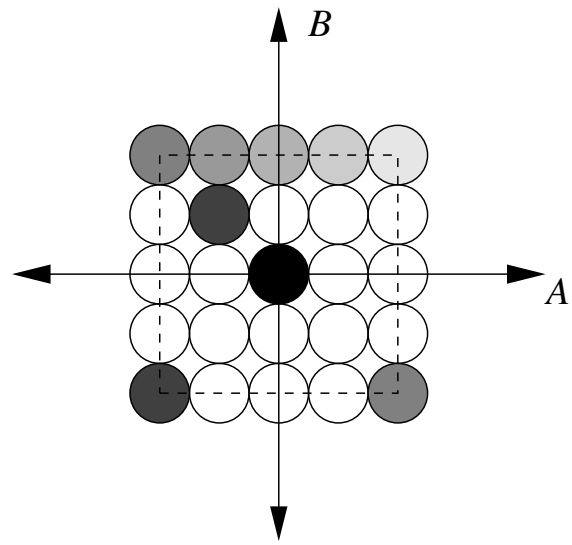

Haar Domain

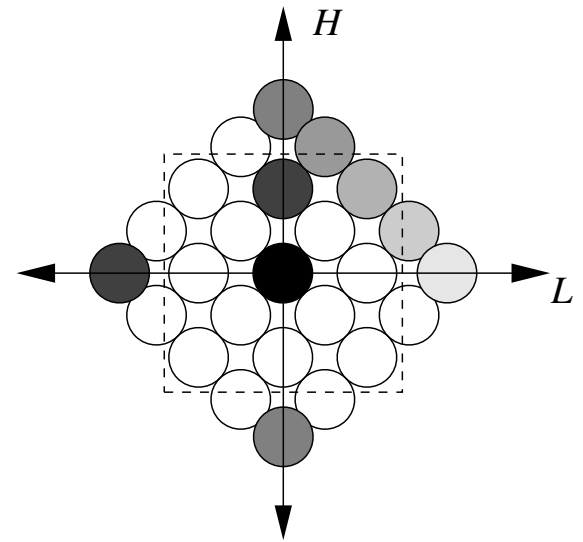

Haar Range

Figure 6.4: Dynamic Range Expansion in the Haar transform.

The Haar transform, although illustrative of what causes DRE, is not the best example because it always produces real-valued coefficients. Real-valued coefficients can be scaled, and so DRE is not really a problem here. However, DRE is a problem when one desires an integer-to-integer transform.

If the normalization by $\sqrt{2}$ is removed from equations 6.1 and 6.2 we have the non-normalized Haar transform of equations 6.3 and 6.4. The non-normalized Haar transform is a real-to-real or integer-to-integer transform. 


$$
\begin{aligned}
& L=A+B \\
& H=B-A
\end{aligned}
$$

If we plot the domain and range of the non-normalized Haar transform (see figure 6.5) we see that, like the Haar, it is a 45-degree rotation about the origin, but the distance between the points is expanded by a factor of $\sqrt{2}$, as expected. The range of the low- and high-pass values, as measured along the axes, is now twice that of the domain. In the case of the integer-to-integer non-normalized Haar transform it seems that we are stuck: DRE cannot be completely removed without producing real-valued coefficients.

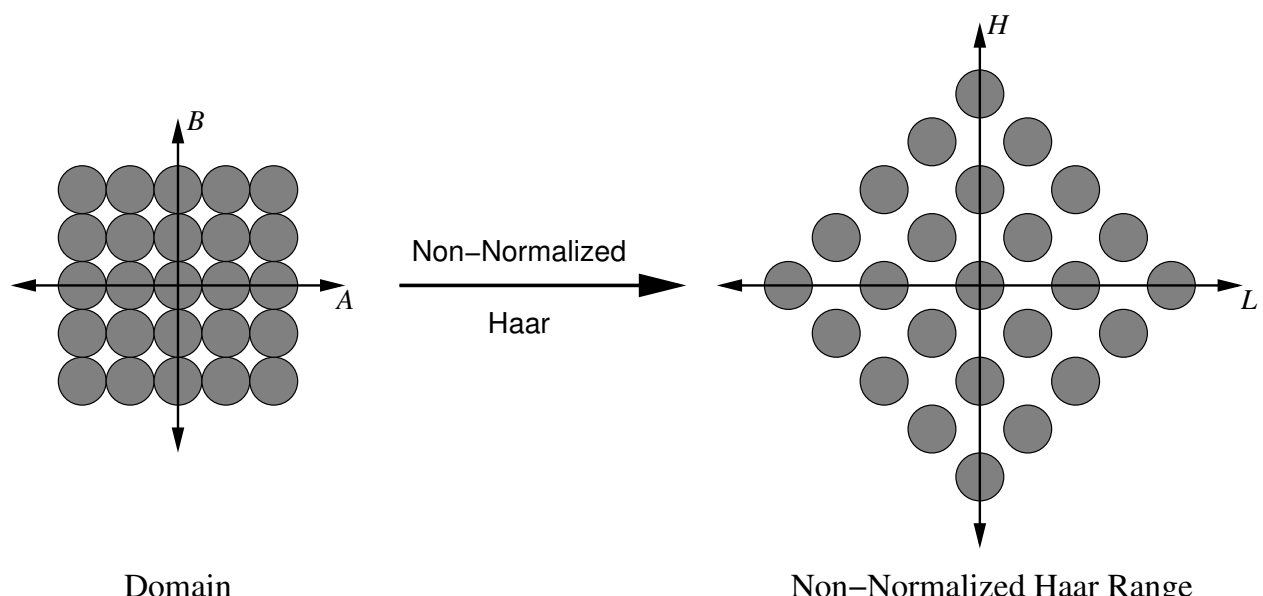

Figure 6.5: The non-normalized Haar transform.

\subsection{Removing DRE: The S-Transform}

Looking again at figure 6.5, we see that not all integer positions lying within the nonnormalized Haar range are occupied. In fact, the number of occupied positions in the range is equal to that of the domain. The range is just spread out over a larger area. The S-Transform [24] is an integer-to-integer transform closely related to the Haar that eliminates DRE in the low-pass coefficients by taking advantage of the extra space in the non-normalized Haar range. 


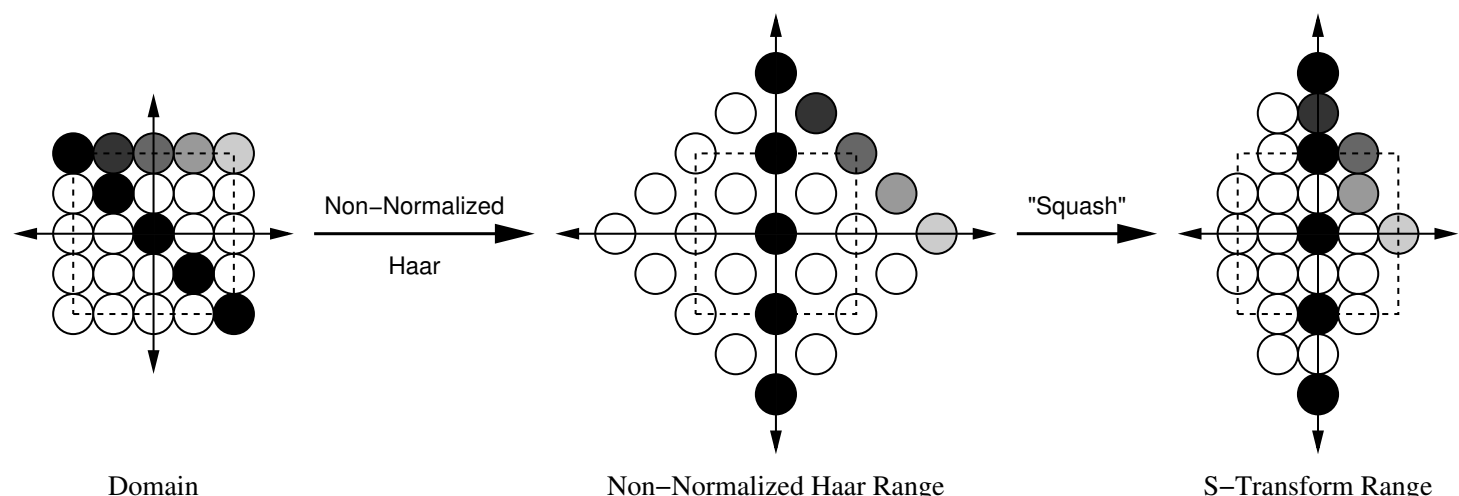

Figure 6.6: Eliminating low-pass dynamic range expansion by "squashing".

The S-transform is conceptually a two-step process, as illustrated in figure 6.6. First, each pair of inputs $(A, B)$ is transformed via the non-normalized Haar transform. Second, the range is "squashed" in the low-pass direction, eliminating the space between the points. This transform is accomplished via equations 6.5 and 6.6. Note that for any pair of inputs to the non-normalized Haar transform, $H$ and $L$ have the same leastsignificant bit (LSB). Because of this the low-pass values can be squashed without loss of information (the LSB needed to completely reconstruct the "unsquashed" $L$ can be taken from $H)$.

$$
\begin{aligned}
L & =\left\lfloor\frac{A+B}{2}\right\rfloor \\
H & =B-A
\end{aligned}
$$

The S-Transform has seen a lot of use because it is easy to implement and extremely fast. Unfortunately, the S-Transform produces high-pass coefficients with an expanded range. Eliminating DRE in the low-pass values is desirable because the lowpass range will be the same at each iteration of the transforms, and so at each stage of the wavelet decomposition the high-pass coefficients, although expanded, will all fall into the same range. However, the high-pass coefficients are of greater interest, as these are what remains when the transform terminates. It would be nice to eliminate DRE in both the low- and high-pass coefficients. 


\section{Chapter 7}

\section{$N$-bit to $N$-bit Transforms}

\subsection{More on Dynamic Range Expansion}

An $n$-bit to $n$-bit transform is one that has no dynamic range expansion. If the data values used as transform inputs are $n$-bits wide, the resulting coefficient outputs are also $n$-bits wide. Be careful not to confuse " $n$-bit to $n$-bit" with "integer-to-integer". As shown in section 6.5 a transform may be integer-to-integer and have dynamic range expansion; indeed, this is usually the case.

Dynamic range expansion (DRE) presents some hurdles when performing a transform. The first is that DRE may require a transformation to use twice as much memory as the data being transformed. For example, if an 8-bit grayscale photograph is transformed using the S-transform, the 9-bit coefficients will require 16 bits each to store, since modern

computers allocate storage in 8 -bit bytes. A raw image requiring $1024^{2}$ bytes of storage will generate coefficients requiring $2 \times 1024^{2}$ bytes.

It is also possible that the data being transformed must be sent over an $n$-bit channel. If the channel is $n$ bits wide, and the transformed data $n+1$ bits wide, we have to determine what to do with the extra bit. It can be truncated, but that results in automatic data loss. This may well be undesirable, especially in circumstances such as medical imaging. The transform coefficients can be bit-packed together, and the packed stream can be sent in 8 -bit pieces. This works, but is a slow process. It would be best if 
we didn't have that bit to worry about in the first place.

Recall from section 1.2 that our interest in $n$-bit to $n$-bit transforms is motivated by the need to transform an image in graphics hardware and send the coefficients over a DVI channel [23], which is 8 -bit. Thus for best performance we need an $n$-bit to $n$-bit transform.

\subsubsection{Can Dynamic Range Expansion be Eliminated?}

It would be nice if DRE could be completely eliminated. This would allow a transform to be performed more compactly and quickly, and simplify the process of working with a transform in hardware. Can DRE be removed?

The answer at first glance is yes. If we reexamine the diagrams of the Haar and non-normalized Haar transforms, we see that the number of points in the domain is equal to the number of points in the range; their arrangement in the range differs only by a rotation and possible expansion. Thus, if a new arrangement of the range points can be found such that (1) the arrangement fits into the exact same area as the domain and (2) the new arrangement results in the desired transform properties (e.g. data decorrelation) then we will have created a transform without DRE. As such, an $n$-bit to $n$-bit transform makes the range a permutation of the domain. How this permutation is done differentiates the different $n$-bit to $n$-bit transforms.

\subsubsection{Review: The Method of Chao, Fisher, and Hua}

To our knowledge, prior to our work in this area there was only one published method on an $n$-bit to $n$-bit transform. This was the method first released by Chao and Fisher in a preprint [12], and later published by Chao, Fisher, and Hua [13] (although we note that Swanson and Tewfik [51] presented a wavelet decomposition for binary bilevel images that has no dynamic range expansion). The preprint identifies this method as being suitable for lossy and lossless coding, the final published version states it is suitable for lossless coding

only (the final version is correct, as will be shown later). The method of Chao, Fisher, and Hua $(\mathrm{CFH})$ takes advantage of modular arithmetic to eliminate dynamic range expansion. 
Given a bit width $n$, the range of representable signed values is $\left[-2^{n-1}, 2^{n-1}-1\right]$. Given inputs $A$ and $B$ the $\mathrm{CFH}$ transform is computed as in equations 7.1 and 7.2 :

$$
\begin{aligned}
H & =(B-A) \bmod 2^{n} \\
L & =\left(\left\lfloor\frac{H}{2}\right\rfloor+A\right) \bmod 2^{n}
\end{aligned}
$$

where we use the standard convention that $-x \bmod 2^{n}=2^{n}-x$ for $0<x<2^{n}$.

The CFH method has an aliasing problem that causes undesirable behavior when the difference between $A$ and $B$ overflows and wraps around, causing $H$ to have a sign opposite the expected. $\mathrm{CFH}$ is unable to distinguish large positive numbers from small negative ones (and vice--versa) because they have the same binary representation. For example, if $n=8$, the range is $[-128,127]$. If $A=-1$ and $B=127$, the CFH method computes $H=-128$, which is nowhere near the difference between $A$ and $B$. The $L$ value is generally considered to be an "average" of $A$ and $B$, but because of $H$ being far from the expected value, $L$ is computed as -65 , which is also far from its expected value. If the wavelet coefficients are kept lossless then this aliasing does not cause any problems and the original image can be reconstructed. However, if a lossy method is used to encode the coefficients then severe artifacts may appear in the reconstruction. Many continuoustone images do not have adjacent pixel pairs with differences wide enough to cause this behavior, so in practice CFH works well for a wide range of images, reconstructed both with and without loss. However, this aliasing problem is a fundamental weakness in the method, and means that the CFH transform is not continuous.

The lookup tables for the CFH transform are given in figure 7.1. The origin of each table is its center, with the A and L-axes being horizontal. An examination of these lookup tables shows the aliasing problem: it creates discontinuities, as evidenced by the abrupt transitions. Examining the inverse transform table, should a coefficient pair $(L, H)$ fall into one of the four discontinuous regions, quantization or thresholding of $H$ may pull the point across the discontinuity. Then upon reconstruction the resulting $(A, B)$ pair will have a value much different than expected. This can result in artifacts that ruin a reconstruction. This is more clearly illustrated in figure 7.2, and an actual example is given 

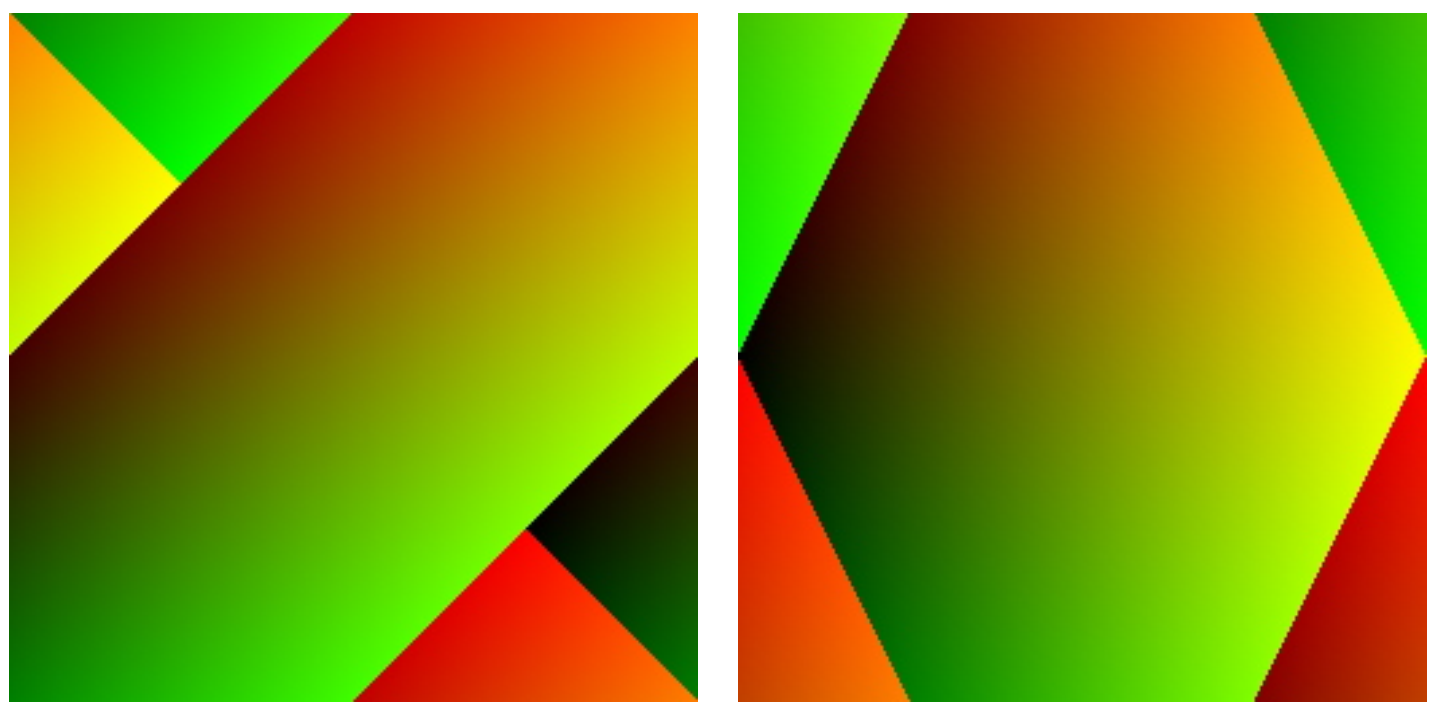

Figure 7.1: CFH transform lookup tables. The forward transform is on the left, and the inverse is on the right.

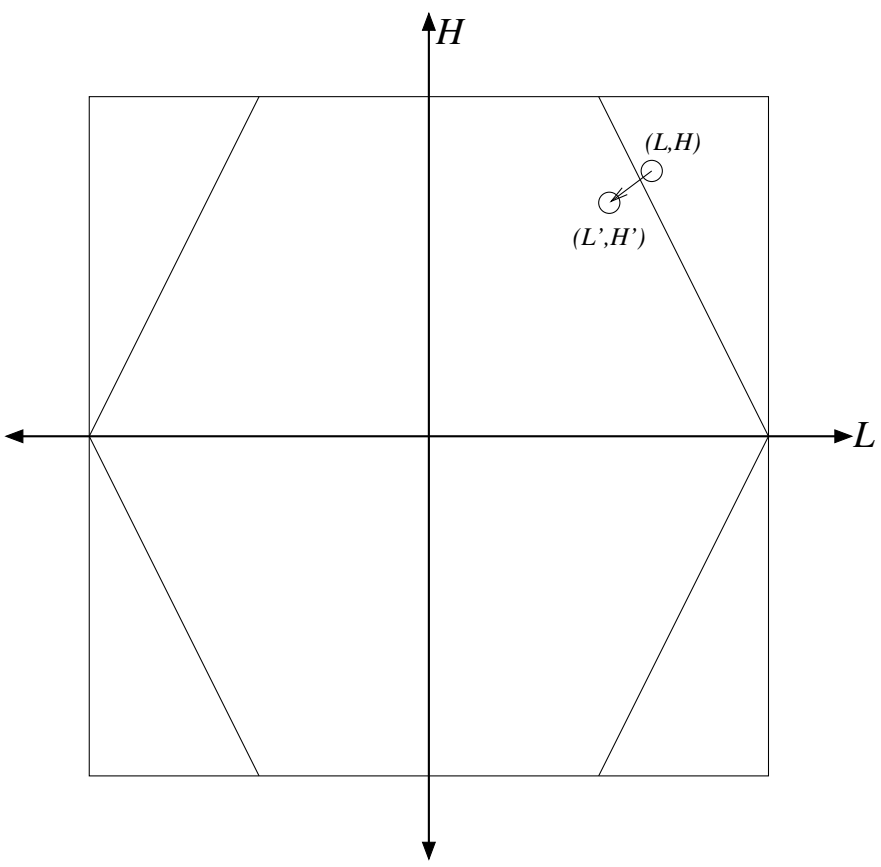

Figure 7.2: A diagram of the inverse $\mathrm{CFH}$ transform table, showing the axes and discontinuities. If a point $(L, H)$ is located near one of the discontinuities, a process such as quantization will pull $(L, H)$ across the discontinuity to a new point $\left(L^{\prime}, H^{\prime}\right)$. Artifacts will result upon reconstruction. 
in figure 7.3, where the Wedding photo has been thresholded to an equivalent entropy of one bit per pixel. The artifacts are obvious.

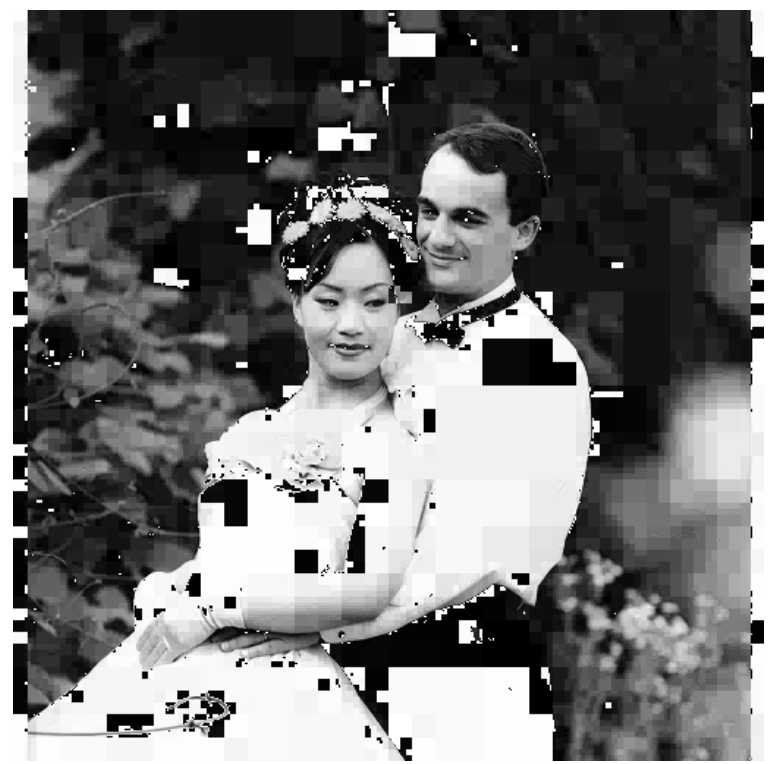

Figure 7.3: The Wedding photo, transformed by $\mathrm{CFH}$ and thresholded to an equivalent entropy of one bit per pixel.

\subsection{Transform Evaluation}

To evaluate a data transform's suitability it helps to have a set of metrics that can be used. These metrics, given data and a reconstruction of that data, produce a number indicating how close the reconstructed data is, or how faithful the reconstruction is, to the original. As with anything of this nature the weaknesses of these metrics need to be understood, and their results interpreted in light of those weaknesses and the uses to which the transforms will be put. In the data compression literature the metric typically used is the Peak Signal-to-Noise Ratio.

\subsubsection{Peak Signal-to-Noise Ratio}

A signal reconstruction is composed of original data (signal) and noise (errors, quantization artifacts, etc.). The Peak Signal-to-Noise Ratio (or PSNR), as its name implies, is a measure of the amount of signal present in a reconstruction vs the amount of noise present. 
The PSNR metric presumes that that a higher amount of signal indicates a better, or perhaps a more faithful, reconstruction.

$$
\begin{array}{r}
R M S E=\sqrt{\frac{1}{n} \sum_{i=1}^{n}\left(v_{i}-v_{i}^{\prime}\right)^{2}} \\
P S N R=20 \times \log \left(\frac{255}{R M S E}\right)
\end{array}
$$

Equation 7.3 is the equation for the root mean square error (RMSE) of the reconstructed image. In this equation $v_{i}$ indicates the original value of a pixel and $v_{i}^{\prime}$ is the reconstructed value. RMSE computes a value indicating how far the value of each reconstructed pixel deviates from the value of the original. Note that this definition of RMSE is almost identical to the definition of standard deviation used in statistics and error analysis.

From the RMSE we can define the PSNR as in equation 7.4. The value of the numerator of the fraction in this equation is set to be the largest possible value that can occur in the data being examined. In our case, since we use 8-bit grayscale images for testing, we set the numerator to 255 . In the worst case, $100 \%$ deviation in the reconstructed image, the RMSE will be computed as the maximum possible value 255 . In this case then the PSNR will be zero. In the best case of no deviation (i.e. the reconstructed image is identical to the original) the RMSE will be zero, and the PSNR will be infinite.

A higher PSNR indicates a reconstructed image whose average is closest to the average of the original image. It is generally accepted that a higher PSNR means a betterquality reconstruction. This is typically the case, but figure 7.4 gives one example of the dangers in relying on computed metrics for determining image quality. Reconstruction 1 has a PSNR of 12.01, and Reconstruction 2 a PSNR of 12.21. Although the PSNR indicates that in terms of preserving the original image the two reconstructions are essentially the same (with Reconstruction 2 being slightly favored), it should be obvious that for an observer Reconstruction 2 is a much better reconstruction.

The weakness of the PSNR metric is that it favors reconstructions that are closest to the average of the original image. A reconstruction that preserves few details but lies 


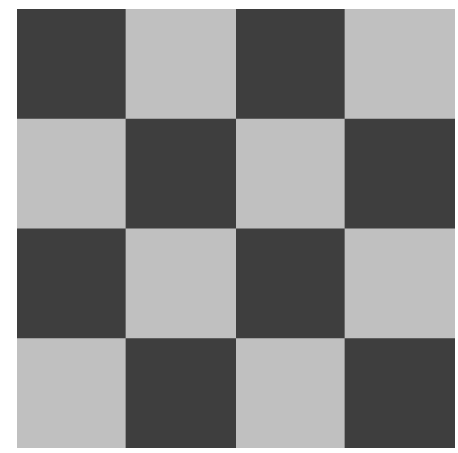

Original Image

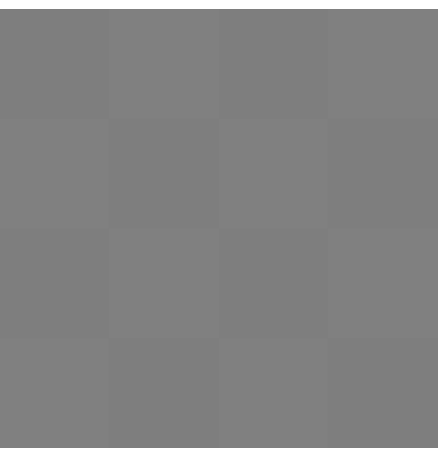

Reconstruction 1, 12.01

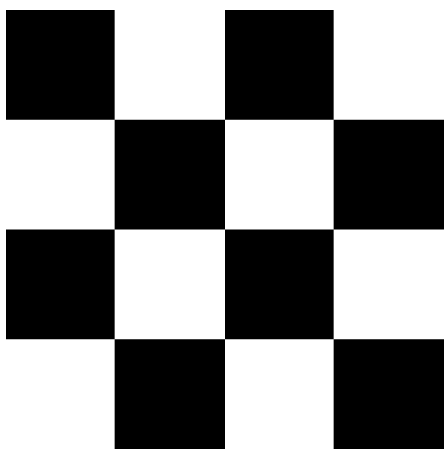

Reconstruction 2, 12.21

Figure 7.4: One of the dangers of relying on computed metrics to determine image quality. The PSNRs of both reconstructions are almost identical, but Reconstruction 2 is obviously a much better reconstruction.

close to the average of the original data will have a PSNR that is comparable to, or better than, a reconstruction that preserves details but lies far from the average.

\subsection{2 $L_{\infty}$ Error}

The $L_{\infty}$ error is defined by equation 7.5, and is a measure of the maximum distance that any reconstructed pixel value is from its original value. This measure is useful for determining how effective a transform is at keeping actual pixels close to their original value. With a metric like PSNR it is possible to have a reasonably good value when the reconstructed image has some artifacts or errors present. With the $L_{\infty}$ metric any artifact that severely alters the value of a pixel will result in a high error value overall. The obvious weakness in this metric is that if a reconstruction contains a single large error in one pixel - even if the rest of the image contains little or no error - the $L_{\infty}$ error value is high.

$$
L_{\infty}=\forall i: \max \left(\left|p_{i}-p_{i}^{\prime}\right|\right)
$$

\subsubsection{Images}

Although data transforms are applicable to a wide variety of data types we use images to illustrate our transforms and study their effectiveness, as with images the results are 
more obvious to most observers. This requires a collection of images on which to test. The sources for the images are listed in the Acknowledgements section at the beginning of this work. A description of the image categories is given here, and some examples of the categories are in figure 7.5. BW Lines: Some small images containing black and white lines. LineArt: Line art images. These have hard edges, lines, regions of solid color, gradient fills, etc. ObjectBank: Some simple computer-generated images. MRI: MRI images of a person's upper spine/neck. These are padded to $256^{2}$ resolution. ccitt: Some standard FAX test images. DB1_B: Fingerprint images. DB2_B: Same images as DB1_B, but enhanced and with altered dimensions. Photos: Photos, including standard tests images (e.g. Lena, Mandrill), ISO images (Woman, Bike), and some personal photographs (e.g. Wedding). r2_slices: Some random image slices from the LLNL PPM dataset. 

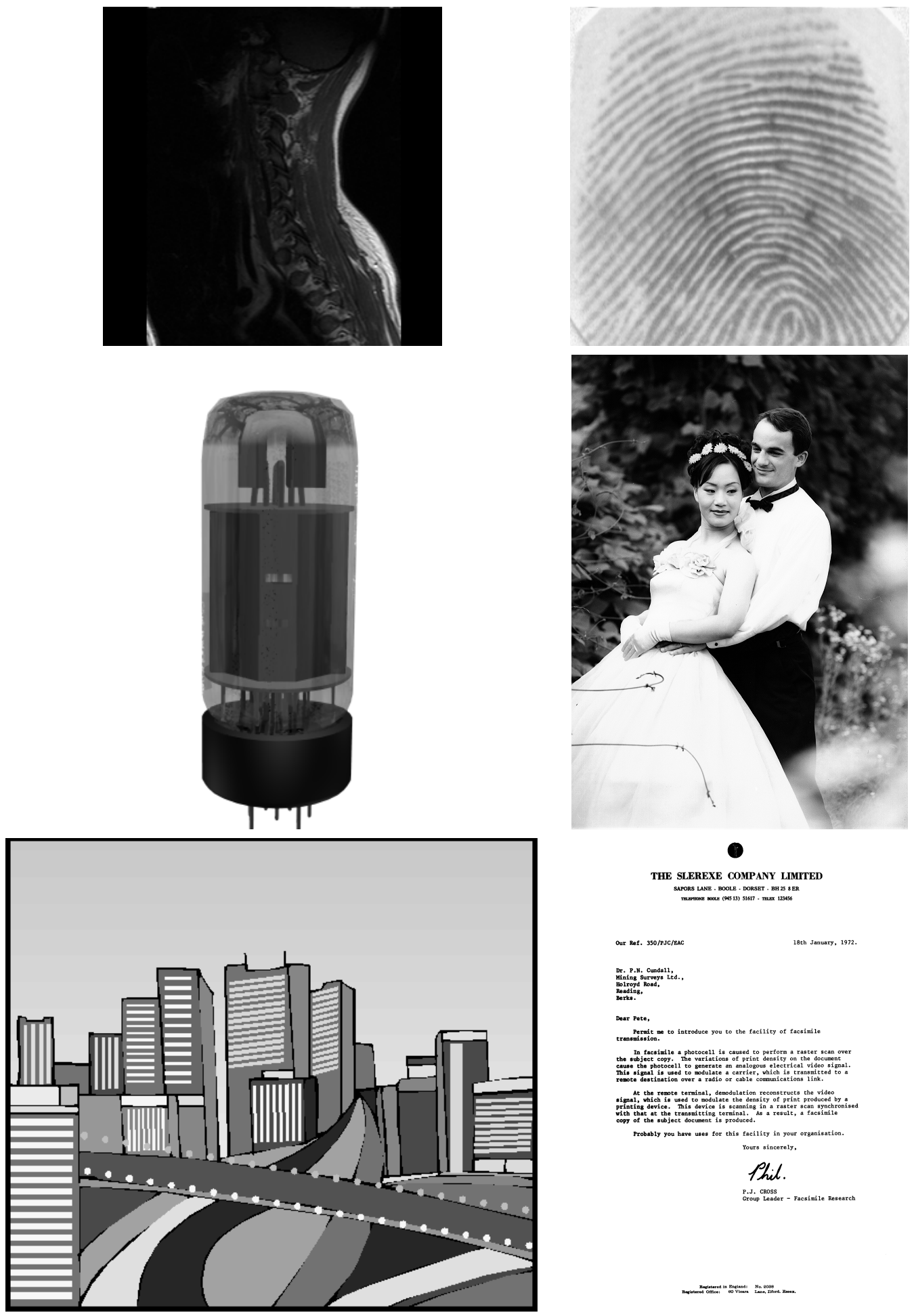

THE SLEREXE COMPANY LIMITED

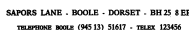

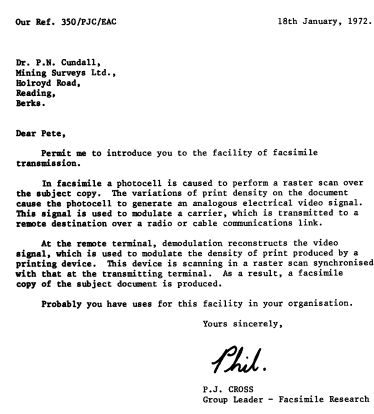

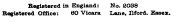

Figure 7.5: Test images. From top, left to right: MRI, Fingerprint, ObjectBank, Photos, LineArt, CCITT. 


\section{Chapter 8}

\section{Table-Lookup Haar}

Our first contribution to the field of $n$-bit to $n$-bit transforms is the Table-Lookup Haar method (TLHaar) [41, 42]. TLHaar is a transform that uses table lookups to perform its transform. It is extremely fast and produces coefficients that compress well. However, the TLHaar transform is very discontinuous and is in many ways inferior to the CFH transform (TLHaar was developed before we were aware of the CFH method). Nevertheless, the development of TLHaar was a useful and beneficial process, during which much was learned.

We saw in section 7.1.1 that an $n$-bit to $n$-bit transform has a range that is simply a permutation of the domain. TLHaar [41] is an integer-to-integer transform that, given a bit width $n$, uses a pair of procedurally-created square two-dimensional lookup tables (each with an edge dimension of $2^{n}$ ). One table, AB2LH, is a mapping $(A, B) \rightarrow(L, H)$ for the forward transform, and the table for the inverse, LH2AB, is a mapping $(L, H) \rightarrow(A, B)$. Each table is initialized with an identity transform, and then the rows and columns of the inverse transform table are sorted so that for any given two pairs of inputs their high- and low-pass values will have the same magnitude relationships as those in Haar. When a swap occurs in the inverse transform table, the corresponding entries in the forward transform table are also swapped. Because the tables are initialized with an identity transform no table entry will have a value outside the transform domain. Thus dynamic range expansion cannot occur. 


\subsection{Transform LUTs}

In order for the transform to be reversible there must be a 1:1 mapping between the domain and range. We therefore initialize each table with an identity transform prior to sorting, as this is the simplest 1:1 mapping. Given two pairs of inputs $\left(A_{i}, B_{i}\right),\left(A_{j}, B_{j}\right)$, the sort of the inverse transform table obtains the following two properties:

$$
\begin{gathered}
\forall \tilde{L}:\left|H_{i}\right| \leq\left|H_{j}\right| \Longleftrightarrow \tilde{H}_{i} \leq \tilde{H}_{j} \\
\forall \tilde{H}: L_{i} \leq L_{j} \Longleftrightarrow \tilde{L}_{i} \leq \tilde{L}_{j}
\end{gathered}
$$

where $H$ indicates a Haar high-pass coefficient, and $\tilde{H}$ indicates a TLHaar coefficient. The pseudocode for this procedure is the following:

do \{

For each L Column in LH2AB

Sort based on $|(B-A)|$

For each H Row in LH2AB

Sort based on $(A+B)$

\} while (there was a swap)

During the sort process whenever a swap occurs in HL2AB the corresponding entries in AB2HL are also swapped.

Figure 8.1 shows the lookup tables for the case when $n=8$. We see that the desired properties are obtained. The closer $A$ and $B$ are in value the closer $L$ is to their average, as evidenced by the smooth transition from 0 to 255 along the diagonal of the $A B \rightarrow L$ table. Likewise, the closer $A$ and $B$ are in value the closer $H$ is to zero, as evidenced by the black band along the diagonal of $A B \rightarrow H$.

Figure 8.2 shows the LH2AB LUT for $n=3$, with a column and row breakout for a particular table entry. Note how all column entries satisfy property 8.1 and all row entries satisfy property 8.2. Also, from figure 8.3 we see that this transform results in data decorrelation and is suitable for use in a data compression system. 

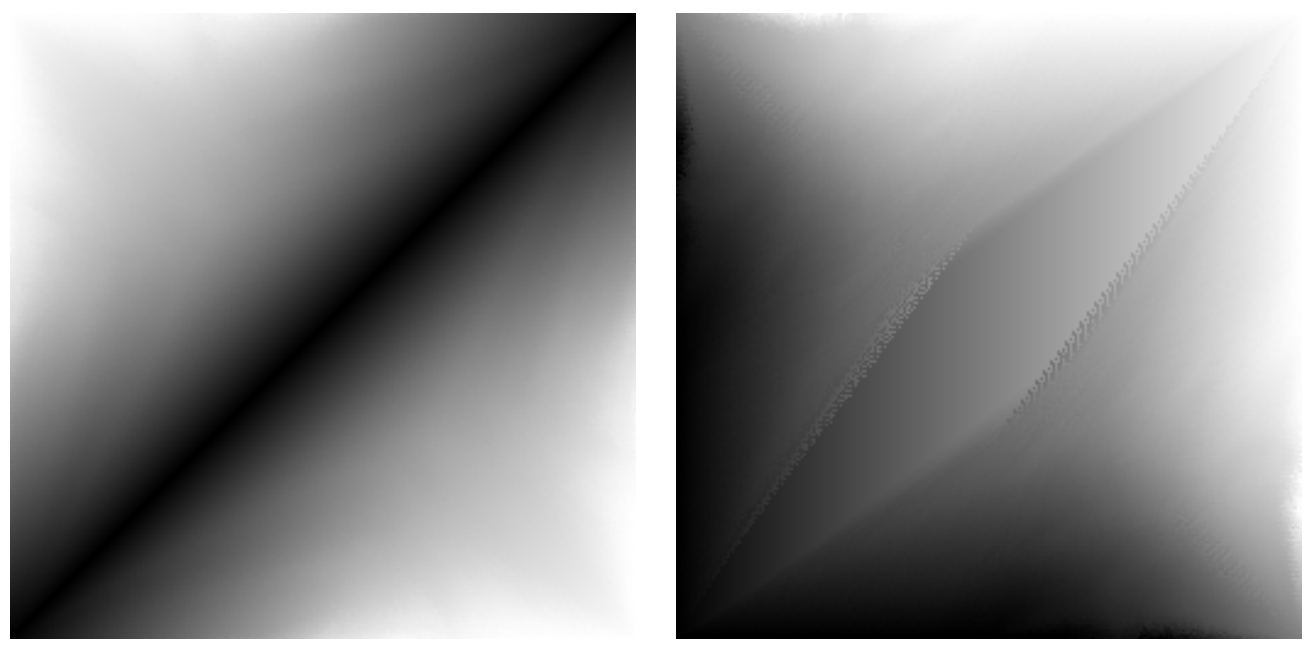

$$
A B \rightarrow H
$$

$$
A B \rightarrow L
$$

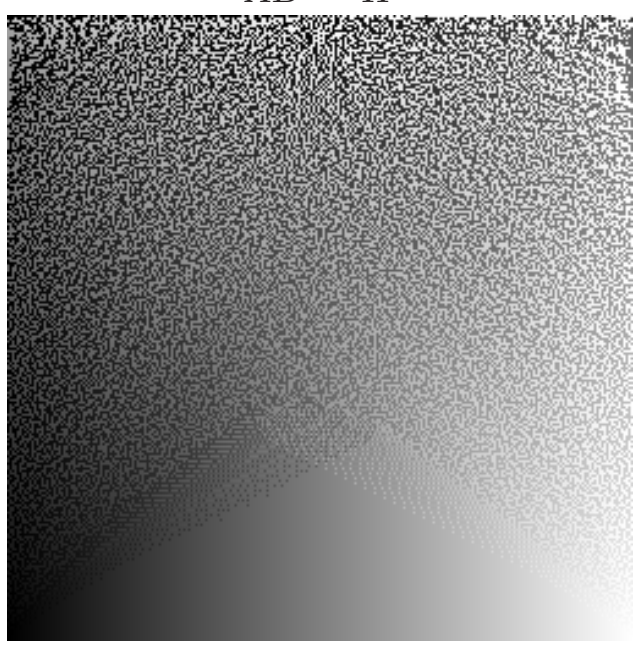

$$
L H \rightarrow A
$$

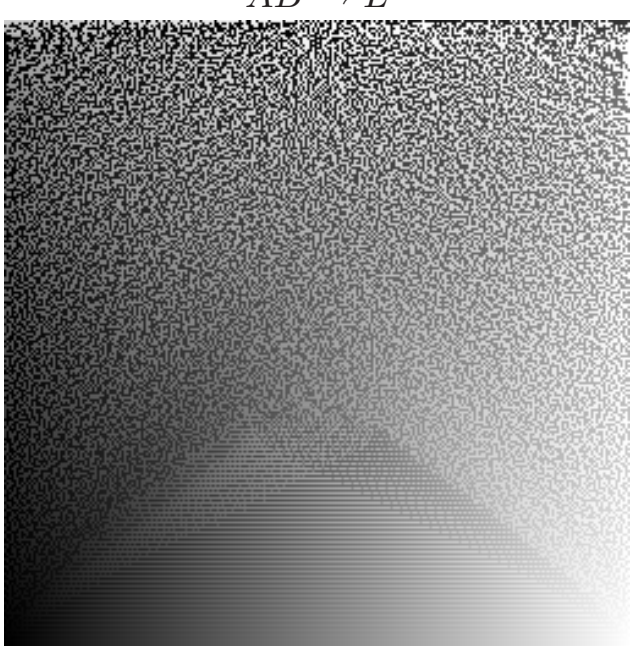

$$
L H \rightarrow B
$$

Figure 8.1: Forward and inverse transforms resulting from the sort. $A$ and $L$ are on the horizontal axes, and $B$ and $H$ on the vertical. The origin $(0,0)$ is the lower-left corner of each table. Black indicates a value of 0 , and white a value of 255 . 


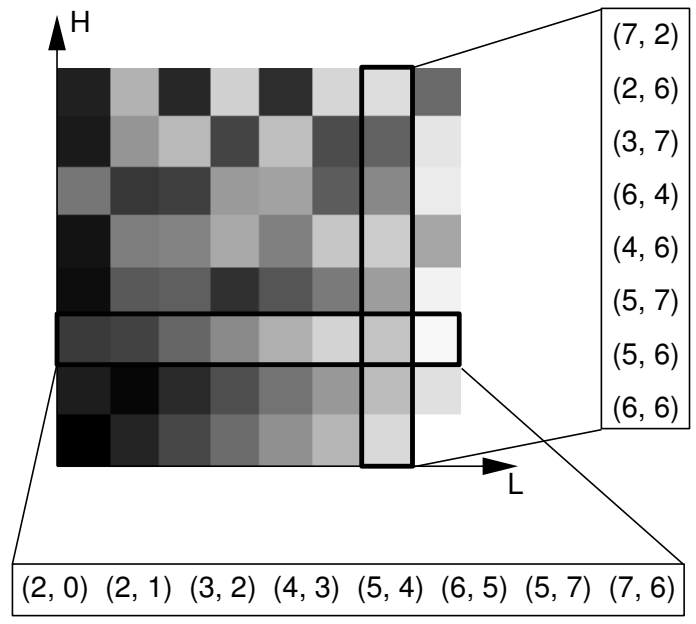

Figure 8.2: LH2AB LUT for $n=3$, showing a column and row breakout. Entries are $(A, B)$.

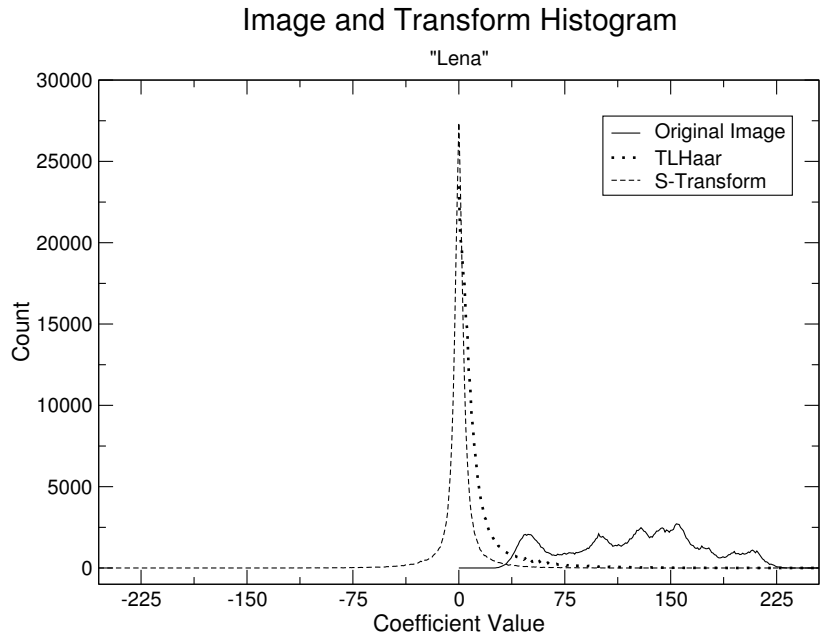

Figure 8.3: Histogram of the "Lena" image, before and after transformation. 

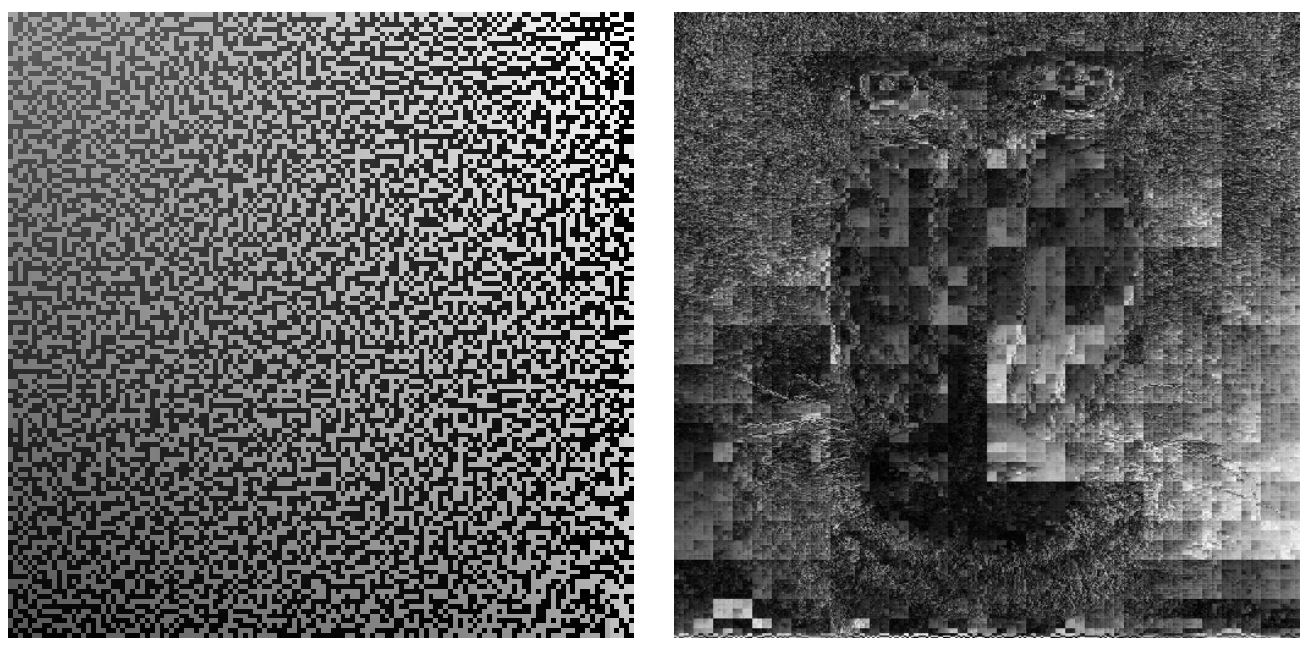

Figure 8.4: TLHaar's discontinuities, and their effects. The Mandrill reconstruction is after quantization to 6 bits.

It was not clear beforehand that this sort would converge. At this time we do not have a general proof that the sort will always converge, however we tested this process of creating tables for values $2 \leq n \leq 12$, and our tests indicate that in all cases the sort converges. We are unable to test further since when $n>12$ the tables become so large they are impractical. For $n=13$ a single LUT will contain over 67 million entries and be 256 megabytes in size.

\subsubsection{Discontinuities}

TLHaar is a discontinuous transform, and is therefore only suited for lossless coding. The left of figure 8.4 shows a close-up of the $L H \rightarrow A$ inverse transform table. The discontinuities are obvious and plentiful, and indicate that even a small quantization can result in a reconstructed value very different from the original. This results in severe artifacts, that usually ruin the image. For an example, the right side of figure 8.4 shows the Mandrill image reconstructed after quantization to 6 bits. 


\subsection{Sorted LUTs Are not Unique}

In section 8.1 we demonstrated the method used for creating the TLHaar LUTs, using a sort on an identity transform. If the LUTs are initialized with a 1:1 mapping different from our original method, will the sort still result in the same transform LUTs? The answer to this question is no, implying that LUTs satisfying relationships 8.1 and 8.2 are not unique. As a test we permuted one of the initialized LUTs before sorting, by randomly swapping entries. The corresponding entries in the other LUT were also swapped, maintaining a 1:1 mapping. We then performed our sort on the permuted tables. The resulting transform tables were different from those of our original method. By varying the number of swaps and the seed to the pseudorandom number generator we produced tables that were similar to each other, but not identical.

\subsection{TLHaar Implementation Optimizations}

Because TLHaar operates on and produces $n$-bit data, when $n$ is both a power of 2 and an integer size common in modern computer architectures (8-bit byte, 16-bit short integer, etc.) it is possible to store the low-pass and high-pass values in arrays of that integer type. This allows us to implement and take better advantage of some special optimizations. Here we describe optimizations made for an implementation that operates on 8-bit images.

We first altered how we perform table lookups in a row transform. Since input values $A$ and $B$ are adjacent in memory, instead of reading $A$ and $B$ separately and indexing the $\mathrm{AB} 2 \mathrm{HL}$ LUT with both (i.e. $H L=A B 2 H L[A][B]$ ) we cast the input array of bytes into an array of 16-bit short integers, and read $A$ and $B$ together as a single short $A B$. This allows us to perform a complete table lookup using fewer operations: $H L=A B 2 H L[A B]$.

We would like to use the above optimization when performing a transform in the column direction. The standard row transform operates on an image one row at a time, writing out the resulting low-pass values such that they are contiguous in the row direction. Thus a given image column is not contiguous in memory. To solve this when 

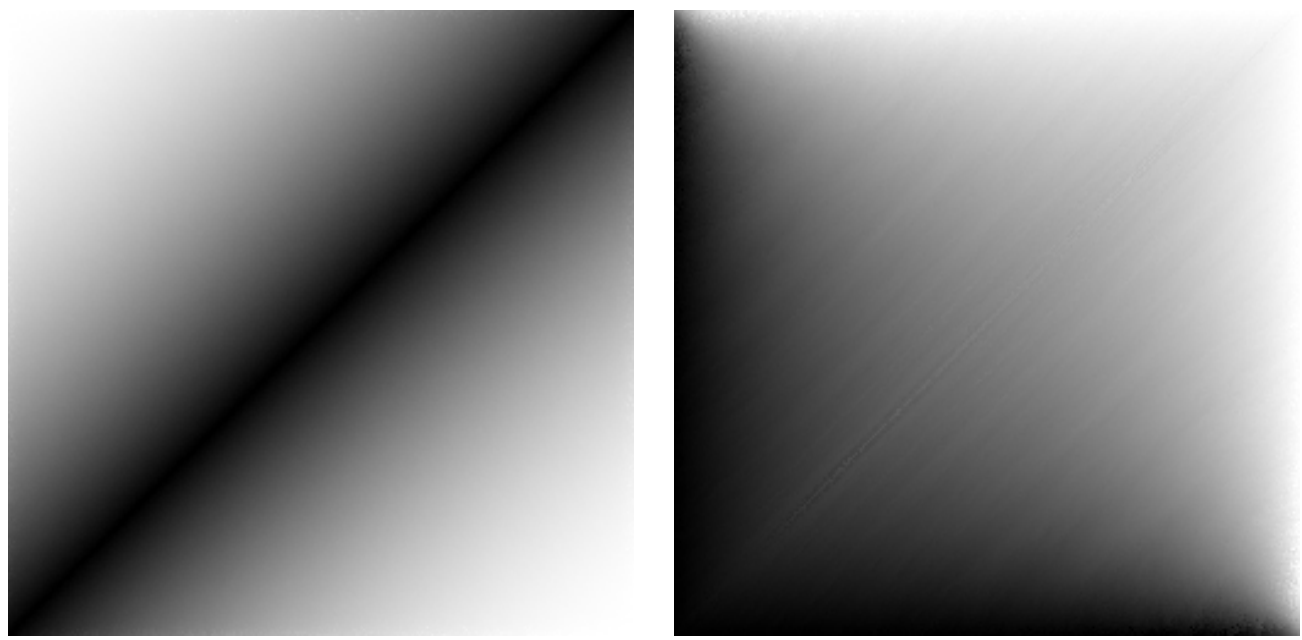

$$
A B \rightarrow H
$$

$A B \rightarrow L$

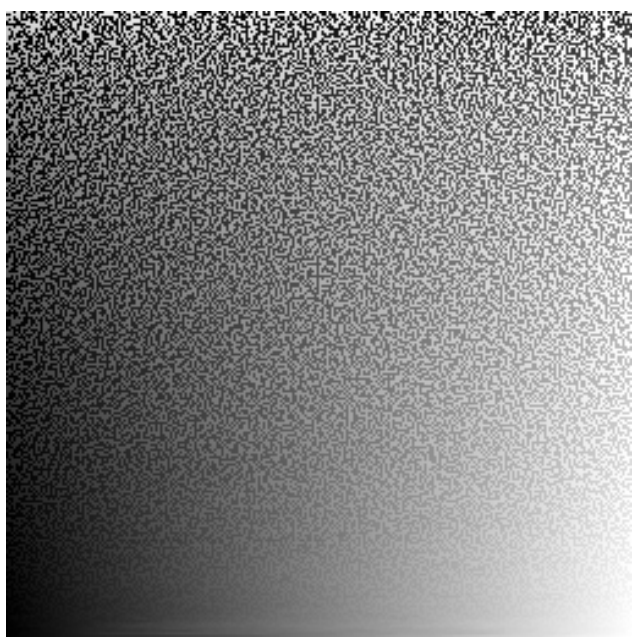

$L H \rightarrow A$

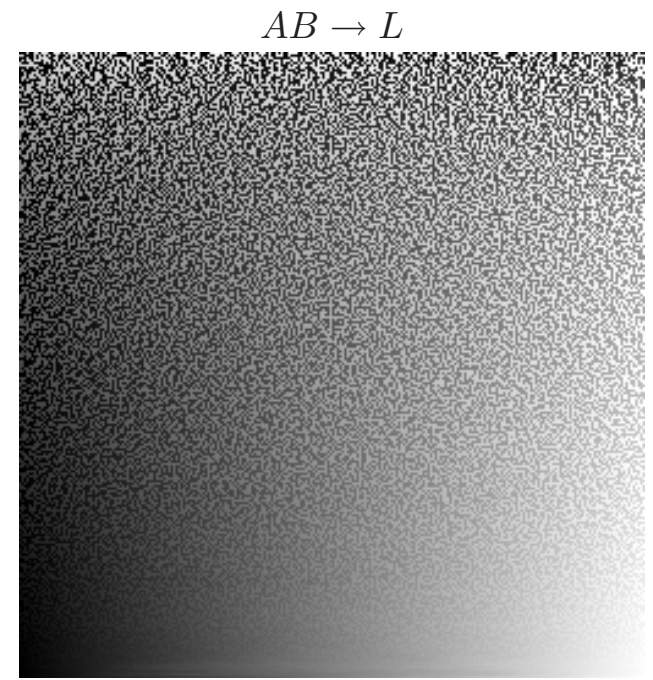

$$
L H \rightarrow B
$$

Figure 8.5: Transform tables resulting from a randomized initialization. 
performing a row transform we transform two rows at a time. Given the $k$-th pair of pixels from rows $i$ and $i+1$ we transform $A_{i} B_{i}$ and $A_{i+1} B_{i+1}$, and place $L_{i}$ and $L_{i+1}$ adjacent to each other in preparation for the column transform. The column transform can then proceed down columns in image space, but along adjacent memory locations. The idea behind the optimization is shown in figure 8.6. The right side shows the low-pass values ordered in memory.

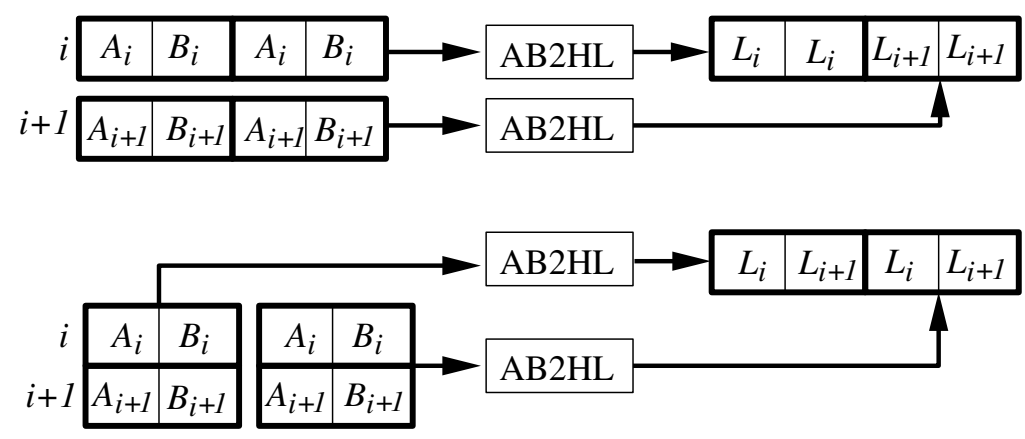

Figure 8.6: TLHaar row transform data reordering optimization. The top shows the normal transform procedure, and the bottom the reordering procedure.

\subsection{Results}

\subsubsection{Execution Time}

To compare execution times we implemented procedures to perform the S-transform and TLHaar transforms on 8-bit grayscale images, and optimized each separately. We also implemented versions of TLHaar and the S-transform that perform data reordering and operate on square images with edge lengths that are a power of 2. Timings were taken on a $550 \mathrm{MHz}$ PowerBook G4 running MacOS 10.1.5. The time given is an average over 10 transform runs, where a run transforms all images in a particular category, and includes only the time taken to transform the image. Execution times are given in table 8.1.

\subsubsection{Compressibility of Coefficients}

As our main interest in developing TLHaar was to create a fixed-width transform we are not greatly concerned about how efficiently TLHaar's coefficients compress compared to 


\begin{tabular}{|c||c|r|r|}
\hline \multicolumn{1}{|c||}{} & \multicolumn{2}{c|}{ Transform Time (sec) } & \\
\hline Category & TLHaar & S-transform & \% Gain \\
\hline \hline BW_Lines & 0.06368 & 0.06540 & 2.63 \\
LineArt & 0.15289 & 0.27621 & 44.65 \\
ObjectBank & 0.90947 & 1.08220 & 15.96 \\
MRI & 0.45442 & 0.55188 & 17.66 \\
MRI (reord) & 0.38121 & 0.51650 & 26.19 \\
ccitt & 1.31669 & 1.96779 & 33.09 \\
DB1_B & 0.25860 & 0.36670 & 29.48 \\
DB2_B & 0.31501 & 0.35577 & 11.46 \\
Photos & 1.02017 & 1.34319 & 24.05 \\
r2_slices & 0.31479 & 0.39773 & 20.85 \\
Power2 & 0.23322 & 0.31506 & 25.98 \\
Power2 (reord) & 0.20557 & 0.29665 & 30.70 \\
\hline
\end{tabular}

Table 8.1: Test image categories and transformation times. (reord) indicates execution time using the reordering method of section 8.3.

the S-transform. If the resulting compression ratio of TLHaar coefficients is within a percent or two of the rate obtained by the $\mathrm{S}$-transform, we are content. To get a feel for how coefficients generated by TLHaar compress compared to those produced by the S-transform we transformed the test images and then compressed the results using three freely available compression programs: gzip ${ }^{1}$, bzip ${ }^{2}$, and an arithmetic coder available from Alistair Moffat ${ }^{3}$. We used binary and byte arithmetic encoding.

To gauge the effect of sign bits on the compressibility of S-transform coefficients we compressed them in two ways. In the first method the coefficient magnitudes were written as a stream of bytes and compressed, and the sign bit for each nonzero magnitude was appended uncompressed. In the second method coefficient magnitudes were written as a stream of bytes, and then a binary stream consisting of the sign bits of all nonzero magnitudes was appended. The combined stream was then compressed.

Due to lack of space we present in table 8.2 results only for the former method, as it presents the S-transform more favorably and the comparison to TLHaar is more fair. Generally when the latter method is used, the S-transform coefficients do not compress nearly as much as the TLHaar coefficients. With byte arithmetic coding the TLHaar

\footnotetext{
${ }^{1}$ http://www.gzip.org

${ }^{2}$ http://sources.redhat.com/bzip2/

${ }^{3}$ http://www.cs.mu.oz.au/ alistair/arith_coder/
} 
coefficients always compress to a smaller size. Some example results in this case are TLHaar being $4.45 \%$ better in the Photos category and $7.23 \%$ better in the MRI category.

\begin{tabular}{|c|c|c|c|c|c|c|}
\hline Image & TLHaar & S-transform & $\%$ Gain & TLHaar & S-transform & $\%$ Gain \\
\hline & \multicolumn{3}{|c|}{ gzip } & \multicolumn{3}{|c|}{ bzip } \\
\hline BW_Lines & 360972 & 466731 & 22.66 & 391737 & 500173 & 21.68 \\
\hline LineArt & 419235 & 528964 & 20.74 & 445387 & 515182 & 13.55 \\
\hline ObjectBank & 3131056 & 3341803 & 6.31 & 3115625 & 3258474 & 4.38 \\
\hline MRI & 5477859 & 5381934 & -1.78 & 5281869 & 5190000 & -1.77 \\
\hline ccitt & 1157989 & 1557889 & 25.67 & 1013614 & 1454085 & 30.29 \\
\hline DB1_B & 5016509 & 5086675 & 1.38 & 4973728 & 4853424 & -2.48 \\
\hline DB2_B & 5820744 & 6165921 & 5.60 & 6175512 & 6353409 & 2.80 \\
\hline Photos & 15029496 & 14912472 & -0.78 & 14963474 & 14651157 & -2.13 \\
\hline \multirow[t]{2}{*}{ r2_slices } & 777975 & 801756 & 2.97 & 785564 & 783836 & -0.22 \\
\hline & \multicolumn{3}{|c|}{ Binary Arithmetic } & \multicolumn{3}{|c|}{ Byte Arithmetic } \\
\hline BW_Lines & 391472 & 494289 & 20.80 & 377422 & 496202 & 23.94 \\
\hline LineArt & 509478 & 627262 & 18.78 & 535573 & 677915 & 21.00 \\
\hline ObjectBank & 3290576 & 3474223 & 5.29 & 3620160 & 3771556 & 4.01 \\
\hline MRI & 5691930 & 5627898 & -1.14 & 5408487 & 5407783 & -0.013 \\
\hline ccitt & 1199500 & 1652339 & 27.41 & 1288297 & 1969556 & 34.59 \\
\hline DB1_B & 5673653 & 5346789 & -6.11 & 4796648 & 4703212 & -1.99 \\
\hline DB2_B & 6415074 & 6803730 & 5.71 & 5679509 & 5946472 & 4.49 \\
\hline Photos & 15234370 & 14873308 & -2.43 & 14285797 & 14090810 & -1.38 \\
\hline r2_slices & 828160 & 850696 & 2.65 & 823509 & 834297 & 1.29 \\
\hline
\end{tabular}

Table 8.2: Compressed category sizes (in bytes).

Results for TLHaar in table 8.2 are when using unpermuted tables, as described in section 8.1. Currently it seems that while randomly permuted and sorted tables may give better performance for continuous-tone images (photographs), they are horrible when it comes to things that have a lot of contrast, lines, hard edges, etc. In general, starting with an identity transform and then sorting seems to offer the best all-around performance.

We do not include the size of the transform LUTs in the TLHaar coefficient sizes. The tables are a static part of the transform process and are therefore known ahead of time, so in a coding application the tables do not need to be sent as part of the encoded data. 


\subsection{Conclusions and Possible Extensions}

The development of TLHaar was very fruitful, in that it led to the development of the PLHaar transform, which will be reviewed next. However, TLHaar's shortcomings may mean that it will not be suitable for actual use. In particular, TLHaar must be used with a lossless coding system, and if lossless coding is required then the $\mathrm{CFH}$ transform is probably more suitable. Both $\mathrm{CFH}$ and TLHaar require the same amount of space for lookup tables, but $\mathrm{CFH}$ can be performed by direct computation as well, so it is more versatile. Also, its performance with continuous-tone images is likely to be at least as

good as, if not better than, TLHaar (see figure 9.5 for example). Finally, when using a lossless coding method, should data corruption occur $\mathrm{CFH}$ is more tolerant of errors than TLHaar.

There is one avenue of future research that may prove worthwhile. Up to this point, we have created TLHaar tables using the sort method described in section 8.1, in order to mimic the Haar transform. Tests have shown that a given set of data only touches part of the tables during the transform process (see figure 8.7). So we have the question: can we move away from a procedurally created LUT and instead create a table that is custom-optimized (in terms of minimizing entropy) for a specific set of images? The process of investigating this question will likely involve developing a method using both simulated annealing and genetic algorithms and be computationally intensive, requiring parallel computation to solve more quickly. It is very possible that it will be impractical (or impossible) to create tables that are suitable for a large number of images, and some preliminary work indicates that this may be the case. 


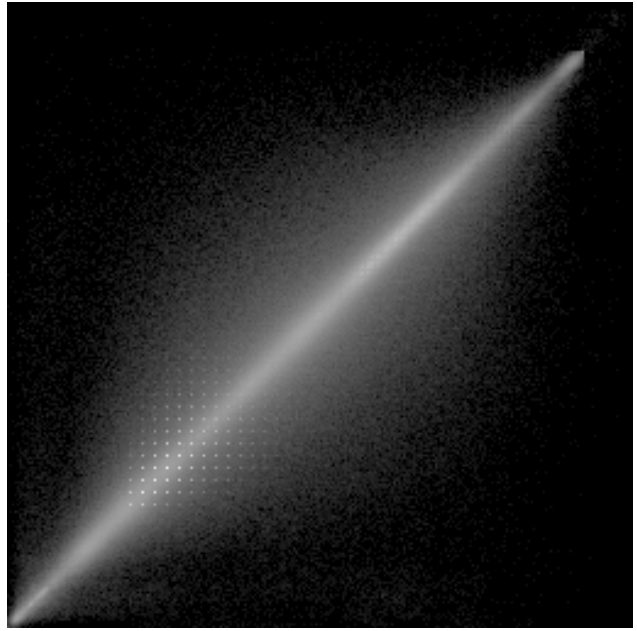

Photos

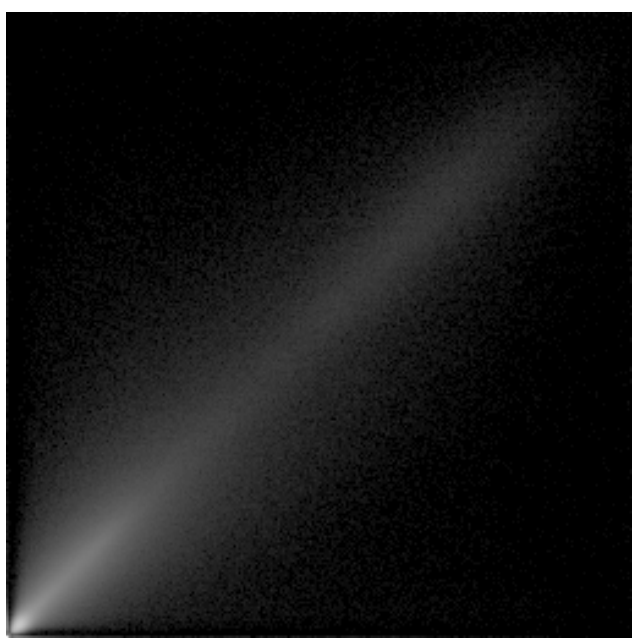

MRI

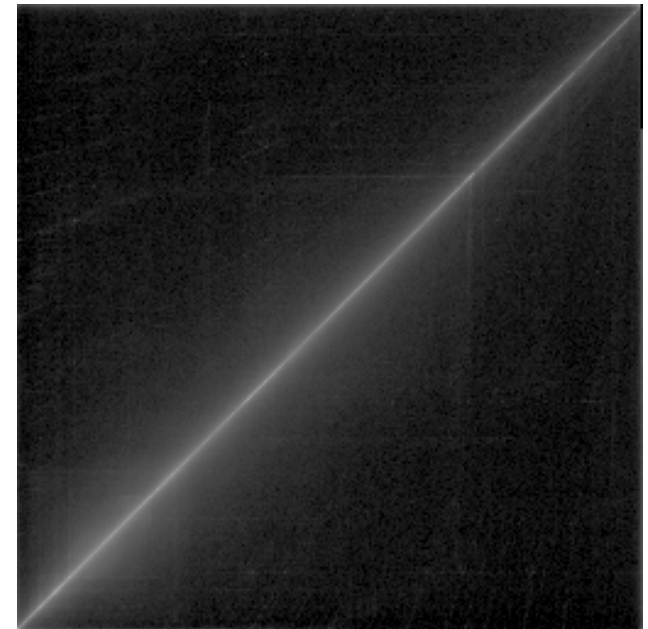

ObjectBank

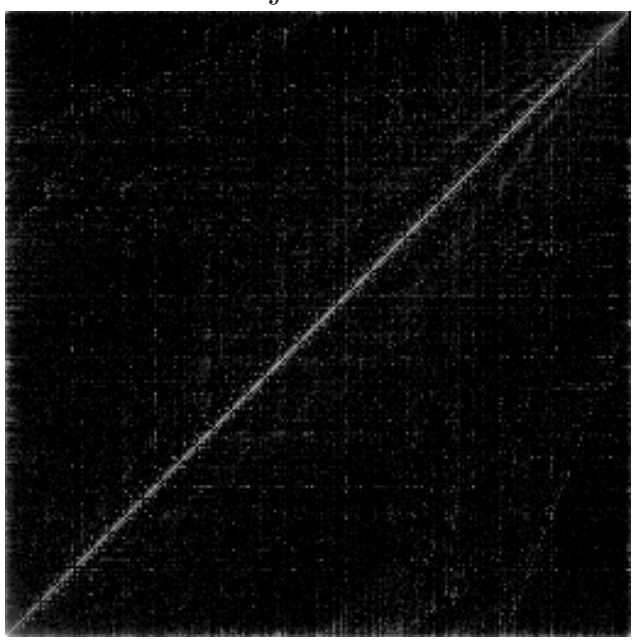

LineArt

Figure 8.7: LUT access images (log normalized) for four types of images. Lighter pixels indicate more accesses to that entry. 


\section{Chapter 9}

\section{Piecewise-Linear Haar}

The Piecewise-Linear Haar (or PLHaar) transform $[43,44]$ is our second contribution to research in $n$-bit to $n$-bit transforms. PLHaar is suitble for lossless and lossy coding, is simple to implement, requires only one lookup table to perform both the forward and inverse transforms, and can compete with the $\mathrm{S}$ and $\mathrm{CFH}$ transforms in terms of PSNR.

\subsection{Transformation by Rotation}

\subsubsection{Haar: an $L_{2}$ Rotation}

Recall from Chapter 6 that the Haar transform is defined by the following equations 9.1 and 9.2 , or in matrix form as in equation 9.3.

$$
\begin{gathered}
H=\frac{B-A}{\sqrt{2}} \\
L=\frac{A+B}{\sqrt{2}} \\
{\left[\begin{array}{l}
L \\
H
\end{array}\right]=\frac{1}{\sqrt{2}}\left[\begin{array}{rr}
1 & 1 \\
-1 & 1
\end{array}\right]\left[\begin{array}{l}
A \\
B
\end{array}\right]}
\end{gathered}
$$

These Haar equations, and the non-normalized Haar transform, transform the domain via a 45-degree (or one-eighth) rotation about the origin in Euclidean, or $L_{2}$, space. That is, each point in the domain can be thought of as lying on a circle whose 

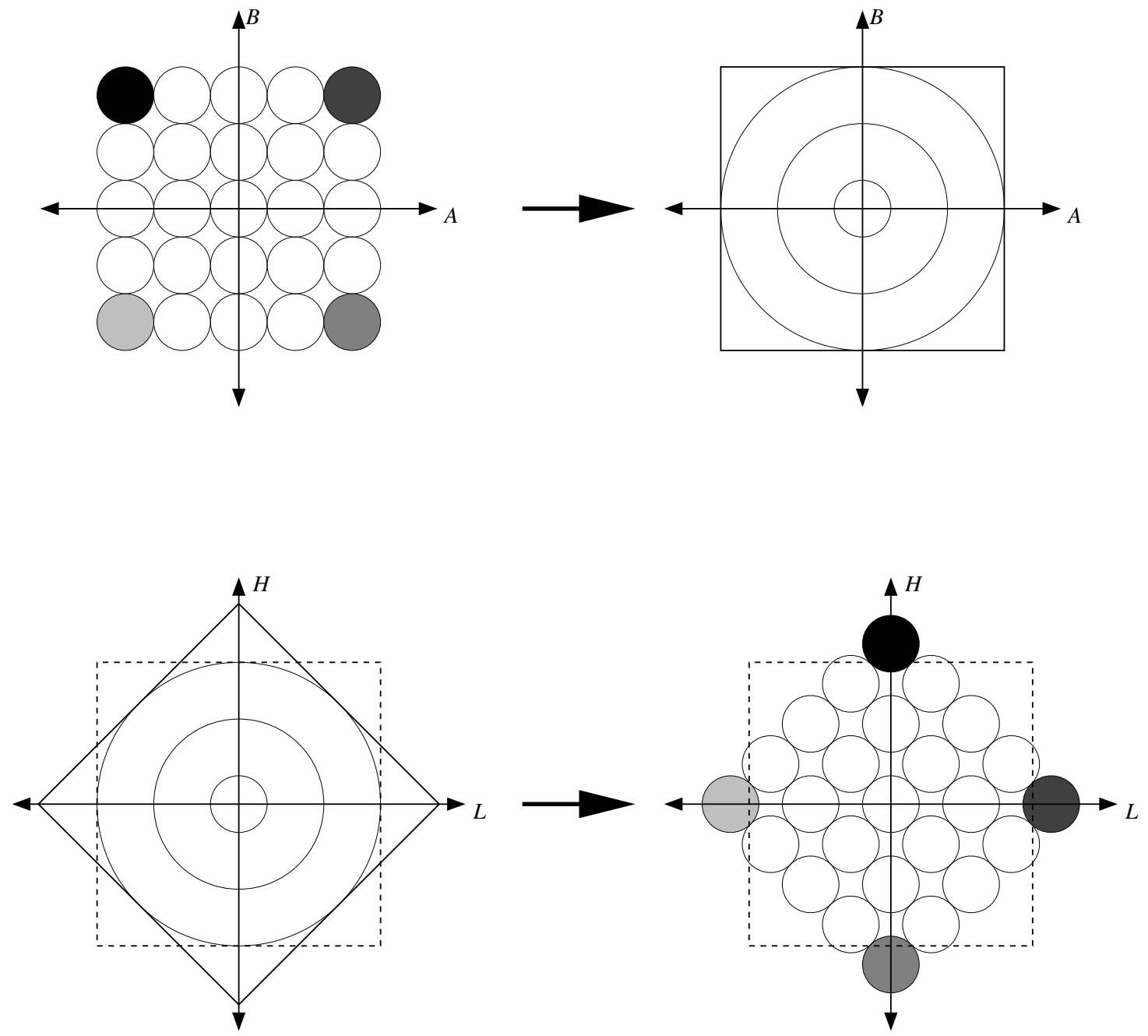

Figure 9.1: Haar $L_{2}$ rotation.

center is at the origin, and whose radius is the distance from the point to the origin. Since the domain is square in shape, the furthest points in the range (the corners of the square) will fall outside the original area occupied by the domain.

\subsubsection{PLHaar: an $L_{\infty}$ Rotation}

The PLHaar transform is a similar rotation, but in $L_{\infty}$ space. In this space, points that are "equidistant" from the origin lie on the perimeter of a square. A one-eighth rotation (analogous to the 45-degree rotation of the Haar transform) about the origin in this space amounts to moving a point one-eighth of the distance along the perimeter of its 

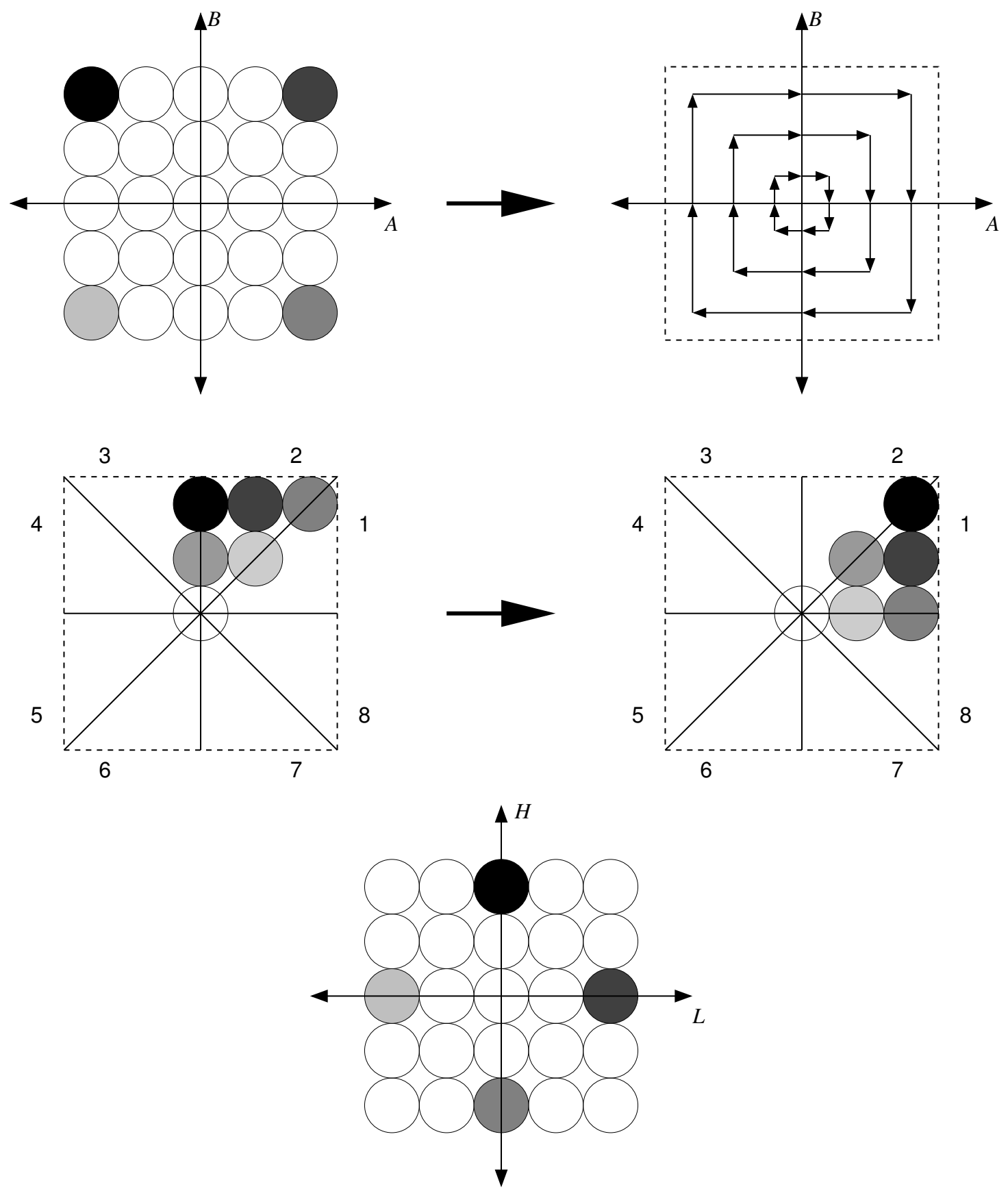

Figure 9.2: PLHaar $L_{\infty}$ rotation. 
square. If we divide the domain and range into octants, then as shown in figure $9.2 \mathrm{a}$ one-eighth rotation moves all points from their positions in a given octant into the next lower octant (with wraparound). The transform as a whole is nonlinear, but when taken on a piecewise (octant-by-octant) basis, the transform from octant to octant is linear. The name "Piecewise-Linear Haar" derives from this property.

The transform of a point at coordinates $(A, B)$ is given by

$$
\left[\begin{array}{l}
L \\
H
\end{array}\right]=R\left[\begin{array}{l}
A \\
B
\end{array}\right]
$$

where $R$ is one of four matrices, as given in equation 9.5, depending on the octant (Oct.) where the point $(A, B)$ is located.

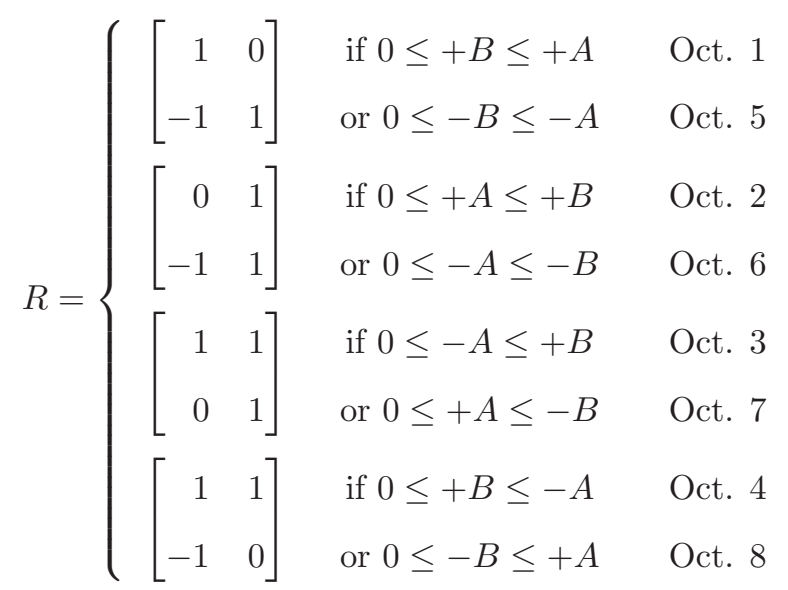

Note that $R$ is the non-normalized Haar matrix, but with one of the four elements zeroed out.

The big advantage of this transform is that it is continuous. Thus, PLHaar is suitable for lossy and lossless coding. Indeed, from the literature it appears that PLHaar is currently the only $n$-bit to $n$-bit transform suitable for lossy coding. Figure 9.3 shows the lookup tables for the S, CFH, PLHaar, and TLHaar transforms. The origin of each table (except for TLHaar) is at its center, with the horizontal axis corresponding to $A$ and the vertical to $B$. Red corresponds to $L$ and green to $H$, biased by 128 . For TLHaar the origin is in the lower-left, and there is no bias. The discontinuities in the CFH and TLHaar transforms were pointed out earlier. The S-Transform has no discontinuities, but 
many of its high-pass entries (as indicated by the blue-tinted areas in the corners) lie outside of the domain.

\subsection{Efficiency Considerations}

In the definitions of the Haar and PLHaar transforms, the choice of using $H=A-B$ or $H=B-A$ is arbitrary; the choices presented thus far make Haar and PLHaar proper rotations. If the opposite choice is made, the result is a rotoinversion $R^{\prime}$-a rotation followed by a reflection - with the property that $R^{\prime}\left(R^{\prime}(A, B)\right)=(A, B)$, i.e. $R^{\prime}=R^{\prime-1}$ is an involution.

Having PLHaar be an involution is a desirable property because it reduces the number of procedures required to compute the forward and inverse transforms - the same procedure is able to compute both. Also, if lookup tables are to be used, instead of a procedure, the same lookup table can be used for the forward and inverse transforms. Thus, compared to other $n$-bit to $n$-bit methods, the storage required for tables is cut in half.

Conceptually, in order to make the transform an involution an additional step is added to the transform, where after rotation $H$ is negated. This causes a vertical flip in the domain. To make PLHaar an involution we negate the second row of each $R$ matrix,

previously defined in equation 9.5. This has no effect on the transform's continuity. If these coefficients are also rotated and flipped, they return to their original positions. This is illustrated for the discrete case in figure 9.4.

\subsection{Characteristics of PLHaar}

\subsubsection{Data Decorrelation}

Wavelet and other transforms are typically used because they provide (among other things) data decorrelation. We see from equation 9.5 that $R(x, x)=(x, 0)$, decorrelating adjacent identical values, just as in the Haar transform. Thus PLHaar should be useful for a data compression system. 

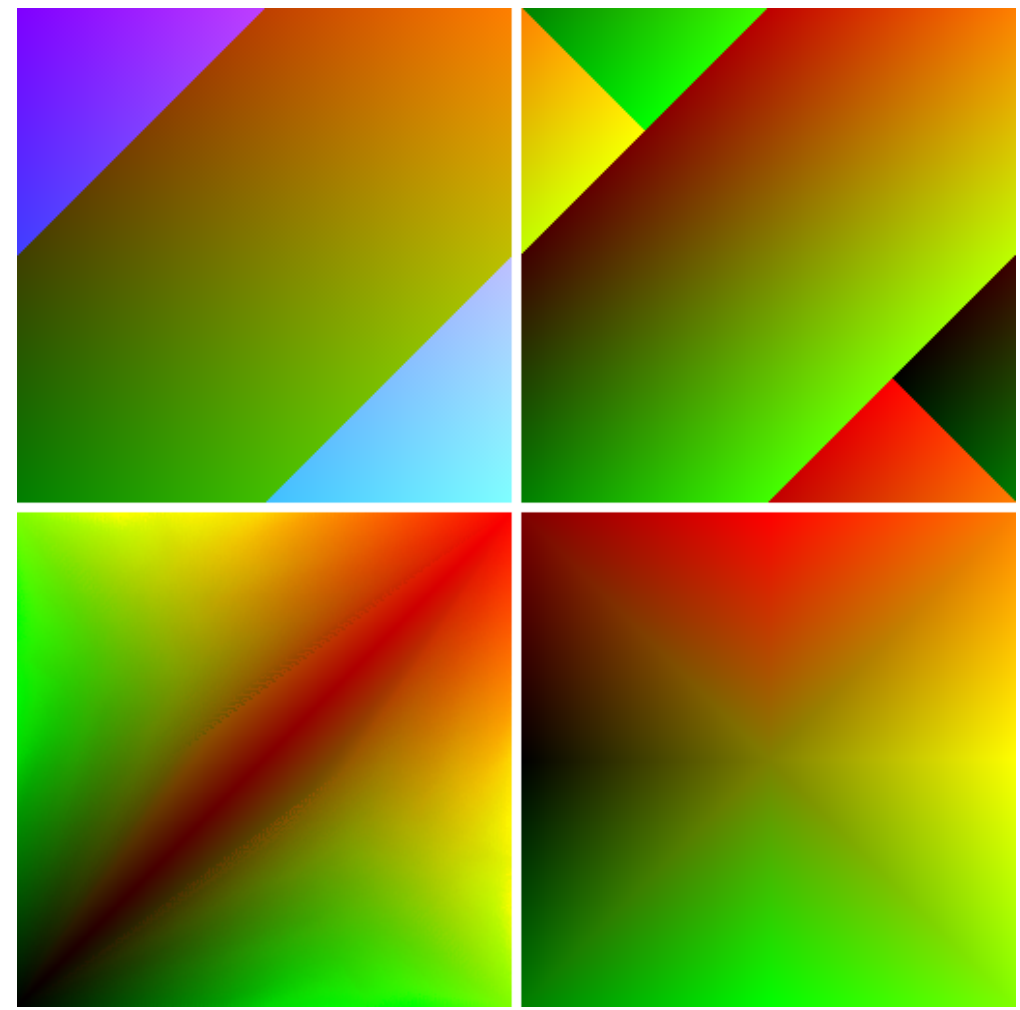

Figure 9.3: The transform LUTs for (clockwise from the upper-left) S-Transform, CFH, PLHaar, and TLHaar. Here we use $A-B$ (and not $B-A$ ) when computing $H$. The PLHaar table is the only one that does not have discontinuities or out-of-range values.

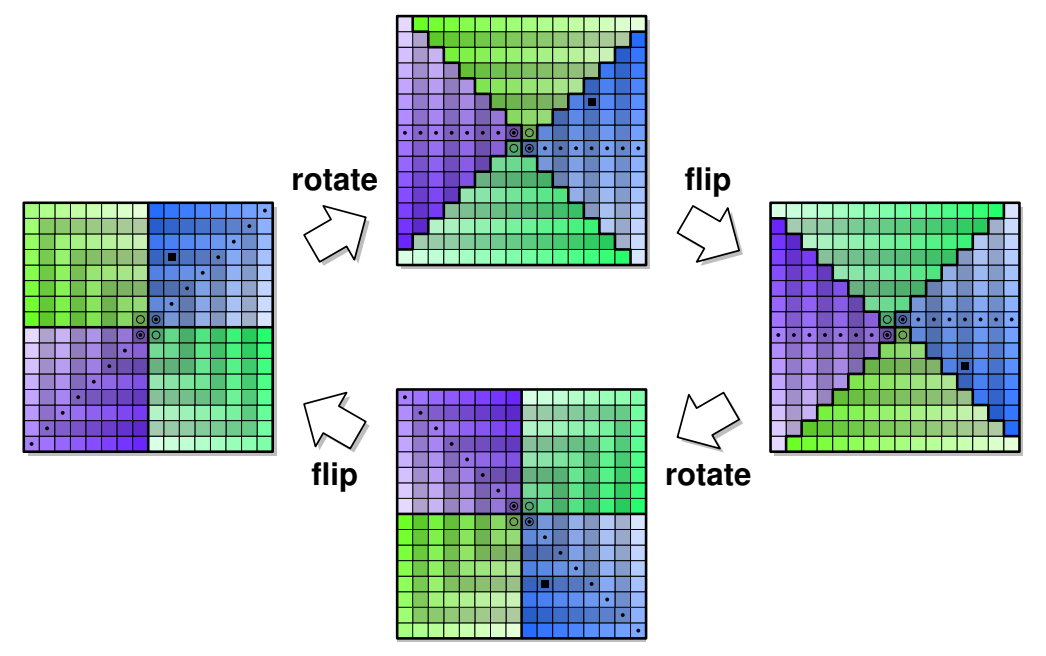

Figure 9.4: PLHaar interpreted as an involution. Note that both rotations are in the same direction. 
To verify that PLHaar is usable as a transform for compression we iteratively transformed some test images, at each iteration applying the transform to the low-pass coefficients resulting from the previous iteration, until there was a single low-pass coefficient remaining. We then took a histogram of the coefficients and measured the normalized zero-order entropy $E[45]$ according to

$$
E=\sum_{i}-p_{i} \times \log _{256} p_{i}
$$

where $p_{i}$ is the probability of coefficient value $i$ and the summation is taken over all nonzero $p_{i}$. We compared the entropy resulting from the PLHaar transform to those of the S, TLHaar, and CFH transforms. Results are in figure 9.5, and we see that in all cases PLHaar results in a reduction in entropy. A comment on the results for the "WashSat" image is in order. The original image had a narrow histogram, and so some form of histogram expansion was performed to enhance contrast. Apparently the $\mathrm{CFH}$ and S transforms do not respond well to this, at least on this particular image - they actually increase the entropy of this image somewhat. A plot of coefficient histograms for the Lena image is given in figure 9.6.

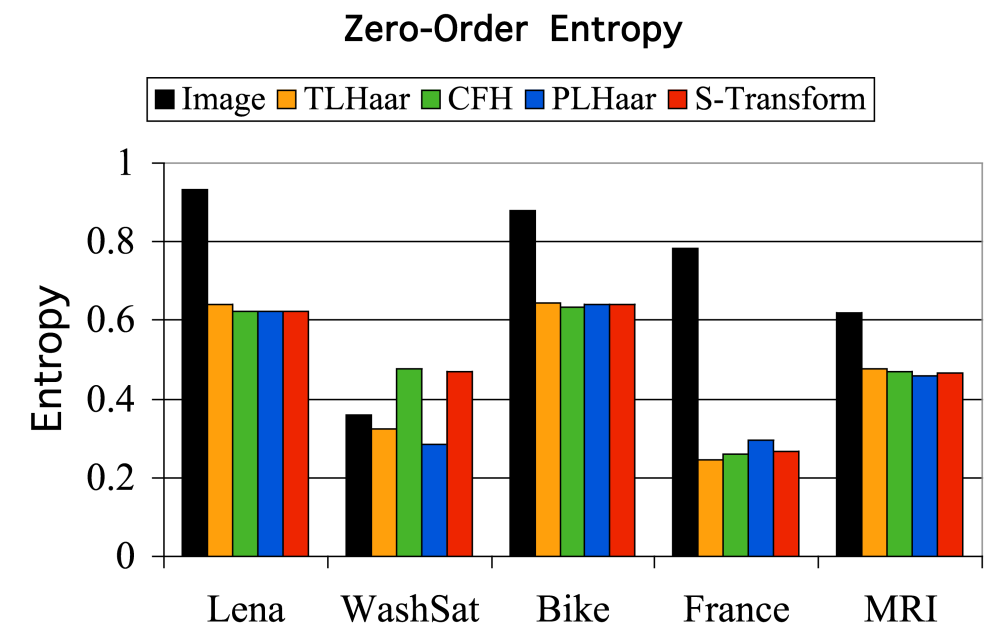

Figure 9.5: Zero-order entropy of the wavelet coefficients produced by the transforms. 


\section{Coefficient Histograms}

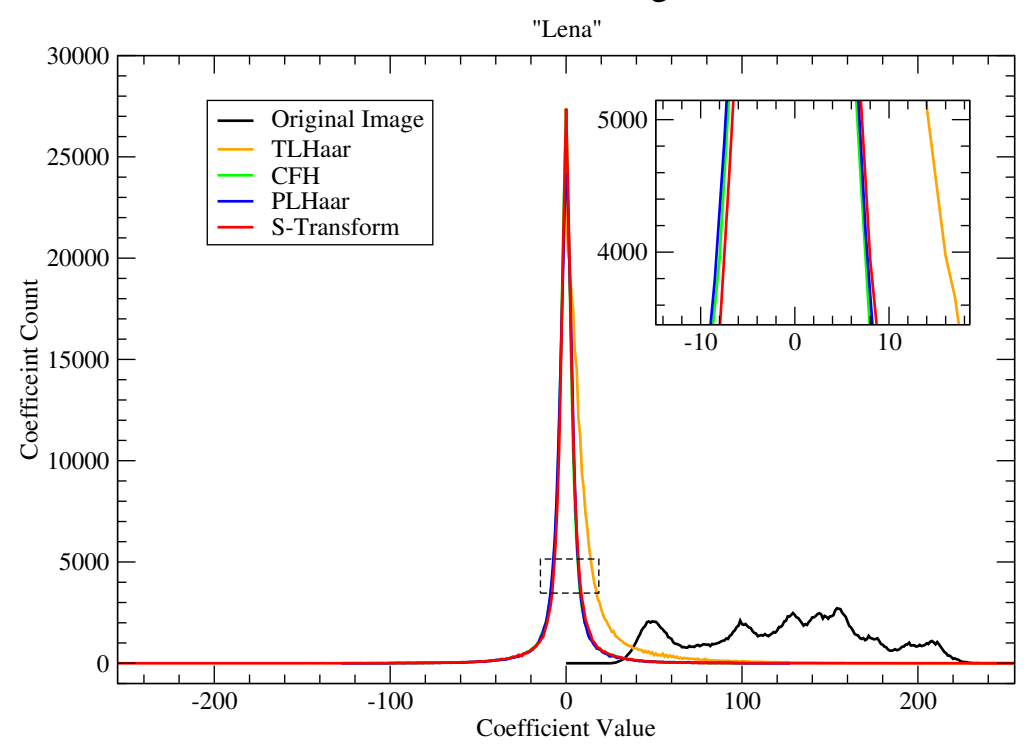

Figure 9.6: Histograms of "Lena" image, and the S-Transform, PLHaar, TLHaar, and $\mathrm{CFH}$ coefficients.

\subsubsection{No Average Preservation}

The next thing that we see about PLHaar is that PLHaar does not preserve averages, while other transforms, such as Haar, CFH, and S, do. The forward and inverse Haar matrices are given in equations 9.7 and 9.8. If we let $A=42$ and $B=50, L=65.05$ and $H=-5.66$. If $H$ is thresholded to zero the reconstructed $A$ and $B$ values are both 46, which is the average of the original $A$ and $B$. If the difference between $A$ and $B$ is great, then the closer $H$ is brought toward zero the closer the reconstructed $A$ and $B$ will be to their average. Thus when an average-preserving transform is used in a multiscale decomposition (such as is commonly done with a wavelet transform) and the amount of coefficient thresholding is significant, the reconstructed image pixels will be closer to the overall average of the image. This causes a kind of "fade to gray" effect, that is illustrated later in figure 9.16.

$$
\left[\begin{array}{l}
L \\
H
\end{array}\right]=\frac{1}{\sqrt{2}}\left[\begin{array}{rr}
1 & 1 \\
-1 & 1
\end{array}\right]\left[\begin{array}{l}
A \\
B
\end{array}\right]
$$




$$
\left[\begin{array}{l}
A \\
B
\end{array}\right]=\frac{1}{\sqrt{2}}\left[\begin{array}{rr}
1 & 1 \\
1 & -1
\end{array}\right]\left[\begin{array}{l}
L \\
H
\end{array}\right]
$$

The forward and inverse transform matrices for the octant 2/6 PLHaar transform (noninvoluted) are given in equations 9.9 and 9.10. If we use the same $A$ and $B$ as before, we compute $L=50$ and $H=8$. If $H$ is set to zero, the reconstructed $A$ and $B$ values are both 50, which is not near the average of the original $A$ and $B$. Instead, it is further away. If PLHaar's coefficients are coded lossily, as more loss is incurred the reconstructed values for $A$ and $B$ will be further from the average, and the reconstructed image will have increased contrast. This has some implications in terms of PSNR; these will be covered later in section 9.5.3.

$$
\begin{aligned}
& {\left[\begin{array}{l}
L \\
H
\end{array}\right]=\left[\begin{array}{rr}
0 & 1 \\
-1 & 1
\end{array}\right]\left[\begin{array}{l}
A \\
B
\end{array}\right]} \\
& {\left[\begin{array}{l}
A \\
B
\end{array}\right]=\left[\begin{array}{lr}
1 & -1 \\
1 & 0
\end{array}\right]\left[\begin{array}{l}
L \\
H
\end{array}\right]}
\end{aligned}
$$

\subsubsection{Signed Zeros}

A rotation must happen about a center point. If a transform is real-valued this is not a concern, as there is always a real-valued center point. If the transform is integer-valued there may not be a center point about which to perform the rotation. Specifically, if the domain has a dimension that is even, there is no integer-valued center to the domain. The solution to this problem is to divide the domain into quadrants, and pack them as shown in figure 9.4. During transformation we select an artificial center: an integer point near the domain's (non-integer) center. Then, each octant is translated so that its corner lies on this center point. The linear transformation of the point is performed, and an inverse translation is performed on the resulting points.

These manipulations are similar (but not equivalent) to performing signed one's complement arithmetic. This presents us with an additional problem. Since the domain had no integer center, the range has no integer center as well. The result is that the 
transform coefficient histogram is not centered about zero, as it is with the Haar, S, and CFH transforms, but about positive and negative zero. The "signs" on the zeros cause difficulties. Since the signs of the zeros are more or less distributed randomly, they must be encoded with 1 bit per pixel (bpp). This means that these signed-zero coefficients cannot be encoded to a bitrate less than 1bpp (There is some work on intelligently encoding wavelet coefficient signs - see [14] for example — but the PSNR improvements in the reconstructed images generally are less than $0.7 \mathrm{~dB}$ ). Since these signs are the least-significant data they can be discarded, but on image reconstruction it is impossible to know which sign a given zero should have.

We refer to PLHaar with signed zeros as PLHaar2, and the unsigned-zero version as PLHaar1. When referring to things common to both transforms we use the term PLHaar.

Note that practically speaking having an even-dimensioned domain may not be an issue. If we are only concerned with keeping the range limited to a size that is equivalent to a standard hardware memory unit (an eight-bit byte, for example) then we may be able to use PLHaar1 on an even domain. For example, if our domain is not 8-bit but fits within 8 bits (a 6 -bit domain, for example, where all values are $<2^{6}$ but each is stored in an 8-bit byte) we can "pad" the domain by assuming it is odd--dimensioned $\left(2^{6}+1\right.$ by $2^{6}+1$ ) and use PLHaar1.

\subsection{Implementation}

Source code for the continuous and discrete PLHaar transforms is given in figures 9.7 and 9.8. Note that both of these procedures implement the modified transform (the involution) described above.

The procedure for the discrete transform uses no extra intermediate precision, and can take both signed and unsigned integers. Its use is self-explanatory, with the exception of the bias parameter, which is used to move the output range. For example, if the inputs are $n$-bit values from a domain $\left[0,2^{n}-1\right]$ the bias parameter should be set to $2^{n-1}$ to keep the high- and low-pass coefficient range equal to the domain. In the 


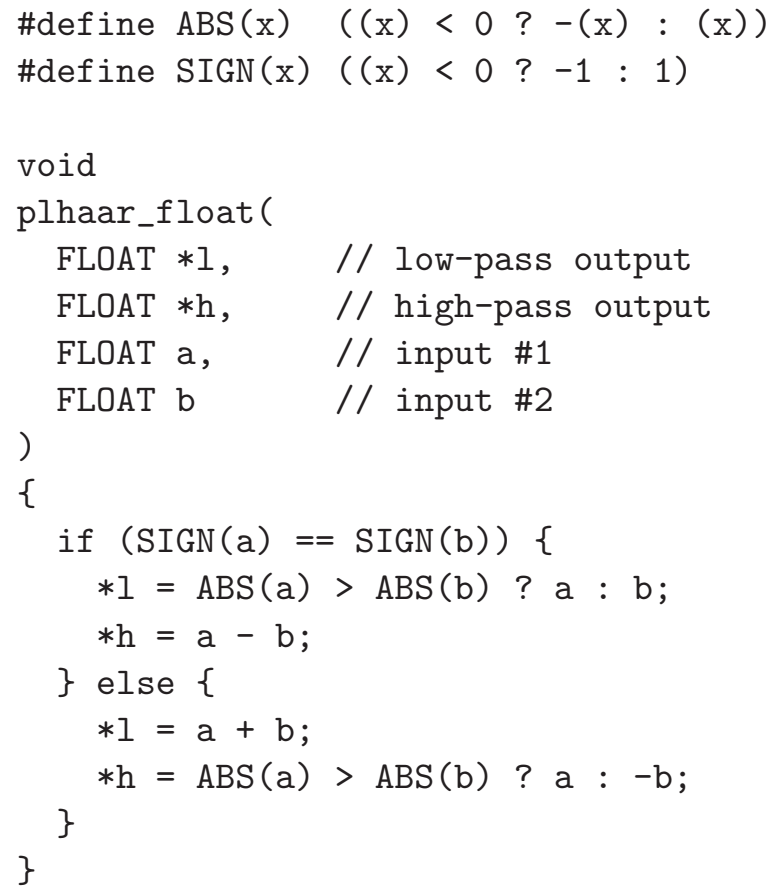

Figure 9.7: Source code for the continuous PLHaar transform.

source, the lines marked $(* *)$ are necessary only when the domain and range contain an even number of integers (e.g. [0,255]) - this is PLHaar2. In this case there is no unique origin, so we translate each quadrant so that its origin is at a common point, perform the transform, then translate the quadrant back. If the domain and range contain an odd number of integers (e.g. $[0,254])$ then the lines marked $(* *)$ may be removed and the bias set accordingly (e.g. 127) - this is PLHaar1.

These procedures are able to perform both the forward and inverse transforms. To perform the inverse transform, $L$ is passed as parameter a, $H$ as $\mathrm{b}$, and $A$ and $B$ are taken respectively from parameters 1 and $h$.

\subsection{Results}

\subsubsection{Execution Time}

To measure execution time we implemented the S, CFH, and PLHaar transforms in both direct-computation and table-lookup versions, and measured the time it took to transform 


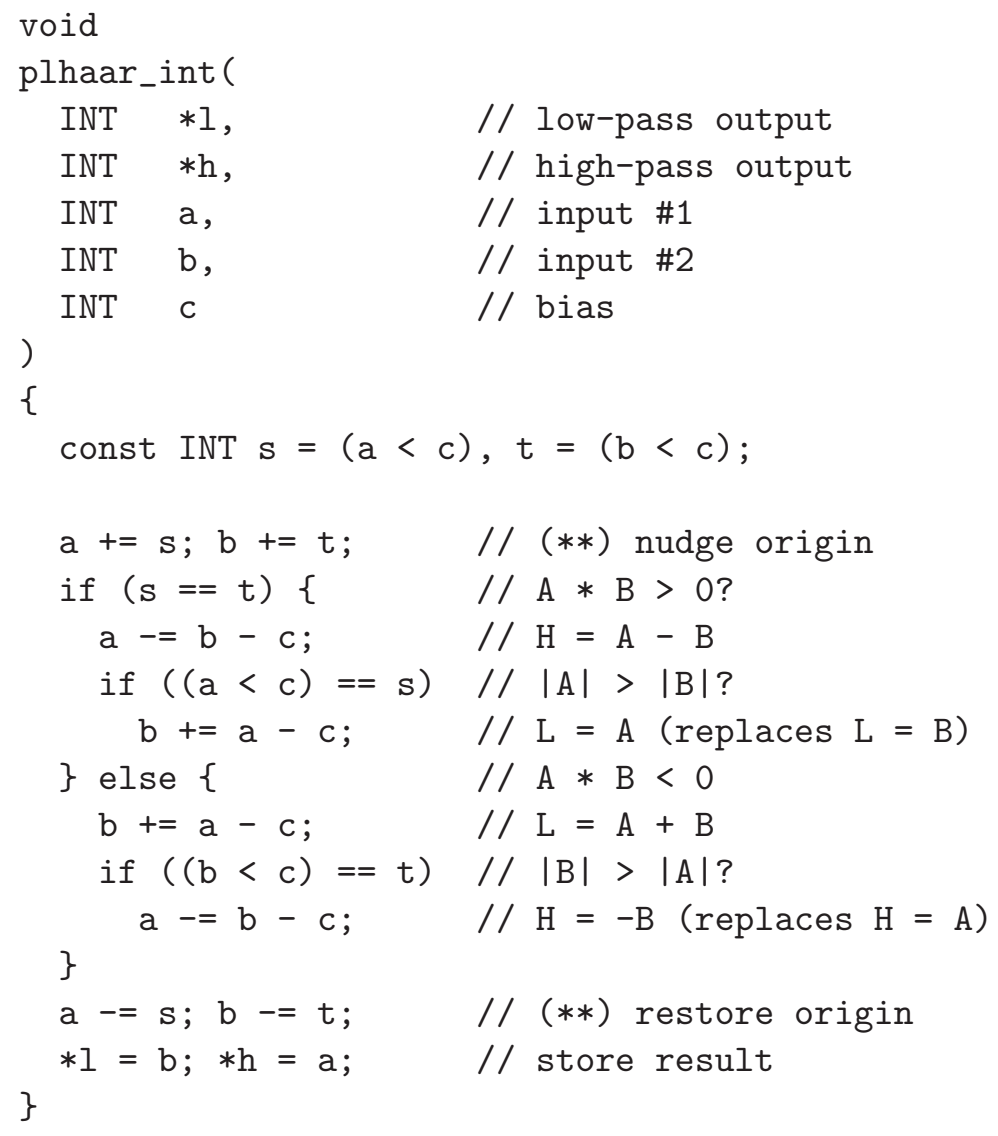

Figure 9.8: Source code for the discrete PLHaar transform. 
square photographic images of edge length 256, 512, 1024, and 2048 pixels.

Because PLHaar is a transform from one octant to the next there is some additional overhead incurred during the transform process: before each point can be transformed its octant must be identified (see equation 9.5). In our current implementation this requires two nested if-then statements. Because of this we anticipated that PLHaar would be slower than the $\mathrm{CFH}$ and $\mathrm{S}$ transforms. We also note that PLHaar execution time may vary, depending on the more frequently taken path through the transform procedure.

Execution time was measured on a Dell Precision Workstation 530 with dual 2.2 GHz Intel Xeon processors (only one was used in the tests), 512 kilobytes of cache, and 512 megabytes of RAM. The operating system was RedHat Linux 3.2.3. All times reported are an average over 256 forward transformations. Results for execution time using direct computation are given in figure 9.9, and for table lookup in figure 9.10. From figure 9.9 we see that as expected the execution times for PLHaar are slower than the S and CFH transforms, due to the additional work required for determining which octant a point is located in. Even so, PLHaar has a throughput of about 28 megabytes a second. When table lookups are used the difference in execution times between the CFH and PLHaar transforms essentially disappears.

Interestingly, for small images the direct computation execution time of the $\mathrm{S}$ transform is slower than the other transforms (this is not obvious from the graphs). We believe that this is because of some additional overhead incurred in the S-transform. Its coefficients are stored in 16-bit short integers, as opposed to 8-bit bytes, and therefore the coefficients do not fit in cache as well as CFH and PLHaar. For larger data this overhead is made up for by the S-transform's fast execution speed.

\subsubsection{Quantization and PSNR}

To gain a basic understanding of how useful PLHaar will be when used in lossy compression or a progressive transmission scheme, we first performed some quantization tests on a set of test images. We transformed the images as in section 9.3.1, and then iteratively quantized the coefficients to shorter bit widths. We treated the S-Transform coefficients as being 


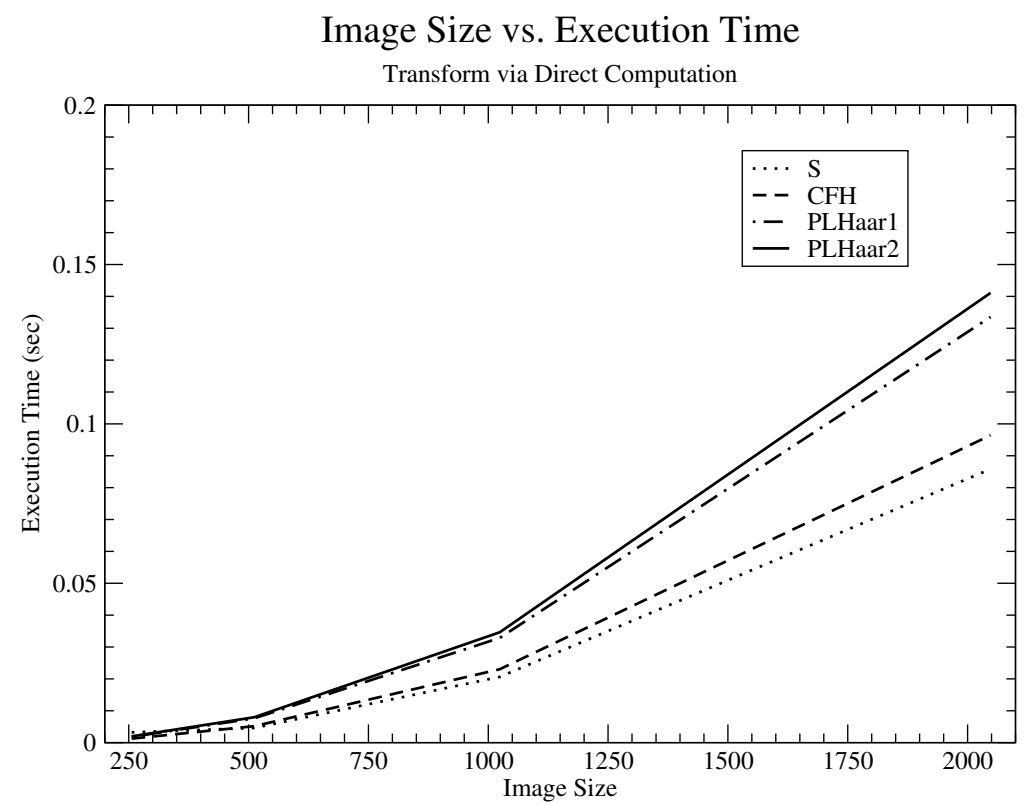

Figure 9.9: Times for direct computation.

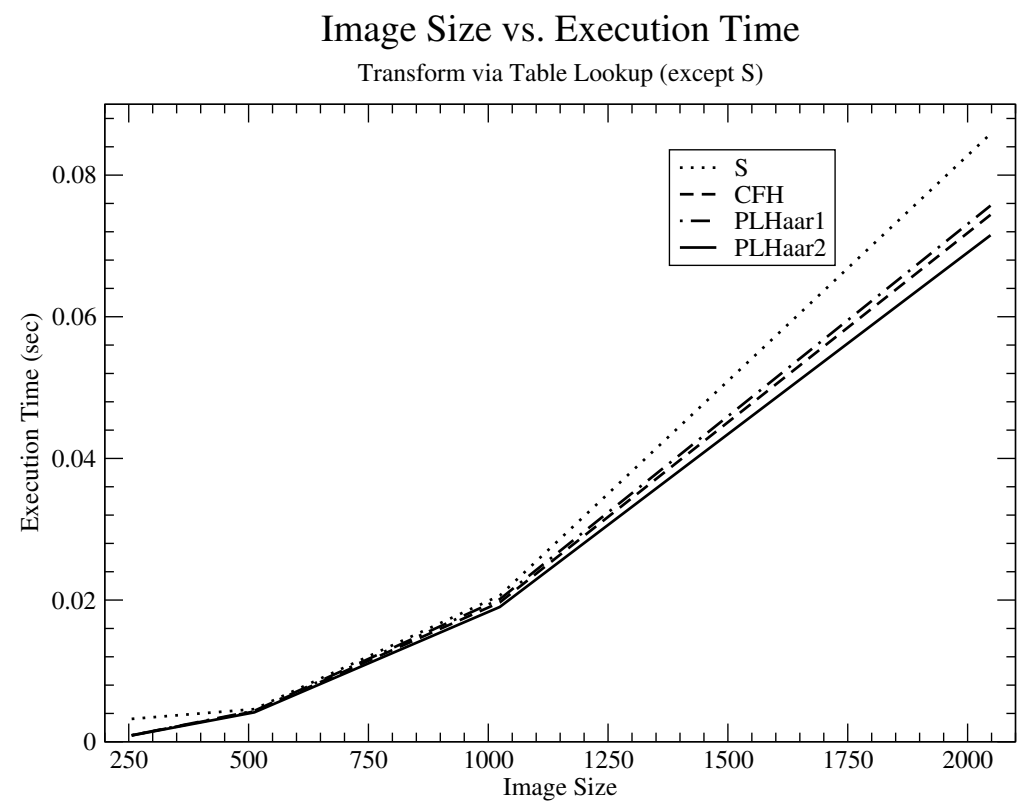

Figure 9.10: Times for table-lookup. 
9 bits wide (sign bit plus an 8-bit magnitude). We used the PLHaar2 transform, and performed sign-preserving quantization on the coefficients.

Quantized coefficients fall into a range of uncertainty. For example, if the coefficient 42 (00101010) is quantized to 5 bits of precision, it falls into the range $[40,47]$. Assuming a uniform distribution, to avoid biasing quantized coefficients towards zero, and to preserve contrast, we place the quantized coefficients near the center of the range of uncertainty. For a given non-negative uncertainty interval $[u, v]$ we compute the center as $\lfloor(u+v) / 2\rfloor$, and center the quantized coefficients accordingly. We then computed the PSNR of the reconstruction.

A graph of the PSNR curves for the "Bike" [6] image is given in figure 9.11, and the $L_{\infty}$ error curves are given in figure 9.12. From these we see that the PLHaar2 transform gives a better reconstruction than all other tested methods. In particular, due to the aliasing problem discussed earlier, the $\mathrm{CFH}$ transform is unable to give a good reconstruction of the image. Figure 9.13 shows the Bike image, transformed by CFH and PLHaar, quantized to 3 bits of precision, and reconstructed. Underneath each image is a set of four details.

As mentioned earlier, the low-pass values computed by PLHaar have a higher contrast than the inputs to the transform. This means that the more PLHaar coefficients are quantized, the higher the contrast in the reconstructed image. Although this increased contrast can result in a lower PSNR, it has the benefit, at a low quantization precision, of helping to bring out edges that are "blurred" away in the CFH and S-Transforms. Figure 9.14 shows the reconstructed "Barbara" image, after transformation by CFH and PLHaar and quantization to three bits. The PSNR of these images is respectively 19.06 and 16.48. An examination of the images shows that the image reconstructed from PLHaar coefficients is visually more appealing. Ignoring the artifacts present in the $\mathrm{CFH}$ reconstruction, we see that the PLHaar reconstruction has preserved such things as the lines in her face (note the eyes), the edges and lines on her pants, and the table covering.

If figure 9.15 we give images of the reconstructed "Lena" image, after being transformed by the TLHaar, CFH, PLHaar, and S transforms and quantized. In the 


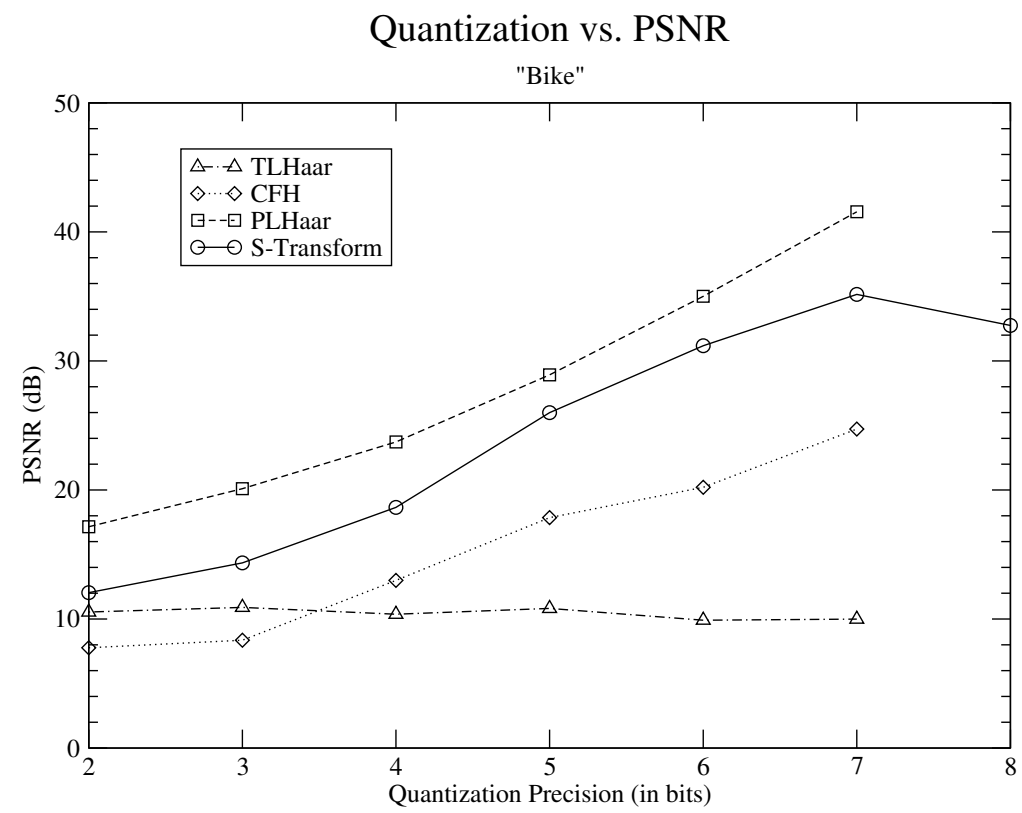

Figure 9.11: Bike: PSNR values for increasing quantization precision. For the $n$-bit to $n$-bit transforms the PSNR values for 8 bits (or no) quantization are not shown, as in this case the PSNR is infinity.

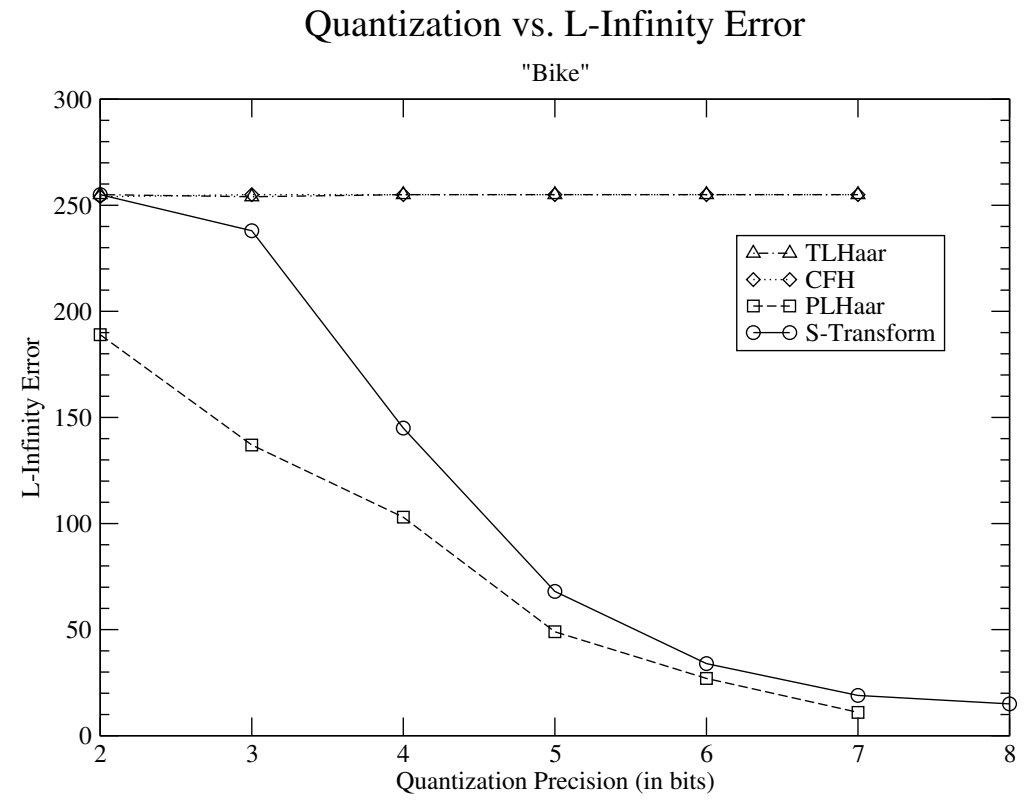

Figure 9.12: Bike: $L_{\infty}$ error values for increasing quantization precision. 


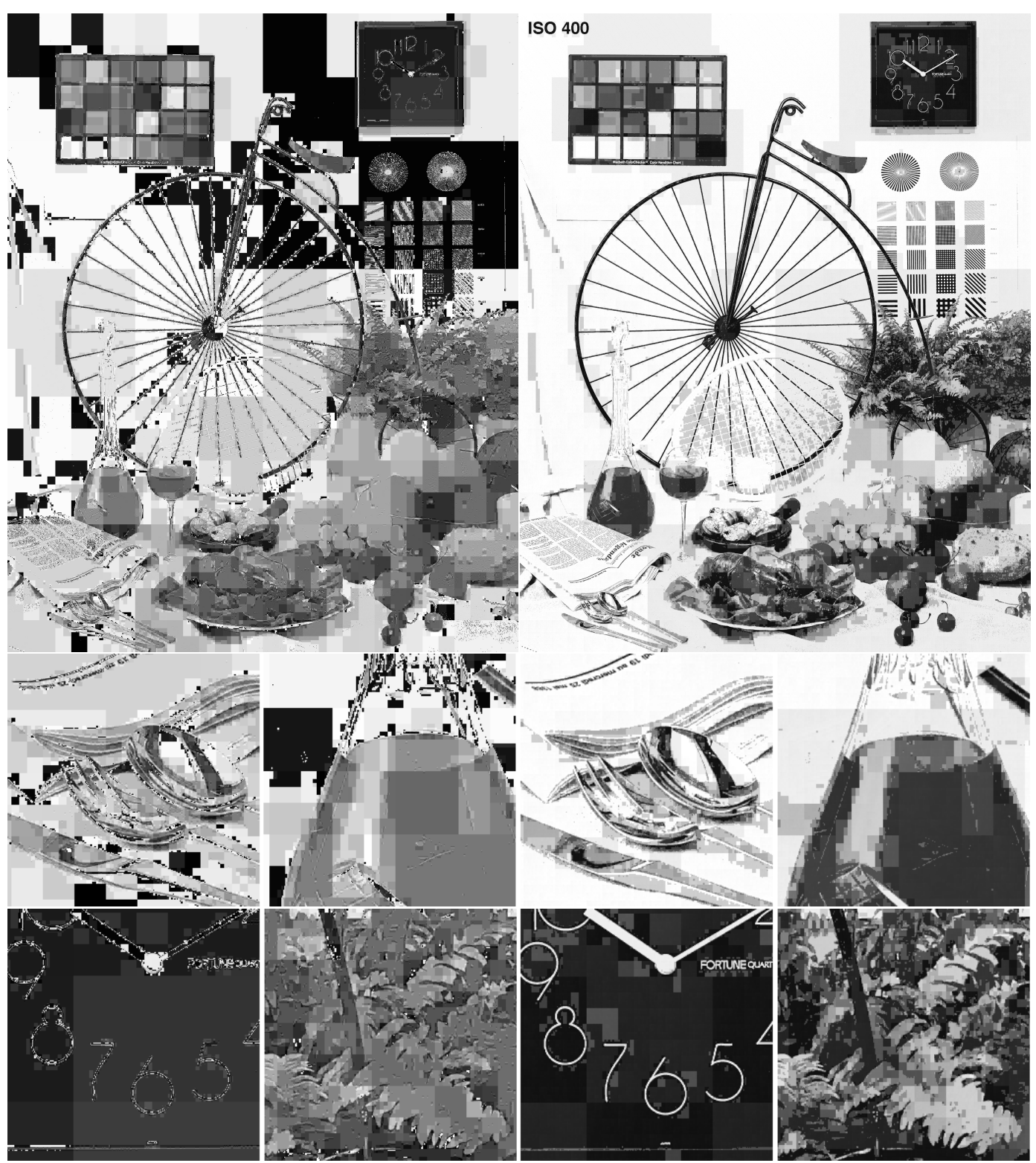

Figure 9.13: Bike [6] image and details, transformed by CFH (left) and PLHaar (right) and quantized to 3 bits. 

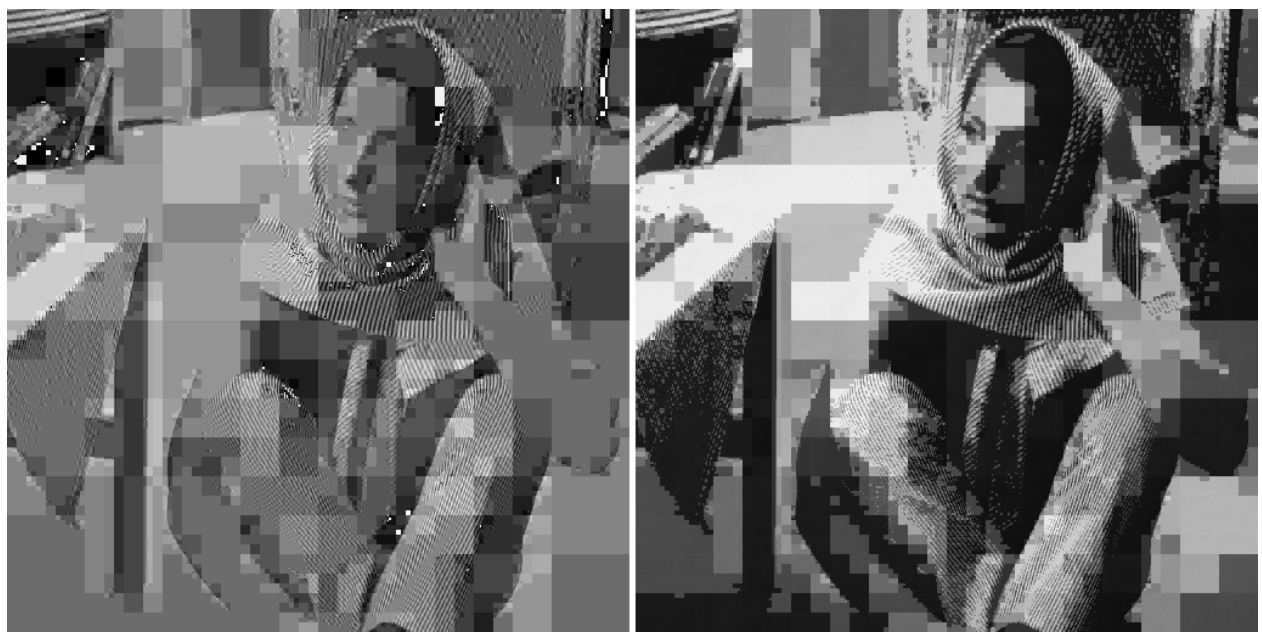

Figure 9.14: Barbara transformed by CFH (left) and PLHaar (right), and quantized to 3 bits.

$\mathrm{CFH}$ reconstruction a few artifacts appear in the three-bit image (note the inner edge of the mirror frame, along the edge of her shoulder, and along the brim of her hat). Also, when comparing the three-bit $\mathrm{CFH}$ and PLHaar reconstructions, we see again how PLHaar's increased contrast improves the visual quality of the reconstructed image. Note for example that in the PLHaar reconstruction her facial features are more intact.

\subsubsection{Thresholding and PSNR}

Thresholding (the zeroing of coefficients less than some threshold $T$ ) is a more common way of lossily coding transform coefficients, so we examine thresholding here. Where PLHaar2 is used we do not preserve coefficient signs, and all thresholded coefficients are set to "negative" zero. Results are given in terms of zero-order entropy vs. PSNR. This allows us to compare PSNR resulting from sets of transform coefficients that would compress to approximately the same size.

Some lossy reconstructions for the Mandrill and Wedding Photo images are given in figures 9.16 and 9.17. From figure 9.17 we see again the artifacts inherent in a CFH reconstruction when the original data has areas of high contrast. The Mandrill image does not contain many areas of high contrast, so in figure 9.16 we see that there are no immediately obvious artifacts in the $\mathrm{CFH}$ reconstruction. We do see that as the bitrate 


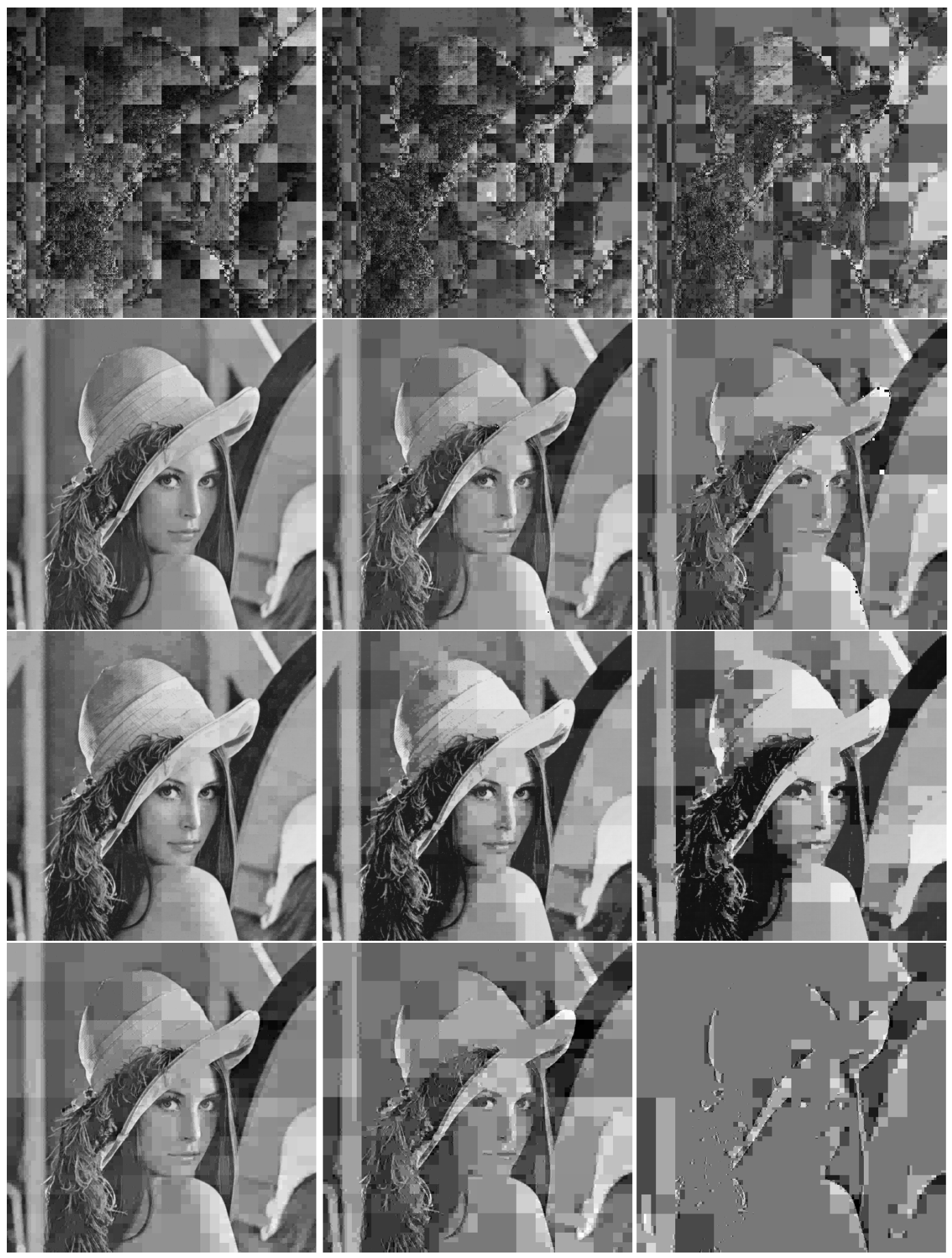

Figure 9.15: Lena, quantized to five, four, and three bits. From the top: TLHaar, CFH, PLHaar, and S-Transform. 


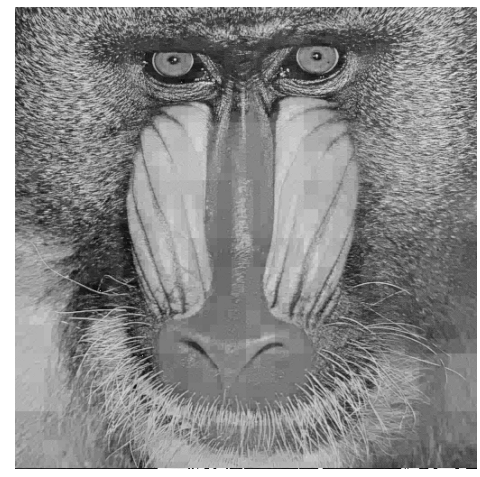

27.82

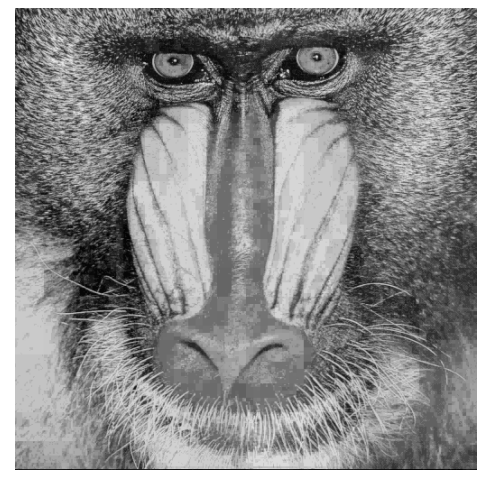

24.82

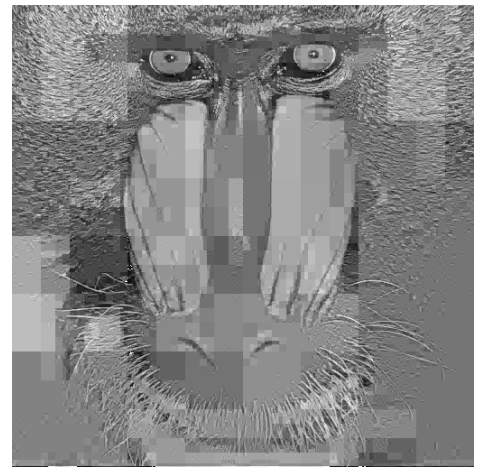

19.86

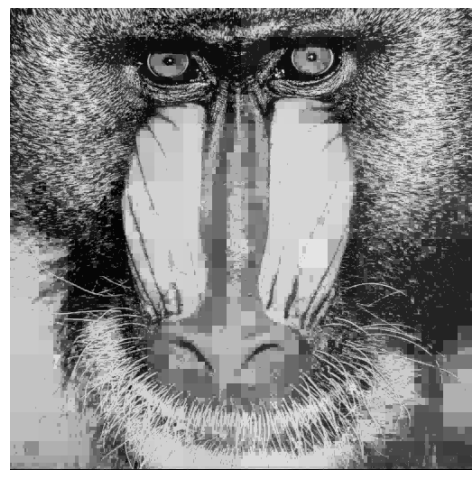

14.42

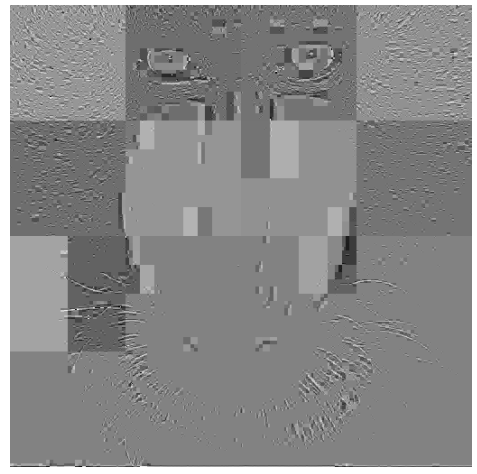

16.93

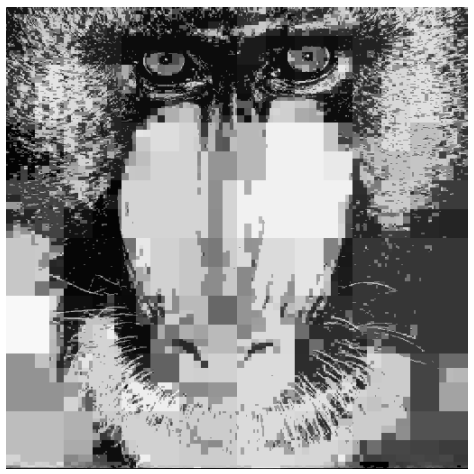

13.85

Figure 9.16: Mandrill, thresholded to an approximate entropy of (from left) four, two, and 0.5 bits per pixel. Upper images are CFH, lower are PLHaar1. PSNR is given beneath each image. 


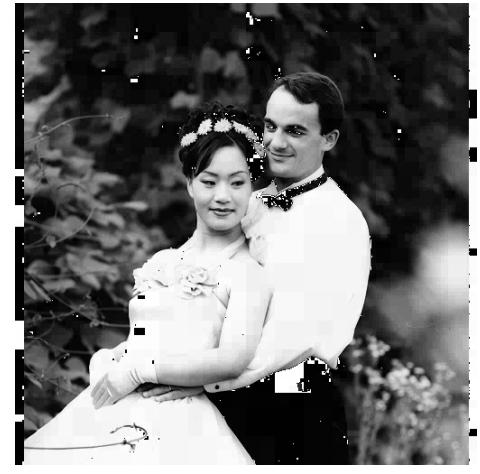

15.92

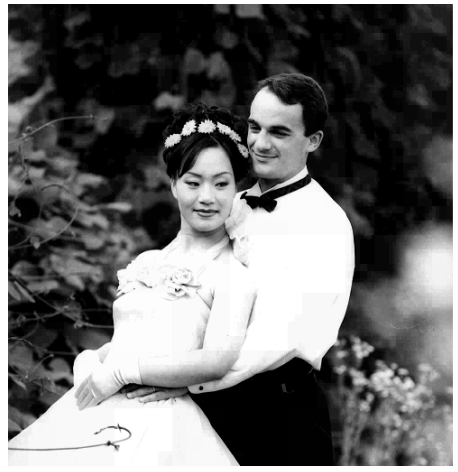

31.32

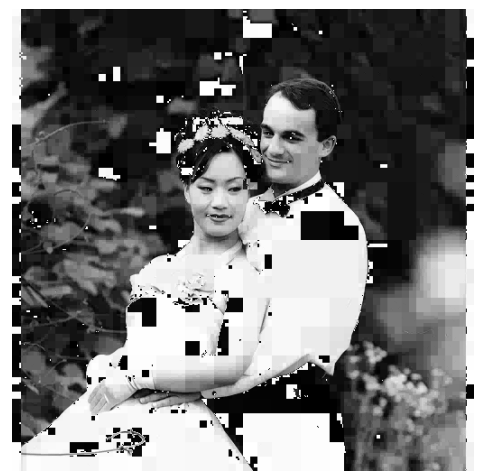

11.21

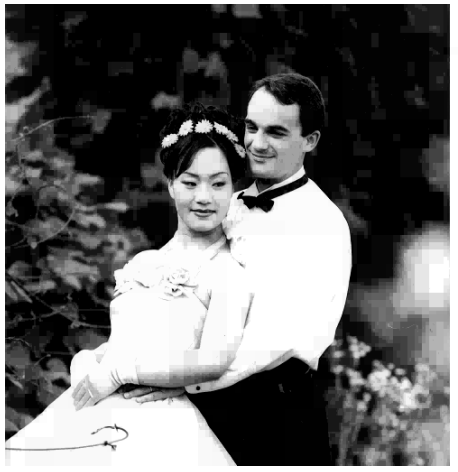

24.54

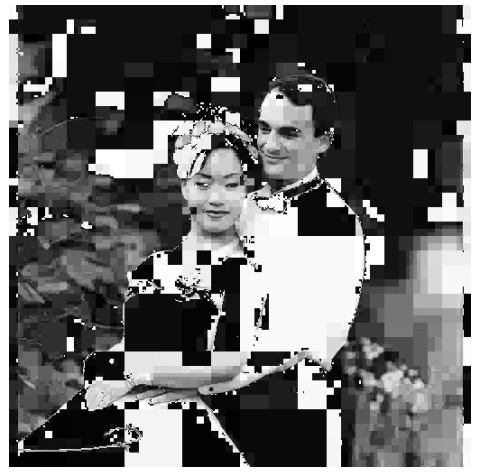

8.04

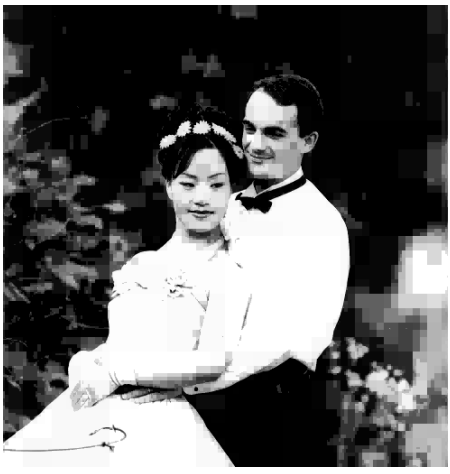

20.27

Figure 9.17: Wedding photo, thresholded to an approximate entropy of (from left) 2.1, 1 , and 0.5 bits per pixel. Upper images are CFH, lower are PLHaar2. PSNR is given beneath each image.

falls the $\mathrm{CFH}$ reconstruction exhibits the characteristic fading (also found in the Haar and $\mathrm{S}$ transforms) as pixel values in the lossy reconstructions are pulled closer to the overall image average. PLHaar does not have this property, so at a lower bitrate the PLHaar reconstruction is more visually appealing — any details in the image are more immediately obvious.

PSNR graphs for four test images are given in figure 9.18. From them we see that for identical zero-entropy values the PLHaar reconstructions have a smaller PSNR than the $\mathrm{S}$ and $\mathrm{CFH}$ transforms. This is due to the increased contrast of the PLHaar reconstruction at a lower bitrate. The PSNR metric favors techniques that produce reconstructions close to the overall average of the original image. Since PLHaar does not do that, its 
PSNR values are lower, although a viewer would most likely indicate that the PLHaar reconstruction was more appealing.

Entropy vs. PSNR, Wedding Photo
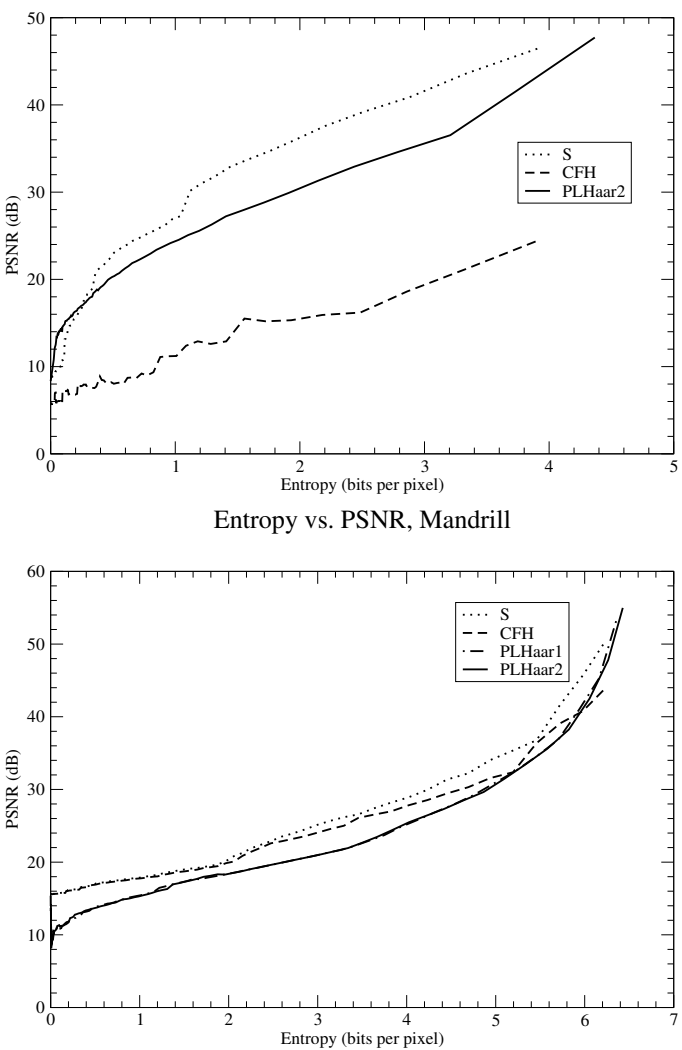

Entropy vs. PSNR, Lena
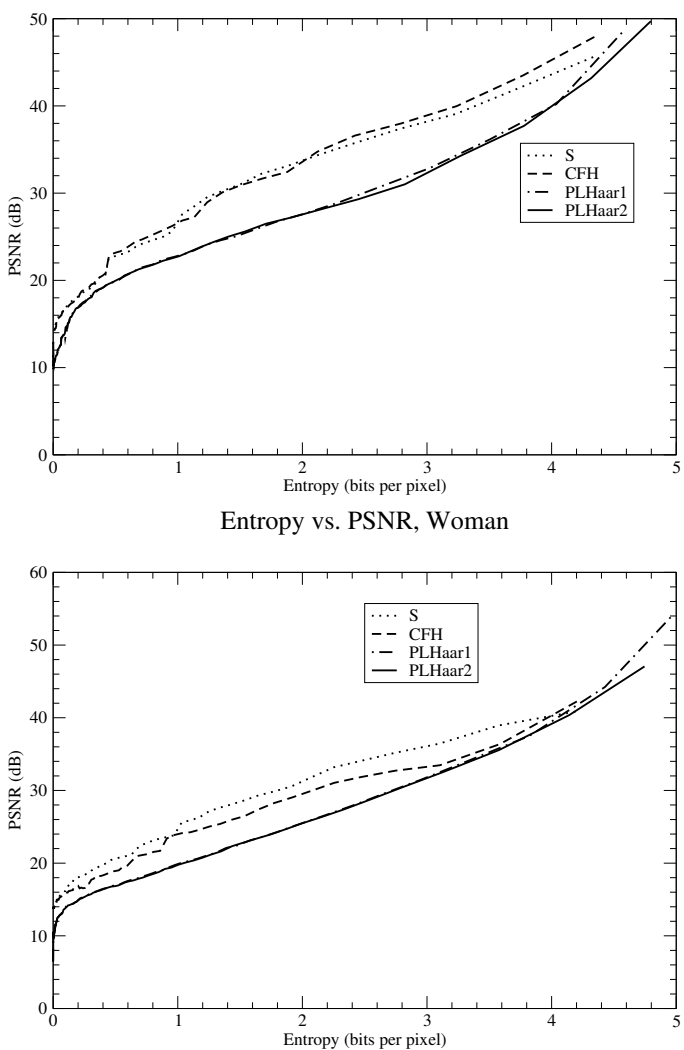

Figure 9.18: PSNR vs. Entropy graphs for four test images.

\subsection{Signed Zeros in PLHaar2}

\subsubsection{Predicting Signed Zeros}

Signed zeros present some difficulties when encoding. Some coding methods, such as those that use zerotrees and similar techniques [34, 26, 25, 38, 46], assume that the coefficients are centered around an unsigned zero. With signed zeros an explicit pass must be made to encode the signs. Also, because these signs are the least-significant parts of the coefficients, we anticipate that in most cases the signs will be fairly randomly distributed, and will not compress well, if at all. 
Naturally, we want to deal with these coefficients as efficiently as possible. Ideally, we would like to see if there is some correlation between the sign of a zero and some other quantity. There are two types of correlation to exploit: parent-child (PC) correlation, and $\mathrm{H}-\mathrm{L}$ pair (HL) correlation. In PC correlation we attempt to see if there is any correlation between the sign of a zero high-pass coefficient and the sign of its parent in the wavelet tree. In HL correlation we attempt to see if there is a correlation between the sign of a zero high-pass coefficient and the sign of its associated low-pass coefficient.

HL correlation would allow us to not encode the signs of the zero coefficients, and then during image reconstruction set the sign of the coefficient to be the same as that of its associated low-pass coefficient.

To measure the amount of sign correlation in the PC and HL relationship we transformed several representative images from our suite of test images. Results are shown in figure 9.19. In this figure a value of 50 indicates no correlation, and 100 indicates perfect correlation. From this we see that the highest levels of correlation are found in images that have large regions of a single shade, such as line art and MRI images (which are padded to a dimension of $256 \times 256$ pixels). Overall, there does not appear to be any consistently exploitable sign correlation. This is not good, because it means that for an image to be coded losslessly the signs will have to be coded explicitly, thereby hurting the execution time and coding rate.

However, the signs of the zeros are the least-important information in the transform coefficients. One good consequence of this lack of sign correlation is that if an image is to be used for something not requiring perfect reconstruction - as are probably most uses (e.g. viewing on the Internet, photo proofing, textures in games, etc.) - then the signs can be thrown away without any visible decline in image quality. Any "reasonable" scheme can be used to add the signs to the coefficients when the image is reconstructed.

\subsubsection{Signed Zeros and PSNR}

Here we briefly examine the signed zeros in PLHaar2 and their effect on PSNR. To do this we implemented PLHaar2 with thresholding when signs are preserved and not preserved. 
- PC Zero $\mathbb{Q}$ HL Zero

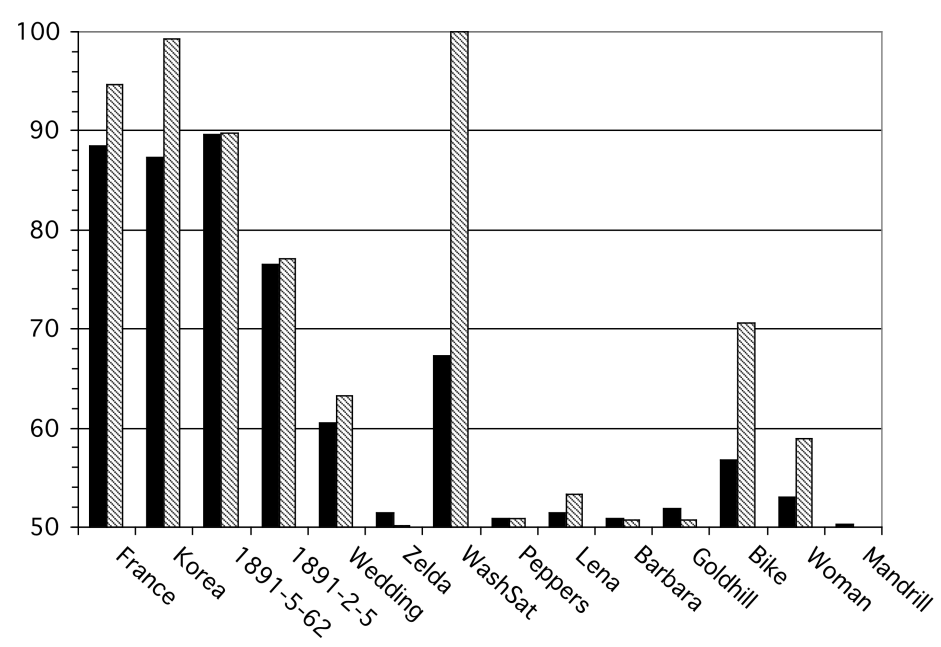

Figure 9.19: Amount of zero sign correlation for PC and HL coefficient relationships. A value of 50 indicates no correlation, and 100 indicates perfect correlation.

In the case of not preserving the signs they are all set to "negative" zero. We then used LH correlation to predict the signs of the zeros when signs are not preserved. If a high-pass coefficient is zero it is given the sign of its corresponding low-pass coefficient.

As would be expected from figure 9.19 the results are not very good. Figure 9.20 gives the Entropy vs PSNR plots of the four test images from figure 9.18. In these plots +/-Pres indicates if the zero coefficients' signs were preserved, and +/-Pred indicates if sign prediction was performed. We see from the figures that the signs are not very predictable. Other tests, not reported here, indicate that if the zero coefficient sign bits are not preserved, then there is no sure method for reconstructing the signs. Setting them to all negative or positive, or initializing randomly, does not deliver consistent results. One positive conclusion that we can draw from the figures is that since the signs are the least-significant data in the coefficients it does not make much difference what we do with them. 

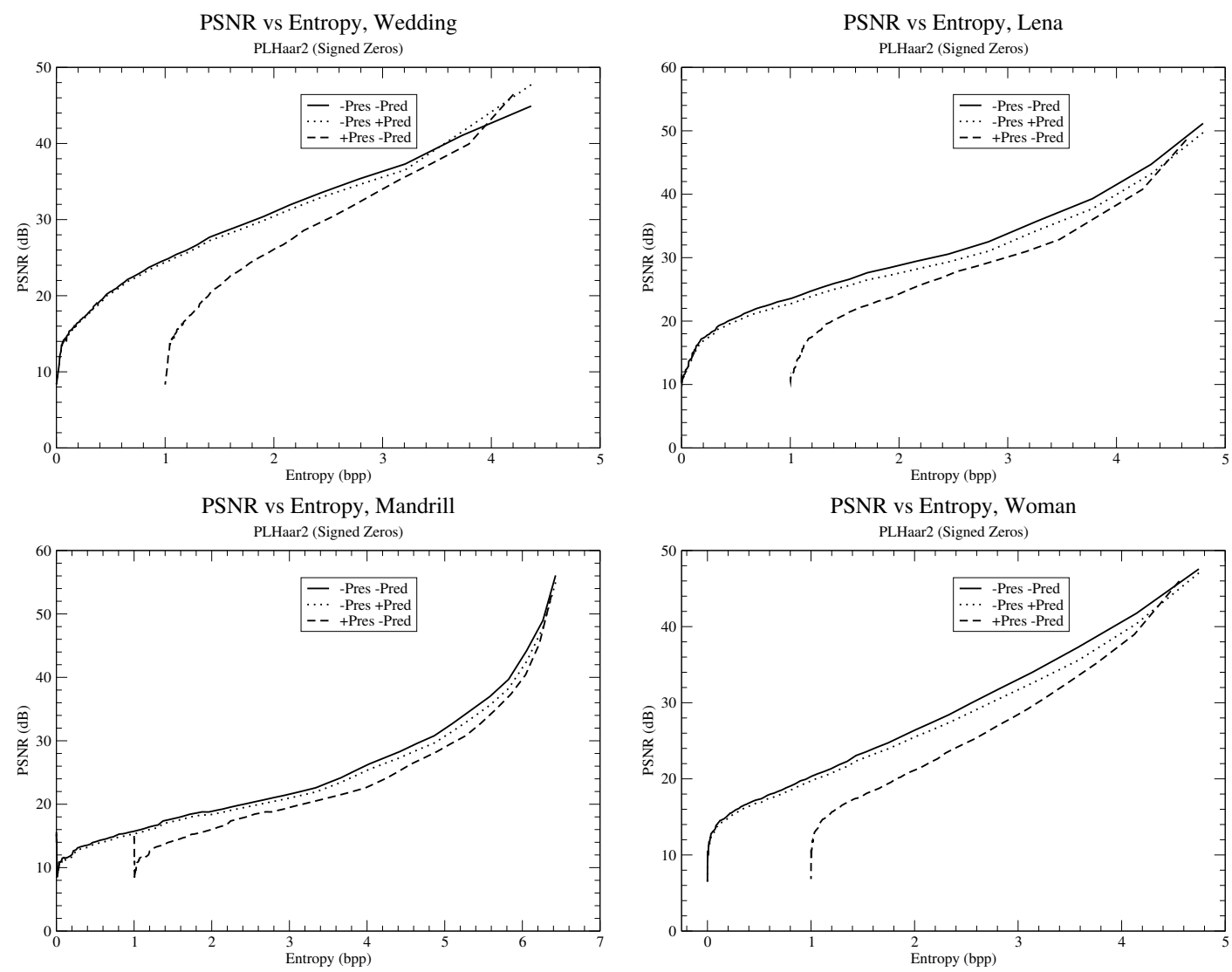

Figure 9.20: PSNR vs. Entropy graphs for four test images using PLHaar2. 


\subsection{Conclusions and Possible Extensions}

To our knowledge the Piecewise-Linear Haar transform is the first transform to demonstrate that it is possible to eliminate dynamic range expansion and have a transform useful for lossy and lossless coding. Eliminating DRE and remaining useful for lossy and lossless coding comes at a price, the major one being that when performing the transform by direct computation PLHaar is slower than other transforms, although table lookup execution is slightly, but consistently, faster than other transforms. Another issue is PLHaar's increased contrast at lower bitrates. Whether this is good or bad depends entirely on the user's requirements. While increased contrast isn't favored by the PSNR metric, it is more likely to satisfy the casual viewer.

For the future there are many things that need to be investigated. The first question is, now that we have successfully created a useful $n$-bit to $n$-bit transform that approximates the simple Haar transform, can we move on and create $n$-bit to $n$-bit transforms that are more complex, using a lifting scheme for instance?

PLHaar has increased contrast at lower bitrates. While this is good for the viewer, image metrics do not favor it. If we desire to "play the metric game" to improve PSNR what changes need to be made to the transform?

PLHaar was developed with the intent of using it in hardware. We need to write a version for execution on modern graphics hardware, and perhaps even prototype some other custom hardware using FPGA logic.

Another matter we need to address is the theory behind $n$-bit to $n$-bit transforms. While Chao, Fisher, and Hua demonstrated $n$-bit to $n$-bit transforms using modular arithmetic, we showed that this method is not suitable for lossy coding. What can we say about $n$-bit to $n$-bit transforms that are good for lossy coding? Are more complex (and better) transforms even possible? If so, what are they? Do they exist but are impractical for use and implementation? Or is the PLHaar transform the best we can do? Any definite theoretical work in this area would be a significant contribution to the art. 


\section{Chapter 10}

\section{Conclusions and Future Directions}

This dissertation presents several solutions for performing data compression and transformation in length-limited environments.

Chapters 4 and 5 described solutions for the problem of requiring length-limited codes. These were respectively variable-to-variable length codes, and Lead-1 encoding for the extraction of isosurfaces from time-varying volume data. We created VV codes through a merging process, as opposed to the prevailing extension process, and demonstrated that a coder using these codes can compete - in terms of compression rate and speed - with other state-of-the-art coding techniques. We showed that Lead-1 encoding, while simple, provides an effective and fast method of encoding transform coefficients. These methods, and the results derived from using them, are a reminder that despite the plethora of sophisticated data compression techniques available, sometimes the best solution is one that is both simple and well thought out.

Chapters 8 and 9 present two techniques for performing a Haar-like transform without dynamic range expansion: the Table-Lookup Haar and Piecewise-Linear Haar transforms. With the PLHaar transform in particular we demonstrate that it is possible to have a transform with no dynamic range expansion that is also simple, compact, fast, and suitable for lossy and lossless coding. PLHaar is the first of its kind, to our knowledge.

There is quite a lot of work to be done, particularly in developing transforms with no dynamic range expansion. Of particular interest is the theory behind such transforms. 
If no dynamic range expansion occurs during the transform then a tradeoff occurs. This tradeoff can take the form of a loss of continuity (in the case of the $\mathrm{CFH}$ transform) or a slower execution speed (in the case of the PLHaar transform). What can we say about transforms with no dynamic range expansion? Exactly what are the tradeoffs? Can more complex, continuous transforms be created, or is it impossible? Some concrete theory surrounding these issues is needed.

With regards to the bin coder, to make such a coder more practical it would be beneficial to develop a system where the design of the coder (number of bins, specific code used for each bin, etc.) is automated: the user specifies a few parameters, and the rest is automatic. Such a system is likely to involve conflicting requirements (e.g. a high compression rate but a low number of bins), and it would be both fascinating and beneficial to develop a systematic approach for negotiating between them. Also, a study of bin output interleaving would be a worthwhile contribution. A system where bin outputs are interleaved with a dynamic granularity could potentially lead to some significant improvements in coder throughput. 


\section{Bibliography}

[1] The USC-SIPI image database. http://sipi.usc.edu/services/database/.

[2] USF flash mob supercomputer home page. http://www.flashmobcomputing.org/.

[3] Virginia tech terascale computing facility. http://www.tcf.vt.edu/.

[4] Vision list imagery archive. ftp://ftp.vislist.com/IMAGERY/MED_3D_SLICES.

[5] Waterloo bragzone. http://links.uwaterloo.ca/bragzone.base.html.

[6] ISO 12640. Graphic Technology-prepress digital data exchange-standard color image data (scid), 1995.

[7] Fingerprint verification contest 2000. http://bias.csr.unibo.it/fvc2000/, 2000.

[8] Martin P. Boliek, James D. Allen, Edward L. Schwartz, and Michael J. Gormish. Very high speed entropy coding. In IEEE International Conference on Image Processing, pages 625-629, 1994.

[9] Leon Bottou, Paul G. Howard, and Yoshua Bengio. The Z-coder adaptive binary coder. In Proceedings DCC '98 Data Compression Conference, pages 13-22, 1998.

[10] J.A. Briffa and V. Buttigieg. Error performance of Tunstall coding. Electronics Letters, 35(23):2021-2022, Nov 1999.

[11] Johann A. Briffa. Investigation of the error performance of Tunstall coding. B.Eng.(Hons.) Final Year Dissertation, University of Malta, Faculty of Engineering, 1997.

[12] Hongyang Chao and Paul Fisher. An approach to fast integer reversible wavelet transforms for image compression, 1996.

[13] Honyang Chao, Paul Fisher, and Zeyi Hua. An approach to integer wavelet transformations for lossless image compression. In Chen, Li, Micchelli, and $\mathrm{Xu}$, editors, Advances in Computational Mathematics, volume 202 of Lecture Notes In Pure and Applied Mathematics, chapter 2, pages 13-38. Marcel Dekker, Inc., 1999.

[14] Aaron Deever and Sheila S. Hemami. What's your sign? Efficient sign coding for embedded wavelet image coding. In Data Compression Conference, pages 273-282, 2000 .

[15] Peter Deutsch and Jean-Loup Gailly. ZLIB specifications. http://www.zlib.net/ zlib_docs.html. 
[16] Francesco Fabris. Variable-length-to-variable-length source coding: A greedy stepby-step algorithm. IEEE Transactions on Information Theory, 38(5):1609-1617, 1992.

[17] Francesco Fabris and Andrea Sgarro. On the composition of Tunstall messages. IEEE Transactions on Information Theory, 45(5):1608-1612, Jul 1999.

[18] G.H. Freeman. Divergence and the construction of variable-to-variable-length lossless codes by source-word extensions. In DCC '93: Data Compression Conference, pages 79-88, 1993.

[19] S. W. Golomb. Run-length encodings. IEEE Trans. Inform. Theory, IT-12:399-401, Jul 1966.

[20] R. C. Gonzalez and R. E. Woods. Digital Image Processing. Addison-Wesley, Boston, MA, 1992.

[21] B. Gregorski, J. Senecal, M.A. Duchaineau, and K.I .Joy. Adaptive extraction of timevarying isosurfaces. IEEE Transactions on Visualization and Computer Graphics, 10(6):683-694, 2004.

[22] Benjamin F. Gregorski. Multiresolution Tetrahedral Meshes for Scientific Visualization. PhD thesis, Computer Science Department, University of California, Davis, June 2004.

[23] Digital Display Working Group. Digital Visual Interface, revision 1.0 edition, April 1999.

[24] V.K. Heer and H-E Reinfelder. A comparison of reversible methods for data compression. Medical Imaging IV, Proc SPIE 1233, pages 354-365, 1990.

[25] Edwin S. Hong and Richard E. Ladner. Group testing for image compression. In J. Storer, editor, Proceedings DCC 2000 Data Compression Conference, pages 3-12. IEEE Computer Society, 2000.

[26] Edwin S. Hong and Richard E. Ladner. Group testing for image compression. IEEE Transactions on Image Processing, 11(8):901-911, Aug 2002.

[27] David A. Huffman. A method for the construction of minimum-redundancy codes. Proceedings of the IRE, 40(9):1098-1101, 1952.

[28] Joint Photographic Experts Group. JPEG Homepage. http://www.jpeg.org/jpeg/ index.html.

[29] A. Kiely and M. Klimesh. A new entropy coding technique for data compression. Technical Report IPN 42-146, Jet Propulsion Laboratory-CIT, 2001.

[30] Aaron Kiely and Matthew Klimesh. An adaptable binary entropy coder. In Proceedings DCC 2001: Data Compression Conference, pages 391-400. IEEE Computer Society, 2001. 
[31] Aaron Kiely and Matthew Klimesh. The ICER progressive wavelet image compressor. The Interplanetary Network Progress Report 42-155, July-September 2003, Jet Propulsion Laboratory, pages 1-46, Nov 2003.

[32] Lawrence Larmore and Daniel Hirschberg. A fast algorithm for optimal length-limited Huffman codes. Journal of the Association for Computing Machinery, 37(3):464-473, Jul 1990.

[33] A. A. Mirin, R. H. Cohen, B. C. Curtis, W. P. Dannevik, A. M. Dimits, M. A. Duchaineau, D. E. Eliason, D. R. Schikore, S. E. Anderson, D. H. Porter, P. R. Woodward, L. J. Shieh, and S. W. White. Very high resolution simulation of compressible turbulence on the IBM-SP system. In Proceedings of the 1999 ACM/IEEE conference on Supercomputing, November 1999.

[34] Tanzeem Muzaffar and Tae-Sun Choi. Wavelet based image compression using subband threshold. In Proceedings of Spie - the International Society for Optical Engineering, volume 4790, pages 491-498. SPIE-International Society of Optical Engineers, 2002.

[35] F. Ono, S. Kino, M. Yoshida, and T. Kimura. Bi-level image coding with melcode comparison of block type code and arithmetic type code. In GLOBECOM '89. IEEE Global Telecommunications Conference and Exhibition. Communications Technology for the 1990s and Beyond, pages 255-260, 1989.

[36] W. B. Pennebaker, J. L. Mitchell, Jr. G. G. Langdon, and R. B. Arps. An overview of the basic principles of the Q-coder adaptive binary arithmetic coder. IBM Journal of Research and Development, 32(6):717-726, Nov 1988.

[37] Greg Roelofs. PNG (portable network graphics) home site. http://www.libpng. org/pub/png/.

[38] Amir Said and William A. Pearlman. A new, fast, and efficient image codec based on set partitioning in hierarchical trees. IEEE Transactions on Circuits and Systems for Video Technology, 6(3):243-250, Jun 1996.

[39] Eugene S. Schwartz and Bruce Kallick. Generating a canonical prefix encoding. Communications of the ACM, 7(3):166-169, Mar 1964.

[40] Joshua Senecal, Mark A. Duchaineau, and Kenneth I. Joy. Length-limited variableto-variable length codes for high-performance entropy coding. In James A. Storer and Martin Cohn, editors, Proceedings, Data Compression Conference (DCC 2004), pages 389-398. IEEE Computer Society, Mar 2004.

[41] Joshua Senecal, Mark A. Duchaineau, and Kenneth I. Joy. Reversible $n$-bit to $n$-bit integer Haar-like transforms. In M. H. Hamza, editor, Proceedings of the 7th IASTED Conference on Computer Graphics and Imaging, pages 135-140. ACTA Press, August 2004.

[42] Joshua Senecal, Mark A. Duchaineau, and Kenneth I. Joy. Reversible n-bit to nbit integer Haar-like transforms. In James A. Storer and Martin Cohn, editors, Proceedings, Data Compression Conference (DCC 2004), page 564. IEEE Computer Society, Mar 2004. 
[43] Joshua G. Senecal, Peter Lindstrom, Mark A. Duchaineau, and Kenneth I. Joy. An improved n-bit to n-bit reversible Haar-like transform. In Proceedings of the 12th Pacific Conference on Computer Graphics and Applications, pages 371-380. IEEE Computer Society, Oct 2004.

[44] Joshua G. Senecal, Peter Lindstrom, Mark A. Duchaineau, and Kenneth I. Joy. Investigating lossy image coding using the PLHaar transform. In James A. Storer and Martin Cohn, editors, Proceedings, Data Compression Conference (DCC 2005), page 479. IEEE Computer Society, March 2005.

[45] Claude Shannon. A mathematical theory of communication. The Bell System Technical Journal, 27:379-423, 1948.

[46] Jerome M. Shapiro. Embedded image coding using zerotrees of wavelet coefficients. IEEE Transactions on Signal Processing, 41(13):3445-3462, Dec 1993.

[47] Top 500 Supercomputer Sites. Top 500 list for November 2004. http: //www. top500. org/lists/2004/11/, 2004.

[48] Peter R. Stubley. Design and Analysis of Dual-Tree Entropy Codes. PhD thesis, University of Waterloo, 1992.

[49] Peter R. Stubley. Adaptive variable-to-variable length codes. In DCC '94: Data Compression Conference, pages 98-105, 1994.

[50] Peter R. Stubley. Personal correspondence. 2003.

[51] M. Swanson and A. Tewfik. A binary wavelet decomposition of binary images. IEEE Transactions on Information Processing, 3(12):1637-1650, Dec 1996.

[52] Michael J. Tarr. http://www.cog.brown.edu/〜 tarr/stimuli.html.

[53] CCITT Facsimile test images. ftp://ftp.funet.fi/pub/graphics/misc/ test-images/.

[54] Jeffery Scott Vitter. Design and analysis of dynamic Huffman codes. Journal of the Association for Computing Machinery, 34(4):825-845, October 1987.

[55] Jeffrey Scott Vitter. Algorithm 673: Dynamic Huffman coding. ACM Transactions on Mathematical Software, 15(2):158-167, 1989.

[56] Alan Watt and Mark Watt. Advanced Animation and Rendering Techniques, chapter 4.7.1, pages 140-145. ACM Press, 1992.

[57] Wm. Douglas Withers. The ELS-coder: A rapid entropy coder. In Proceedings DCC '97: Data Compression Conference, page 475. IEEE Computer Society, 1997.

[58] Wm. Douglas Withers. A rapid entropy-coding algorithm. Dr. Dobbs Journal, 22(4), Apr 1997.

[59] Wm. Douglas Withers. A rapid probabilty estimator and binary arithmetic coder. IEEE Transactions on Information Theory, 47(4):1533-1537, May 2001. 
[60] Wm. Douglas Withers. Personal correspondence. 2003.

[61] Ian H. Witten, Radford M. Neal, and John G. Cleary. Arithmetic coding for data compression. Communications of the ACM, 30(6):520-540, Jun 1987. 